\title{
Total Synthesis of the Photorhabdus temperata ssp. Cinereal 3240 Zwitterionic Trisaccharide Repeating Unit
}

\author{
Johny M. Nguyen* and Steven D. Townsend \\ *Department of Chemistry, Vanderbilt University, 7330 Stevenson Center, Nashville, Tennessee \\ 37235, United States \\ Email: steven.d.townsend@vanderbilt.edu
}

\section{Supporting Information}

\section{Table of Contents}

I. Title and authors

$\begin{array}{ll}\text { II. General procedure, materials, and instrumentation } & \text { S2 }\end{array}$

III. List of NMR spectra for new compounds $\quad$ S3

IV. Supplemental scheme S1. Synthetic route to building block $14 \quad$ S4

$\begin{array}{ll}\text { V. Compound characterization and preparation } & \text { S5-S28 }\end{array}$

VI. Table S1. Comparison of ${ }^{1} \mathrm{H}$ and ${ }^{13} \mathrm{C}$ NMR chemical shifts (ppm) between isolated polysaccharide and synthetic 29

VII. $\quad{ }^{1} \mathrm{H},{ }^{13} \mathrm{C}, 2 \mathrm{D}$, and ${ }^{31} \mathrm{P}$ NMR spectra of synthetic products and intermediates $\quad$ S30-S102

$\begin{array}{lll}\text { VIII. Supporting information references } & \text { S103 }\end{array}$ 
General Procedure: All non-aqueous reactions were performed in flame-dried or oven dried round-bottomed flasks under an atmosphere of nitrogen or argon, unless otherwise noted. Stainless steel syringes or cannula were used to transfer air- and moisture-sensitive liquids. Reaction temperatures were controlled using a thermocouple thermometer and analog hotplate stirrer. Reactions were conducted at room temperature (rt, approximately $23{ }^{\circ} \mathrm{C}$ ) unless otherwise noted. The anhydrous solvents used in the reactions were obtained from an MBraun MB-SPS 800 anhydrous Solvent System. Solvent for chromatography were of analytical grade and distilled under reduced pressure prior to use. Commercially available reagents were obtained from Aldrich, Fisher, TCI, TRC, and Carbosynth. Flash column chromatography was conducted as described Still et. al. using silica gel $230-400$ mesh. ${ }^{1}$ Where necessary, silica gel was neutralized by treatment of the silica gel prior to chromatography with the eluent containing $1 \%$ triethylamine. TEAB buffer was prepared by filling TEA $(7 \mathrm{~mL})$ in a measuring cylinder and adding water until the total volume reached $500 \mathrm{~mL}$. The solution was transferred to a flask and $\mathrm{CO}_{2}$ was bubbled through the solution for $2 \mathrm{~h}$ at $0{ }^{\circ} \mathrm{C}$. The buffer was stored at $4{ }^{\circ} \mathrm{C}$. Thin layer chromatography (TLC) was performed using glass backed 60-F254 silica gel plates obtained from Silicycle. Visualization of TLC plates was performed by UV (215 nm and $254 \mathrm{~nm})$, CAM stain $(5 \%(\mathrm{w} / \mathrm{v})$ ammonium molybdate, $1 \%(\mathrm{w} / \mathrm{v})$ cerium (II) sulfate and $10 \%(\mathrm{v} / \mathrm{v})$ sulfuric acid in water) or ninhydrin stain $(1.5 \%(\mathrm{w} / \mathrm{v})$ ninhydrin and $3 \%(\mathrm{v} / \mathrm{v})$ acetic acid in n-butanol) dipping solutions. Size exclusion chromatography (SEC) was performed using Bio Gel ${ }^{\circledR}$ P-2 Gel (Bio-Rad). Powdered $4 \AA$ molecular sieves were obtained from Sigma-Aldrich and used for reactions. Sieves were activated by iterative heating (to $180^{\circ} \mathrm{C}$ ) and cooling (to $25^{\circ} \mathrm{C}$ ) cycles (minimum of 3 times). Heating was carried out by microwave irradiation and cooling took place in a desiccator equipped with Drierite ${ }^{\mathrm{TM}}$ and $\mathrm{P}_{2} \mathrm{O}_{5}$.

Instrumentation: ${ }^{1} \mathrm{H}$ NMR, ${ }^{13} \mathrm{C}$ NMR, ${ }^{31} \mathrm{P}$ and $2 \mathrm{D}$ NMR spectra were recorded in the Vanderbilt Small Molecule NMR Facility on a Bruker 400 and $600 \mathrm{MHz}$. Chemical shifts are reported in parts per million (ppm) of the $\delta$ scale. Spectra were recorded in $\mathrm{CDCl}_{3}$ by using the solvent residual peak chemical shift as the internal standard $\left(\mathrm{CDCl}_{3}: \mathrm{S} 77.26 \mathrm{ppm}{ }^{1} \mathrm{H}, 77.0 \mathrm{ppm}{ }^{13} \mathrm{C}\right)$ or in $\mathrm{D}_{2} \mathrm{O}$ using the solvent as the internal standard in ${ }^{1} \mathrm{H}$ NMR $\left(\mathrm{D}_{2} \mathrm{O}: 4.79 \mathrm{ppm} 1 \mathrm{H}\right)$ unless otherwise stated. ${ }^{31} \mathrm{P}$ NMR spectra $1 \% \mathrm{H}_{3} \mathrm{PO}_{4}$ in $\mathrm{D}_{2} \mathrm{O}$ was used as an external standard. $1 \mathrm{H}$ NMR spectral data are presented as follows: Chemical shifts $(\delta \mathrm{ppm})$, multiplicity $(\mathrm{s}=$ singlet, $\mathrm{d}=$ doublet, $\mathrm{dd}=$ doublet of doublets, $\mathrm{dq}=$ doublet of quadruplet, $\mathrm{ddd}=$ doublet of doublet of doublet, $\mathrm{t}=$ triplet, $\mathrm{q}=$ quartet, $\mathrm{p}=$ pentet, $\mathrm{br}=$ broad, $\mathrm{m}=$ multiplet $)$ coupling constants $(\mathrm{Hz})$, integration. High-resolution mass spectra (HRMS) were obtained from the Department of Chemistry, Vanderbilt University using an LTQ-Orbitrap XL mass spectrometer. Optical rotations (OR) were measured with on a AUTOPOL IV digital polarimeter. Concentrations (c) in $\mathrm{g} / 100 \mathrm{~mL}$ and solvent are given in parentheses and the reported value is an average of $n=3$ independent measurements. 


\section{List of NMR spectra for new compounds}

${ }^{1} \mathrm{H},{ }^{13} \mathrm{C}$, and 2D NMR of compound $\mathbf{1 0}$

S30-S33

${ }^{1} \mathrm{H},{ }^{13} \mathrm{C}$, and 2D NMR of compound $\mathbf{1 1}$

S34-S37

${ }^{1} \mathrm{H},{ }^{13} \mathrm{C}$, and 2D NMR of compound 12

S38-S42

${ }^{1} \mathrm{H},{ }^{13} \mathrm{C}$, and 2D NMR of compound $\mathbf{1 3}$

S43-S47

${ }^{1} \mathrm{H},{ }^{13} \mathrm{C}$, and 2D NMR of compound 14

S48-S51

${ }^{1} \mathrm{H},{ }^{13} \mathrm{C}$, and 2D NMR of compound $\mathbf{1 5}$

S52-S54

${ }^{1} \mathrm{H},{ }^{13} \mathrm{C}$, and 2D NMR of compound $\mathbf{1 7}$

S55-S57

${ }^{1} \mathrm{H},{ }^{13} \mathrm{C}$, and 2D NMR of compound $\mathbf{1 8}$

S58-S60

${ }^{1} \mathrm{H},{ }^{13} \mathrm{C}$, and 2D NMR of compound 19

S61-S63

${ }^{1} \mathrm{H},{ }^{13} \mathrm{C}$, and 2D NMR of compound 20

S64-S66

${ }^{1} \mathrm{H},{ }^{13} \mathrm{C}$, and 2D NMR of compound 31

S67-S70

${ }^{1} \mathrm{H},{ }^{13} \mathrm{C}$, and 2D NMR of compound 32

S71-S75

${ }^{1} \mathrm{H},{ }^{13} \mathrm{C}$, and 2D NMR of compound 33

S76-S80

${ }^{1} \mathrm{H},{ }^{13} \mathrm{C}$, and 2D NMR of compound 34

S81-S85

${ }^{1} \mathrm{H},{ }^{13} \mathrm{C}$, and 2D NMR of compound $\mathbf{3 5}$

S86-S90

${ }^{1} \mathrm{H},{ }^{13} \mathrm{C}, 2 \mathrm{D}$ and ${ }^{31} \mathrm{P}$ NMR of compound $\mathbf{3 6}$

S91-S96

${ }^{1} \mathrm{H},{ }^{13} \mathrm{C}, 2 \mathrm{D}$ and ${ }^{31} \mathrm{P}$ NMR of compound 29

S97-S102 
Experimental protocols:

Supplemental scheme S1. Synthesis of AAT trichloroacetimidate donor 15

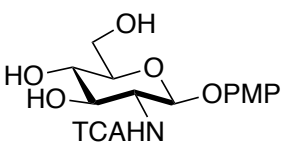

9

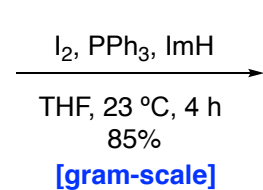

[gram-scale]

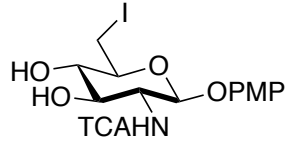

10

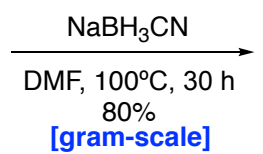

[gram-scale]

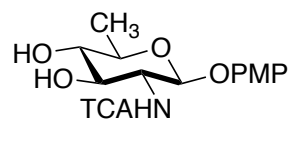

11

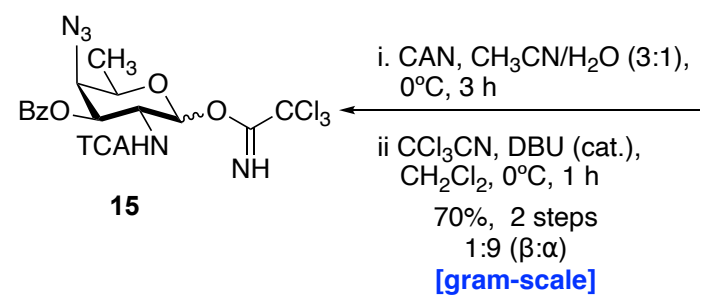

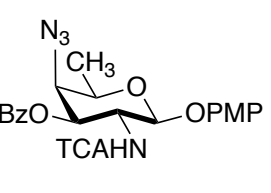

13

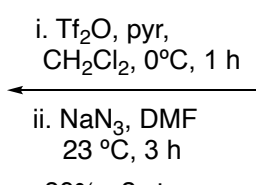

$80 \%, 2$ steps

[gram-scale]

Proton and Carbon assignment based on the following figure:

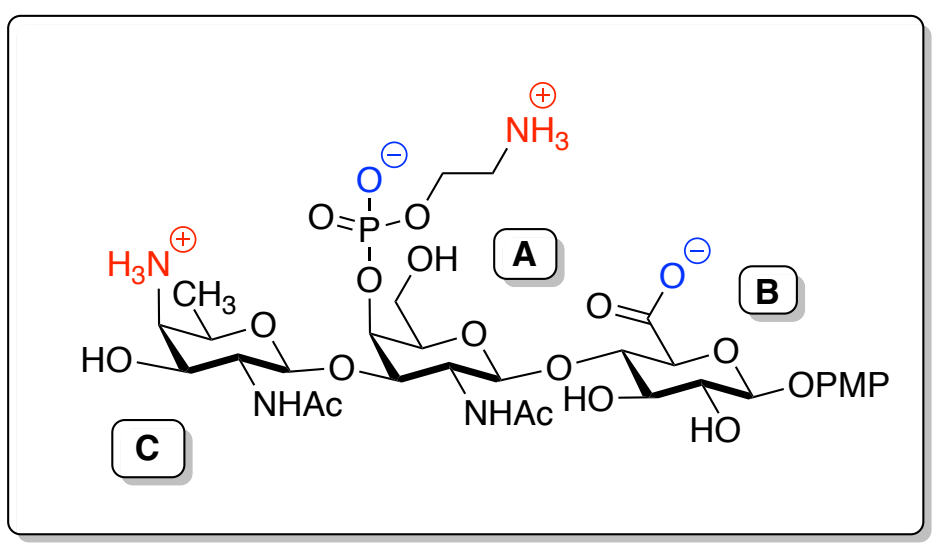




\section{p-Methoxyphenyl 2,6-dideoxy-2-N-trichloroacetamido- $\beta$-D-glucopyranoside (10):}

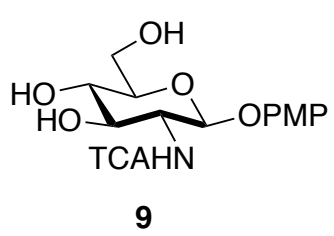

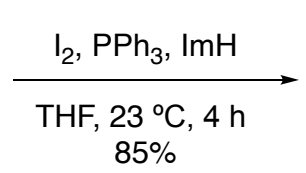

$85 \%$

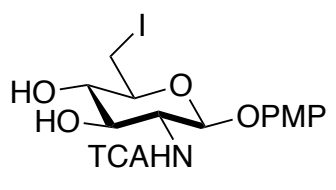

10

To a solution of $\mathrm{PPh}_{3}$ (4.48 g, 17.1 mmol, 1.0 equiv.), imidazole (2.77 g, 40.6 mmol, 2.5 equiv., and $\mathrm{I}_{2}$ (4.33 g, $17.1 \mathrm{mmol}, 1.0$ equiv.) in anhydrous THF (81 mL) under an argon atmosphere was added triol $9^{2}$ (7.00 g, $16.3 \mathrm{mmol}, 1.0$ equiv.). The mixture stirred at room temperature for $4 \mathrm{~h}$. At completion, the reaction mixture was quenched by the addition of satd. aq. $\mathrm{Na}_{2} \mathrm{~S}_{2} \mathrm{O}_{3}(25 \mathrm{~mL} \times 1)$ and extracted with $\mathrm{CH}_{2} \mathrm{Cl}_{2}(3 \times 50 \mathrm{~mL})$. The combined organic layers were dried over anhydrous $\mathrm{Na}_{2} \mathrm{SO}_{4}$, filtered, and concentrated in vacuo. Flash chromatography of the crude material eluting with EtOAc/Hexane (6:4) gave 6-iodo derivative 10 (7.5 g, $16.3 \mathrm{mmol}, 85 \%)$ as a white foam. 6iodo derivative $\mathbf{1 0}$ had $\mathrm{R}_{\mathrm{f}} 0.42$ (EtOAc/Hexane 7:3) visualized with ceric ammonium molybdate stain; $[\alpha] \mathrm{D}^{25}=-3.9^{\circ}\left(\mathrm{c}=0.01, \mathrm{CH}_{3} \mathrm{OH}\right) ;{ }^{1} \mathrm{H}$ NMR $(600 \mathrm{MHz}, \mathrm{MeOD}) \delta 7.08(\mathrm{~d}, J=9.1 \mathrm{~Hz}, 2 \mathrm{H}$, Ar), $6.81(\mathrm{~d}, J=9.1 \mathrm{~Hz}, 2 \mathrm{H}, \mathrm{Ar}), 5.02$ (d, $J=8.4 \mathrm{~Hz}, 1 \mathrm{H}, \mathrm{H}-1), 3.93$ (dd, $J=10.5,8.5 \mathrm{~Hz}, 1 \mathrm{H}, \mathrm{H}-$ 2), 3.74 (s, 3H, $\left.\mathrm{OCH}_{3}\right), 3.72-3.65$ (m, 2H, H-3, H-6), 3.37 - 3.26 (m, 3H, H-4, H-5, H-6); ${ }^{13} \mathrm{C}$ NMR (151 MHz, MeOD) $\delta 163.2(\mathrm{NHCO}), 155.5,151.7,118.4,114.0,100.4(\mathrm{C}-1), 92.7\left(\mathrm{CCl}_{3}\right)$, 76.1 (C-5), 74.6 (C-4), 73.1 (C-3), 57.8 (C-2), 54.7 (OCH3), 4.3 (C-6); HRMS (ESI) calcd. for $\mathrm{C}_{15} \mathrm{H}_{16} \mathrm{O}_{6} \mathrm{Cl}_{3} \mathrm{IN}(\mathrm{M}-\mathrm{H})^{-}$537.9093, found $537.9083 \mathrm{~m} / \mathrm{z}$. 


\section{p-Methoxyphenyl 2,6-dideoxy-6-iodo-2-trichloroacetamido- $\beta$-D-glucopyranoside (11):}

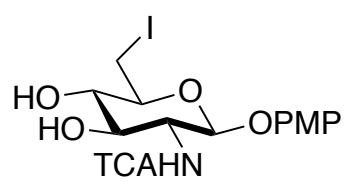

10

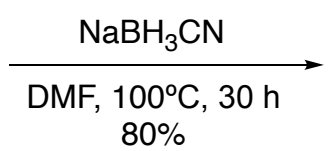

$80 \%$

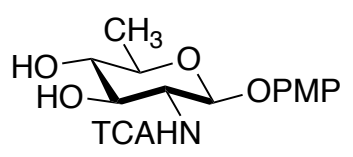

11

A solution of 6-iodo derivative 10 (10.0 g, $18.5 \mathrm{mmol}, 1.0$ equiv.) was dissolved in anhydrous DMF $(92 \mathrm{~mL})$ under an argon atmosphere. Sodium cyanoborohydride $(7.81 \mathrm{~g}, 211 \mathrm{mmol}, 5.0$ equiv) was added and the reaction mixture was stirred at $100{ }^{\circ} \mathrm{C}$ for $30 \mathrm{~h}$. At completion, the reaction mixture was diluted with $\mathrm{H}_{2} \mathrm{O}(300 \mathrm{~mL})$ and extracted with $\mathrm{CH}_{2} \mathrm{Cl}_{2}(3 \times 100 \mathrm{~mL})$. The combined organic layers were dried over $\mathrm{Na}_{2} \mathrm{SO}_{4}$, filtered, and concentrated in vacuo. Flash chromatography of the crude material eluting with $\mathrm{CH}_{2} \mathrm{Cl}_{2} / \mathrm{CH}_{3} \mathrm{OH}$ (95:5) gave diol 10 (5.34 g, $12.9 \mathrm{mmol}, 70 \%)$ as a white foam. Diol 11 had $\mathrm{R}_{\mathrm{f}} 0.33\left(\mathrm{CH}_{2} \mathrm{Cl}_{2} / \mathrm{CH}_{3} \mathrm{OH} 19: 1\right)$ visualized with ceric ammonium molybdate stain; $[\alpha] \mathrm{D}^{25}=-12.5^{\circ}\left(\mathrm{c}=0.014, \mathrm{CH}_{3} \mathrm{OH}\right) ;{ }^{1} \mathrm{H} \mathrm{NMR}(600 \mathrm{MHz}, \mathrm{MeOD}) \delta$ $6.94(\mathrm{~d}, J=9.1 \mathrm{~Hz}, 2 \mathrm{H}, \mathrm{Ar}), 6.81$ (d, $J=9.1 \mathrm{~Hz}, 2 \mathrm{H}, \mathrm{Ar}), 5.01$ (d, $J=8.5 \mathrm{~Hz}, 1 \mathrm{H}, \mathrm{H}-1), 3.90$ (dd, $J=10.5,8.5 \mathrm{~Hz}, 1 \mathrm{H}, \mathrm{H}-2), 3.74\left(\mathrm{~s}, 3 \mathrm{H}, \mathrm{OCH}_{3}\right), 3.65(\mathrm{dd}, J=10.5,8.8 \mathrm{~Hz}, 1 \mathrm{H}, \mathrm{H}-3), 3.46(\mathrm{dq}, J=$ 9.4, $6.1 \mathrm{~Hz}, 1 \mathrm{H}, \mathrm{H}-5), 3.14$ (t, $J=9.1 \mathrm{~Hz}, 1 \mathrm{H}, \mathrm{H}-4), 1.35$ (d, $J=6.1 \mathrm{~Hz}, 3 \mathrm{H}, \mathrm{H}-6) ;{ }^{13} \mathrm{C}$ NMR $(151$ $\mathrm{MHz}, \mathrm{MeOD}) \delta 163.2(\mathrm{NHCO}), 155.4,151.7,117.9,114.0,100.1$ (C-1), $92.8\left(\mathrm{CCl}_{3}\right), 76.1$ (C-4), 73.3 (C-3), 72.0 (C-5), 58.0 (C-2), $54.6\left(\mathrm{OCH}_{3}\right), 16.7$ (C-6); HRMS (ESI) calcd. for $\mathrm{C}_{15} \mathrm{H}_{17} \mathrm{O}_{6} \mathrm{Cl}_{3} \mathrm{~N}$ $(\mathrm{M}-\mathrm{H})^{-}$412.0127, found $412.0127 \mathrm{~m} / \mathrm{z}$. 
p-Methoxyphenyl 3-O-benzoyl-2,6-dideoxy-2-trichloroacetamido- $\beta$-D-glucopyranoside (12):

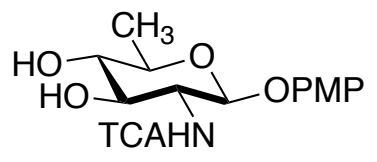

11

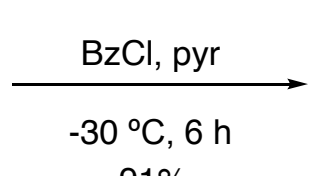

$91 \%$

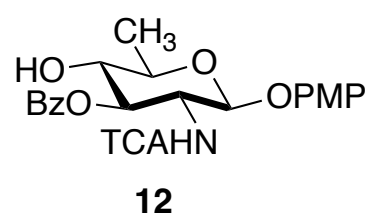

12

A solution of diol 11 (2.91 g, $6.91 \mathrm{mmol}, 1.0$ equiv.) was dissolved in anhydrous pyridine $(70 \mathrm{~mL})$ under an argon atmosphere and cooled to $-30^{\circ} \mathrm{C}$. A diluted solution of benzoyl chloride $(0.82 \mathrm{~mL}$, $6.9 \mathrm{mmol}, 1.0$ equiv.) in $1.0 \mathrm{~mL}$ pyridine was then added dropwise over $10 \mathrm{~min}$. The reaction mixture was held at $-30^{\circ} \mathrm{C}$ for $6 \mathrm{~h}$. At completion, the solution was diluted with EtOAc $(100 \mathrm{~mL})$ and washed with $2 \mathrm{M} \mathrm{HCl}(100 \mathrm{~mL} \times 5)$, sat. aq. $\mathrm{NaHCO}_{3}(100 \mathrm{~mL} \times 1)$, and brine $(100 \mathrm{~mL} \times 1)$. The combined organic layers were dried over anhydrous $\mathrm{Na}_{2} \mathrm{SO}_{4}$, filtered, and concentrated in vacuo. Flash chromatography of the crude material eluting with EtOAc/Hexane (1:5) gave the 3O-benzoyl derivative $12(3.31 \mathrm{~g}, 6.41 \mathrm{mmol}, 91 \%)$ as a white foam. Alcohol 12 had $\mathrm{R}_{\mathrm{f}} 0.60$ (EtOAc/Hexane 3:1) visualized with ceric ammonium molybdate stain; $[\alpha] \mathrm{D}^{25}=+9.7^{\circ}(\mathrm{c}=$ 0.0019, $\left.\mathrm{CHCl}_{3}\right) ;{ }^{1} \mathrm{H} \mathrm{NMR}\left(600 \mathrm{MHz}, \mathrm{CDCl}_{3}\right) \delta 7.97(\mathrm{dd}, J=8.4,1.4 \mathrm{~Hz}, 2 \mathrm{H}, \mathrm{Ar}), 7.61-7.55(\mathrm{~m}$, 1H, Ar), $7.41(\mathrm{dd}, J=8.3,7.4 \mathrm{~Hz}, 2 \mathrm{H}, \mathrm{Ar}), 7.27(\mathrm{~d}, J=9.5 \mathrm{~Hz}, 1 \mathrm{H}, \mathrm{NH}), 6.96(\mathrm{~d}, J=9.1 \mathrm{~Hz}, 2 \mathrm{H}$, Ar), $6.79(\mathrm{~d}, J=9.1 \mathrm{~Hz}, 2 \mathrm{H}, \mathrm{Ar}), 5.63(\mathrm{dd}, J=10.8,8.3 \mathrm{~Hz}, 1 \mathrm{H}, \mathrm{H}-3), 5.13$ (d, $J=8.4 \mathrm{~Hz}, 1 \mathrm{H}, \mathrm{H}-$ 1), 4.40 (ddd, $J=10.8,9.1,8.3 \mathrm{~Hz}, 1 \mathrm{H}, \mathrm{H}-2), 3.76$ (s, 3H, OCH $), 3.73$ - 3.56 (m, 2H, H-4, H-5), $1.44(\mathrm{~d}, J=5.6 \mathrm{~Hz}, 3 \mathrm{H}, \mathrm{H}-6) ;{ }^{13} \mathrm{C}$ NMR (151 MHz, $\left.\mathrm{CDCl}_{3}\right) \delta 167.9(C \mathrm{OPh}), 162.5(\mathrm{NHCO})$, 155.8, 151.5, 134.0, 130.0, 128.8, 128.7, 118.9, 114.7, $100.5(\mathrm{C}-1), 92.3\left(\mathrm{CCl}_{3}\right), 75.7(\mathrm{C}-3), 74.5$ (C-4), 72.4 (C-5), 56.3 (C-2), $55.7\left(\mathrm{OCH}_{3}\right), 17.8$ (C-6); HRMS (ESI) calcd. for $\mathrm{C}_{22} \mathrm{H}_{21} \mathrm{O}_{7} \mathrm{Cl}_{3} \mathrm{~N}(\mathrm{M}-$ H) 516.0389 , found $516.0378 \mathrm{~m} / \mathrm{z}$. 


\section{$p$-Methoxyphenyl 3- $O$-benzoyl-4-azido-2,4,6-trideoxy-2-trichloroacetamido- $\beta$-D- galactopyranoside (13):}

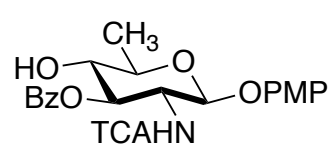

12

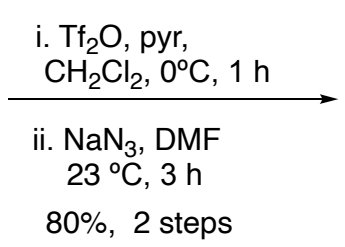

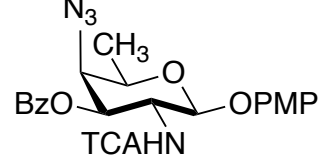

13

Alcohol 12 (1.80 g, 3.50 mmol, 1.0 equiv.) was dissolved in anhydrous $\mathrm{CH}_{2} \mathrm{Cl}_{2}(35 \mathrm{~mL})$ under an argon atmosphere. The reaction mixture was cooled to $0{ }^{\circ} \mathrm{C}$ and anhydrous pyridine $(0.56 \mathrm{~mL}, 6.9$ mmol, 2.0 equiv.) was added followed by triflic anhydride (0.70 mL, $4.2 \mathrm{mmol}, 1.2$ equiv. $)$. After stirring for $1 \mathrm{~h}$ at $0{ }^{\circ} \mathrm{C}$, the reaction mixture was diluted with $\mathrm{CH}_{2} \mathrm{Cl}_{2}(100 \mathrm{~mL})$ and washed with $\mathrm{H}_{2} \mathrm{O}(100 \mathrm{~mL} \times 1)$ and brine $(100 \mathrm{~mL} \times 1)$. The organic layer was dried over anhydrous $\mathrm{Na}_{2} \mathrm{SO}_{4}$, filtered and concentrated in vacuo. The residue was dissolved in anhydrous DMF (30 mL) and $\mathrm{NaN}_{3}$ (599 mg, $9.22 \mathrm{mmol}, 3.0$ equiv.) was added. After stirring for $3 \mathrm{~h}$ at $\mathrm{rt}$, the reaction mixture was diluted with EtOAc $(150 \mathrm{~mL})$ and washed with $\mathrm{H}_{2} \mathrm{O}(100 \mathrm{~mL} \times 1)$ and brine $(100 \mathrm{~mL} \times 1)$. The organic phase was dried over anhydrous $\mathrm{Na}_{2} \mathrm{SO}_{4}$, filtered, and concentrated in vacuo. Flash chromatography of the crude material eluting with EtOAc/Hexane (1:4) gave the azido derivative $13(1.30 \mathrm{~g}, 2.40 \mathrm{mmol}, 78 \%)$ as a white solid. The trideoxy intermediate 13 had $\mathrm{R}_{\mathrm{f}}=0.50$ $\left(\right.$ EtOAc/Hexane 2:3) visualized with ceric ammonium molybdate stain; $[\alpha] \mathrm{D}^{25}=-23.3^{\circ}(\mathrm{c}=0.015$, $\left.\mathrm{CHCl}_{3}\right) ;{ }^{1} \mathrm{H} \mathrm{NMR}\left(600 \mathrm{MHz}, \mathrm{CDCl}_{3}\right) \delta 8.08(\mathrm{dd}, J=8.3,1.3 \mathrm{~Hz}, 2 \mathrm{H}, \mathrm{Ar}), 7.63-7.59(\mathrm{~m}, 1 \mathrm{H}$, Ar), $7.47(\mathrm{dd}, J=8.3,7.4 \mathrm{~Hz}, 2 \mathrm{H}, \mathrm{Ar}), 6.97$ (d, $J=9.0 \mathrm{~Hz}, 2 \mathrm{H}, \mathrm{Ar}), 6.88(\mathrm{~d}, J=8.6 \mathrm{~Hz}, 1 \mathrm{H}, \mathrm{NH})$, $6.79(\mathrm{~d}, J=9.0 \mathrm{~Hz}, 2 \mathrm{H}, \mathrm{Ar}), 5.77$ (dd, $J=11.2,3.6 \mathrm{~Hz}, 1 \mathrm{H}, \mathrm{H}-3), 5.15$ (d, $J=8.3 \mathrm{~Hz}, 1 \mathrm{H}, \mathrm{H}-1)$, $4.48(\mathrm{dt}, J=11.2,8.5 \mathrm{~Hz}, 1 \mathrm{H}, \mathrm{H}-2), 4.03(\mathrm{dd}, J=3.6,1.3 \mathrm{~Hz}, 1 \mathrm{H}, \mathrm{H}-4), 3.96$ (qd, $J=6.3,1.4 \mathrm{~Hz}$, 1H, H-5), 3.76 (s, 3H, OCH $), 1.45(\mathrm{~d}, J=6.3 \mathrm{~Hz}, 3 \mathrm{H}, \mathrm{H}-6) ;{ }^{13} \mathrm{C} \mathrm{NMR}\left(151 \mathrm{MHz}, \mathrm{CDCl}_{3}\right) \delta 166.1$ (COPh), 162.2 (NHCO), 155.8, 151.1, 134.0, 130.1, 128.6, 128.3, 119.1, 114.5, 100.4 (C-1), 92.2 
$\left(\mathrm{CCl}_{3}\right), 72.0$ (C-3), 69.6 (C-5), 63.5 (C-4), $55.6(\mathrm{C}-5), 53.3\left(\mathrm{OCH}_{3}\right), 17.5$ (C-6); HRMS (ESI) calcd. for $\mathrm{C}_{22} \mathrm{H}_{20} \mathrm{O}_{6} \mathrm{Cl}_{3} \mathrm{~N}_{4}(\mathrm{M}-\mathrm{H})^{-} 541.0454$, found $541.0442 \mathrm{~m} / z$.

p-Methoxyphenyl 4-azido-2,4,6-trideoxy-2-trichloroacetamido- $\beta$-D- galactopyranoside (14):

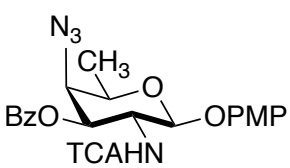

13

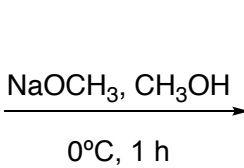

$96 \%$

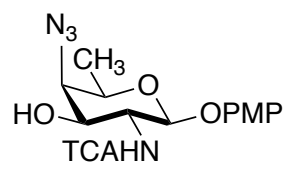

14

A solution of 13 (125 mg, $0.21 \mathrm{mmol}, 1.0$ equiv.) in anhydrous $\mathrm{CH}_{3} \mathrm{OH}(4 \mathrm{~mL})$ under an argon atmosphere was added $\mathrm{NaOCH}_{3}$ solution $\left(0.10 \mathrm{~mL}, 1.5 \mathrm{mmol},[1.5 \mathrm{M}]\right.$ in $\left.\mathrm{CH}_{3} \mathrm{OH}\right)$ dropwise at room temperature. At $1 \mathrm{~h}$, the $\mathrm{pH}$ of the reaction mixture was still $>12$ and TLC indicated that the starting material had been completely consumed for a more polar spot. The reaction was neutralized by the addition of acidic form Amberlite IR $120(\mathrm{H}+)$ ion exchange resin. The solution was filtered through a glass fritted funnel with a pad of Celite to remove the resin. The filtrate was concentrated in vacuo to give $14(85.0 \mathrm{mg}, 0.19 \mathrm{mmol},>95 \%)$ as a white foam in quantitative yield. $\mathrm{Rf}=0.40\left(\mathrm{EtOAc} /\right.$ Hexanes 1:1) visualized with ceric ammonium molybdate stain; $[\alpha] \mathrm{D}^{25}=$ $-28.9^{\circ}\left(\mathrm{c}=0.016, \mathrm{CHCl}_{3}\right) ;{ }^{1} \mathrm{H} \mathrm{NMR}\left(600 \mathrm{MHz}, \mathrm{CDCl}_{3}\right) \delta 7.02(\mathrm{~d}, J=6.4 \mathrm{~Hz}, 1 \mathrm{H}, \mathrm{NH}), 6.97(\mathrm{~d}$, $J=9.1 \mathrm{~Hz}, 1 \mathrm{H}, \mathrm{Ar}), 6.80(\mathrm{~d}, J=9.1 \mathrm{~Hz}, 1 \mathrm{H}, \mathrm{Ar}), 5.05(\mathrm{~d}, J=8.3 \mathrm{~Hz}, 1 \mathrm{H}, \mathrm{H}-1), 4.45$ (ddd, $J=$ 10.5, 6.6, 3.7 Hz, 1H, H-3), $3.89-3.83$ (m, 1H, H-2), 3.83-3.79 (m, 2H, H-4, H-5), 3.76 (s, 3H, $\left.\mathrm{OCH}_{3}\right), 3.15(\mathrm{~d}, J=6.6 \mathrm{~Hz}, 1 \mathrm{H}, \mathrm{OH}), 1.43(\mathrm{~d}, J=6.3 \mathrm{~Hz}, 3 \mathrm{H}, \mathrm{H}-6) ;{ }^{13} \mathrm{C} \mathrm{NMR}\left(151 \mathrm{MHz}, \mathrm{CDCl}_{3}\right)$ $\delta 163.0(\mathrm{NHCO}), 155.8,150.8,119.1,114.6,99.5(\mathrm{C}-1), 92.2\left(\mathrm{CCl}_{3}\right), 70.6(\mathrm{C}-3), 70.0(\mathrm{C}-5), 65.7$ (C-4), $57.0(\mathrm{C}-2), 55.6\left(\mathrm{OCH}_{3}\right), 17.5(\mathrm{C}-6)$; HRMS (ESI) calcd. for $\mathrm{C}_{15} \mathrm{H}_{17} \mathrm{O}_{5} \mathrm{Cl}_{3} \mathrm{~N}_{4} \mathrm{Na}(\mathrm{M}+\mathrm{Na})^{+}$ 461.0164, found $461.0157 \mathrm{~m} / \mathrm{z}$. 


\section{3-O-benzoyl-4-azido-2,4,6-trideoxy-2-trichloroacetamido- $\beta$-D- galactopyranosyl $\mathrm{N}$ - trichloroacetimidate (15):}

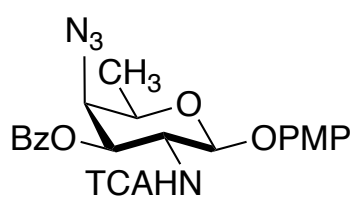

13

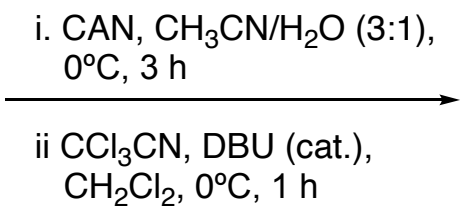

$70 \%, 2$ steps $1: 9(\beta: \alpha)$

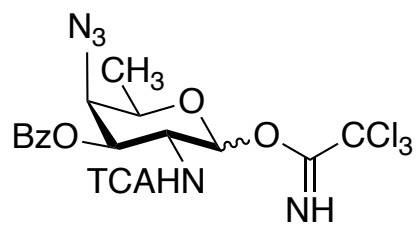

15

To a solution of compound 13 (1.10 g, $2.0 \mathrm{mmol}, 1.0$ equiv.) in $\mathrm{CH}_{3} \mathrm{CN} / \mathrm{H}_{2} \mathrm{O}(3: 1 \mathrm{v} / \mathrm{v}, 20 \mathrm{~mL})$ at $0{ }^{\circ} \mathrm{C}$ under an argon atmosphere was added cerium ammonium nitrate $(5.54 \mathrm{~g}, 10.1 \mathrm{mmol}, 5.0$ equiv.). ${ }^{3}$ The reaction mixture was stirred for $3 \mathrm{~h}$ at $0{ }^{\circ} \mathrm{C}$, the reaction mixture was diluted with EtOAc $(100 \mathrm{~mL})$ and washed with satd. aq. $\mathrm{NaHCO}_{3}(30 \mathrm{~mL} \times 1)$ and $\mathrm{H}_{2} \mathrm{O}(30 \mathrm{~mL} \times 1)$. The organic layer was dried over anhydrous $\mathrm{Na}_{2} \mathrm{SO}_{4}$, filtered and concentrated in vacuo. Flash chromatography of the crude material eluting with EtOAc/Hexane $(2: 1)$ as eluent to afford the hemiacetal as a yellow-orange foam. The compound was dissolved in $\mathrm{CH}_{2} \mathrm{Cl}_{2}(50 \mathrm{~mL})$, trichloroacetonitrile (1.0 mL, $10.3 \mathrm{mmol}, 5$ equiv.) and DBU (50 $\mu \mathrm{L}, 0.61 \mathrm{mmol}, 0.30$ equiv.) were added, and the reaction mixture was stirred at $0{ }^{\circ} \mathrm{C}$ for $1 \mathrm{~h}$. The solvent was removed under reduced pressure, and the residue was purified by flash column with EtOAc/hexane (1:1) to yield the trichloroacetimidate $\mathbf{1 5}$ as a white foam $(1.10 \mathrm{~g}, 1.9 \mathrm{mmol}, 70 \%)$. The trichloroacetimidate $\mathbf{1 5}$ had $\mathrm{R}_{\mathrm{f}}=0.80\left(\right.$ EtOAc/Hexane 1:1) visualized with ceric ammonium molybdate stain; $[\alpha] \mathrm{D}^{25}=+7.6^{\circ}$ $\left(\mathrm{c}=0.006, \mathrm{CHCl}_{3}\right) ;{ }^{1} \mathrm{H} \mathrm{NMR}\left(600 \mathrm{MHz}, \mathrm{CDCl}_{3}\right) \delta 8.79\left(\mathrm{~s}, 1 \mathrm{H}, \mathrm{OC}(\mathrm{N}) H C \mathrm{Cl}_{3}\right), 8.08(\mathrm{dd}, J=8.4$, $1.1 \mathrm{~Hz}, 2 \mathrm{H}, \mathrm{Ar}), 7.64-7.56(\mathrm{~m}, 1 \mathrm{H}, \mathrm{Ar}), 7.47(\mathrm{dd}, J=8.3,7.4 \mathrm{~Hz}, 1 \mathrm{H}, \mathrm{Ar}), 7.01(\mathrm{~d}, J=9.0 \mathrm{~Hz}$, 1H, NH), 6.47 (d, $J=3.6 \mathrm{~Hz}, 1 \mathrm{H}, \mathrm{H}-1), 5.75$ (dd, $J=11.2,3.3 \mathrm{~Hz}, 1 \mathrm{H}, \mathrm{H}-3), 4.91$ (ddd, $J=11.1$, 9.0, 3.6 Hz, 1H, H-2), 4.35 (qd, $J=6.4,1.5 \mathrm{~Hz}, 1 \mathrm{H}, \mathrm{H}-5), 4.09$ (dd, J=3.5, $1.5 \mathrm{~Hz}, 1 \mathrm{H}, \mathrm{H}-4), 1.40$ $(\mathrm{d}, J=6.4 \mathrm{~Hz}, 3 \mathrm{H}, \mathrm{C}-6) ;{ }^{13} \mathrm{C} \mathrm{NMR}\left(151 \mathrm{MHz}, \mathrm{CDCl}_{3}\right) \delta 166.9(\mathrm{COPh}), 162.2(\mathrm{NHCO}), 160.2$ $\left(\mathrm{OC}(\mathrm{NH}) \mathrm{CCl}_{3}\right), 134.2,130.3,128.8,128.2,94.9(\mathrm{C}-1), 91.9\left(\mathrm{CCl}_{3}\right), 90.9\left(\mathrm{CCl}_{3}\right), 71.0(\mathrm{C}-3), 68.2$ 
(C-5), 63.8 (C-4), 50.0 (C-2), 17.4 (C-6); HRMS (ESI) calcd. for $\mathrm{C}_{17} \mathrm{H}_{15} \mathrm{O}_{6} \mathrm{Cl}_{6} \mathrm{~N}_{5} \mathrm{Na}(\mathrm{M}+\mathrm{Na})^{+}$ 601.9104, found $601.9100 \mathrm{~m} / \mathrm{z}$.

p-Methoxyphenyl benzyl 2,3-di-O-benzoyl- $\beta$-D-glucopyranosidurinate (17):
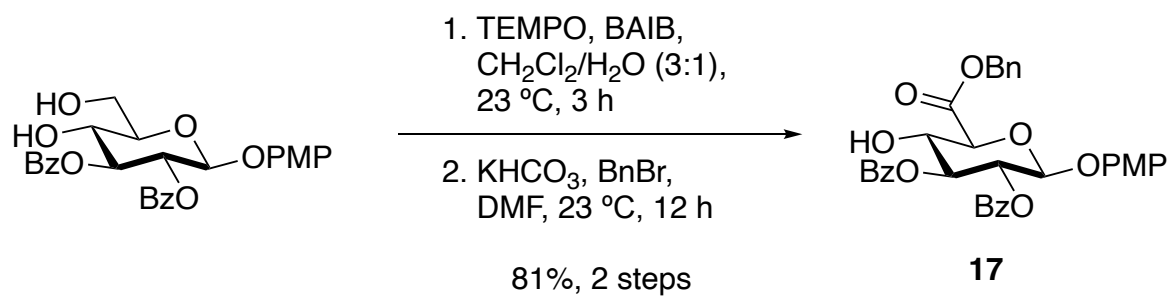

17

To a solution of known $\operatorname{diol}^{4}$ (1.00 g, $1.97 \mathrm{mmol}, 1.0$ equiv.) in $\mathrm{CH}_{2} \mathrm{Cl}_{2} / \mathrm{H}_{2} \mathrm{O}(3 / 1 \mathrm{v} / \mathrm{v}, 100 \mathrm{~mL})$ under an argon atmosphere was added 2,2,6,6-tetramethyl-1-piperidinyloxy (TEMPO) (92.2 mg, 0.59 mmol, 0.3 equiv.) and (Diacetoxyiodo) benzene (BAIB) (1.37, $4.92 \mathrm{mmol}, 2.5$ equiv.). The biphasic mixture was stirred vigorously for $3 \mathrm{~h}$ and quenched by the addition of satd. aq. $\mathrm{Na}_{2} \mathrm{~S}_{2} \mathrm{O}_{3}$ $(20 \mathrm{~mL})$. The layers were separated, and the aqueous layer was acidified with $1 \mathrm{M}$ aqueous $\mathrm{HCl}$ to pH 2 and extracted with $\mathrm{CH}_{2} \mathrm{Cl}_{2}(50 \mathrm{~mL} \times 3$. The combined organic layers were dried over anhydrous $\mathrm{Na}_{2} \mathrm{SO}_{4}$, filtered, and concentrated in vacuo. The residue was dissolved in $65.0 \mathrm{~mL}$ of anhydrous DMF. To this solution was added sequentially $\mathrm{KHCO}_{3}(1.00 \mathrm{~g}, 9.83$ mmol, 5.0 equiv.) and $\mathrm{BnBr}(0.25 \mathrm{~mL}, 2.16 \mathrm{mmol}, 1.1$ equiv. $)$ at $0{ }^{\circ} \mathrm{C}$ and the solution was stirred at $25^{\circ} \mathrm{C}$ for about $12 \mathrm{~h}$. The solvents were concentrated in vacuo, and the residue was purified by flash silica gel chromatography (50:50 EtOAc/Hexanes) to provide the glucuronic acid benzyl ester 31 (0.95 g, $1.60 \mathrm{mmol}, 81 \%$ yield) as a white amorphous solid. Glucuronic acid benzyl ester had $\mathrm{R}_{\mathrm{f}} 0.44$ (EtOAc/Hexane 1:1) visualized with ceric ammonium molybdate stain; $[\alpha] \mathrm{D}^{25}=+11.7^{\circ}(\mathrm{c}=0.011$, $\left.\mathrm{CHCl}_{3}\right) ;{ }^{1} \mathrm{H} \mathrm{NMR}\left(600 \mathrm{MHz}, \mathrm{CDCl}_{3}\right) \delta 8.00(\mathrm{dd}, J=8.4,1.3 \mathrm{~Hz}, 1 \mathrm{H}, \mathrm{Ar}), 7.97(\mathrm{dd}, J=8.4,1.4$ Hz, 1H, Ar), $7.55-7.48$ (m, 2H, Ar), $7.43-7.34$ (m, 9H, Ar), 6.95 (d, J=9.0 Hz, 2H, Ar), 6.74 (d, $J=9.1 \mathrm{~Hz}, 2 \mathrm{H}, \mathrm{Ar}), 5.67(\mathrm{dd}, J=9.6,7.6 \mathrm{~Hz}, 1 \mathrm{H}, \mathrm{H}-2), 5.60$ (t, $J=9.4 \mathrm{~Hz}, 1 \mathrm{H}, \mathrm{H}-3), 5.39-$ 
$5.23\left(\mathrm{~m}, 2 \mathrm{H}, \mathrm{PhCH}_{2}\right), 5.17$ (d, $\left.J=7.6 \mathrm{~Hz}, 1 \mathrm{H}, \mathrm{H}-1\right), 4.33$ (t, $\left.J=9.4 \mathrm{~Hz}, 1 \mathrm{H}, \mathrm{H}-4\right), 4.20$ (d, $J=9.6$

$\mathrm{Hz}, 1 \mathrm{H}, \mathrm{H}-5), 3.74\left(\mathrm{~s}, 3 \mathrm{H}, \mathrm{OCH}_{3}\right) ;{ }^{13} \mathrm{C} \mathrm{NMR}\left(151 \mathrm{MHz}, \mathrm{CDCl}_{3}\right) \delta 168.2\left(\mathrm{CO}_{2} \mathrm{Bn}\right), 166.6(\mathrm{COPh})$, $165.1(\mathrm{COPh}), 155.9,150.9,134.8,133.5,133.3,130.0,129.8,129.1,128.9,128.7,128.6,128.4$, 128.1, 119.3, 114.5, 101.2 (C-1), 74.8 (C-3), 74.6 (C-5), 71.2 (C-2), $70.3(\mathrm{C}-4), 67.7\left(\mathrm{PhCH}_{2}\right)$, $55.6\left(\mathrm{OCH}_{3}\right)$; HRMS (ESI) calcd. for $\mathrm{C}_{30} \mathrm{H}_{30} \mathrm{O}_{10} \mathrm{Na}(\mathrm{M}+\mathrm{Na})^{+} 621.1731$, found $621.1732 \mathrm{~m} / \mathrm{z}$.

p-Methoxyphenyl (4,6-O-benzylidene-3-O-tert. Butyldimethylsiyl-2-deoxy-2trichloroacetamido- $\alpha$-D- galactopyranosyl)- $(1 \rightarrow 4)-($ methyl $2,3-d i-O$-benzyl- $\beta$-Dglucopyranosid)uronate (18):

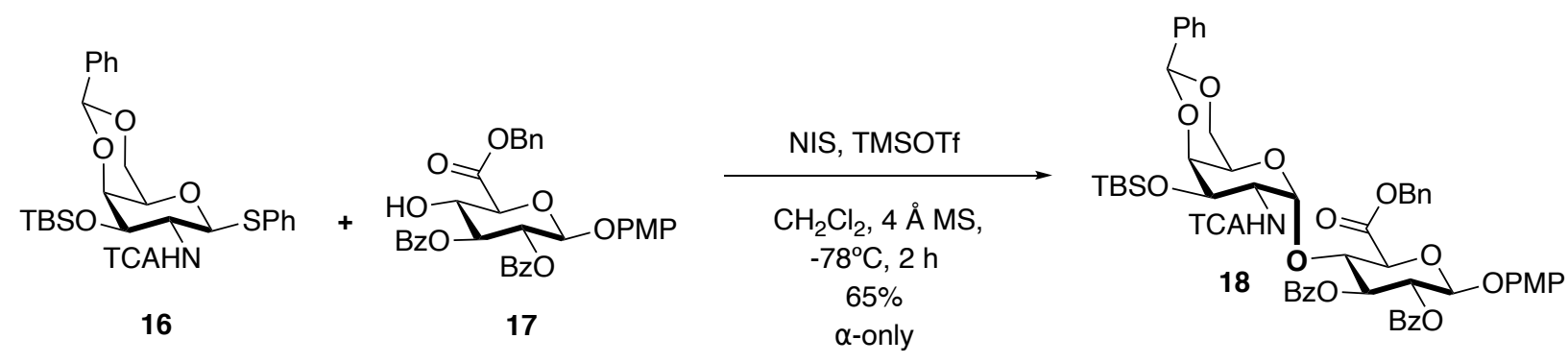

A solution of known acceptor $\mathbf{1 7}^{5}\left(250 \mathrm{mg}, 0.42 \mathrm{mmol}, 1.0\right.$ equiv.) and donor $1 \mathbf{1 6}^{6}(284 \mathrm{mg}, 0.46$ mmol, 1.1 equiv.) in anhydrous $\mathrm{CH}_{2} \mathrm{Cl}_{2}(5 \mathrm{~mL})$ under an argon atmosphere was added $4 \AA \mathrm{MS}$ $(0.5 \mathrm{~g})$ at room temperature and allowed to stir for $1 \mathrm{~h}$. The reaction mixture was cooled to $-78^{\circ} \mathrm{C}$ and added NIS (188 mg, 0.83 mmol, 2.0 equiv.). After $1 \mathrm{~min}$, a solution of TMSOTf ( 0.15 equiv., $2.0 \mathrm{~mL}, 5 \%$ in anhydrous $\mathrm{CH}_{2} \mathrm{Cl}_{2}$ ) was added dropwise. The reaction was stirred at $-78{ }^{\circ} \mathrm{C}$ for $2 \mathrm{~h}$ when TLC indicated complete consumption of the alcohol 17 starting material. The reaction was quench by the addition of dilute $\mathrm{Et}_{3} \mathrm{~N}$ in $\mathrm{CH}_{2} \mathrm{Cl}_{2}$ while warming to room temperature. The reaction was filtered through a glass fritted filter funnel equipped with a pad of Celite to remove the $4 \AA$ MS and then concentrated in vacuo. The crude oil was then diluted with EtOAc and transferred to a separatory funnel and washed with satd. aq. $\mathrm{Na}_{2} \mathrm{~S}_{2} \mathrm{O}_{3}(20 \mathrm{~mL}$ x 1), brine (20 mL x 1), and dried over anhydrous $\mathrm{Na}_{2} \mathrm{SO}_{4}$. The mixture was filtered and concentrated to a crude oil. Flash chromatography of the crude material eluting with EtOAc/Hexane (2:3) gave $\alpha$ product: (300 mg, 
$0.27 \mathrm{mmol}, 65 \%)$ as a yellow foam. $\mathrm{R}_{\mathrm{f}}=0.55($ EtOAc/Hexane $2: 3)$ visualized with ceric ammonium molybdate stain; $[\alpha] \mathrm{D}^{25}=+37.5^{\circ}\left(\mathrm{c}=0.015, \mathrm{CHCl}_{3}\right) ;{ }^{1} \mathrm{H} \mathrm{NMR}\left(600 \mathrm{MHz}, \mathrm{CDCl}_{3}\right) \delta$ $7.99-7.92(\mathrm{~m}, 2 \mathrm{H}, \mathrm{Ar}), 7.93-7.85(\mathrm{~m}, 2 \mathrm{H}, \mathrm{Ar}), 7.63-7.49(\mathrm{~m}, 4 \mathrm{H}, \mathrm{Ar}), 7.45-7.31(\mathrm{~m}, 12 \mathrm{H}$, Ar), $7.29-7.12(\mathrm{~m}, 2 \mathrm{H}, \mathrm{Ar}), 6.91(\mathrm{~d}, J=9.1 \mathrm{~Hz}, 2 \mathrm{H}, \mathrm{Ar}), 6.75(\mathrm{~d}, J=9.1 \mathrm{~Hz}, 2 \mathrm{H}, \operatorname{Ar}), 6.36(\mathrm{~d}, J$ $=8.8 \mathrm{~Hz}, 1 \mathrm{H}, \mathrm{NH}), 5.75(\mathrm{t}, J=9.2 \mathrm{~Hz}, 1 \mathrm{H}, \mathrm{H}-3 \mathrm{~b}), 5.65(\mathrm{dd}, J=9.3,6.9 \mathrm{~Hz}, 1 \mathrm{H}, \mathrm{H}-2 \mathrm{~b}), 5.50(\mathrm{~s}$, 1H, PhCH), 5.32 (d, $J=3.3 \mathrm{~Hz}, 1 \mathrm{H}, \mathrm{H}-1 \mathrm{a}), 5.24$ (d, $J=7.7 \mathrm{~Hz}, 1 \mathrm{H}, \mathrm{H}-1 \mathrm{~b}), 5.22$ (d, $J=12.1 \mathrm{~Hz}$, 1H, $\mathrm{PhCHH}), 5.17$ (d, $J=12.1 \mathrm{~Hz}, 1 \mathrm{H}, \mathrm{PhCHH}), 4.69$ (t, $J=8.8 \mathrm{~Hz}, 1 \mathrm{H}, \mathrm{H}-4 \mathrm{a}), 4.50-4.39$ (m, 1H, H-2a), 4.28 (d, $J=8.8 \mathrm{~Hz}, 1 \mathrm{H}, \mathrm{H}-5 \mathrm{~b}), 4.20$ (d, $J=10.7,1 \mathrm{H}, \mathrm{H}-6 \mathrm{a}), 4.07-4.02$ (m, 2H, H-3a, H-4b), 3.95 (d, $J=10.6,1 \mathrm{H}, \mathrm{H}-6 \mathrm{a}), 3.75$ (s, 3H, OCH $H_{3}$ ), 3.68 (s, 1H, H-5a), 0.84 (s, 9H, TBS), 0.09 (s, 3H, TBS), 0.07 (s, 3H, TBS); ${ }^{13} \mathrm{C}$ NMR (151 MHz, $\left.\mathrm{CDCl}_{3}\right) \delta 167.0\left(\mathrm{CO}_{2} \mathrm{Bn}\right), 165.9(\mathrm{COPh})$, 165.1(COPh), $161.8(\mathrm{NHCO}), 155.9,150.8,137.6,134.8,133.9,133.5,130.2,129.8,128.9,128.8$, 128.8, 128.7,128.6,128.6,128.5, 128.5, 128.2, 126.2, 118.9, 114.6, $100.9(\mathrm{PhCH}), 100.7(\mathrm{C}-1 \mathrm{a}$ GalN), 98.0 (C-1b GluA), $92.0\left(\mathrm{CCl}_{3}\right), 76.0$ (C-4b), 75.6 (C-5b), 74.6 (C-4a), 73.7 (C-3b), 71.8 (C-2b), 69.1 (C-6a), $68.0\left(\mathrm{PhCH}_{2}\right), 67.8$ (C-3a), $64.3(\mathrm{C}-5 \mathrm{a}), 55.7\left(\mathrm{OCH}_{3}\right), 51.8(\mathrm{C}-2 \mathrm{a}), 25.7$ (TBS), 18.0 (TBS), -4.1 (TBS), -4.5 (TBS); HRMS (ESI) calcd. for $\mathrm{C}_{55} \mathrm{H}_{58} \mathrm{O}_{15} \mathrm{NCl}_{3} \mathrm{SiNa}(\mathrm{M}+\mathrm{Na})^{+}$ 1128.2534 , found $1128.2566 \mathrm{~m} / \mathrm{z}$. 
phenyl (4,6-O-benzylidene-3- $O$-acetyl-2-deoxy-2-trichloroacetamido-1-thio- $\beta$-Dgalactopyranoside (19):
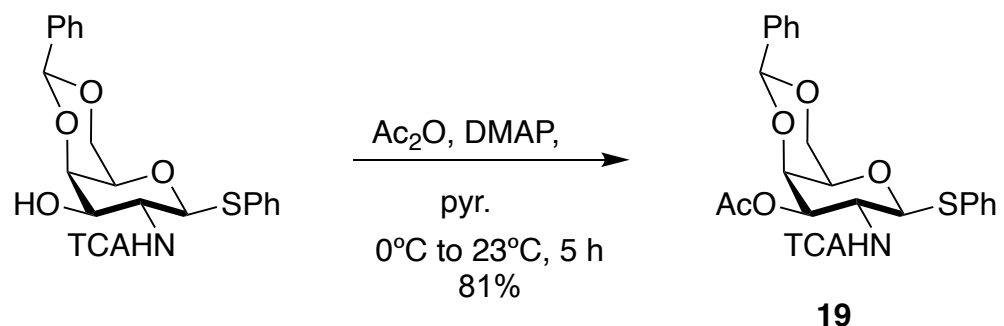

To a solution of known phenyl 4,6-O-benzylidene acetal-2-deoxy-2-trichloracetamido-1-thio- $\beta$ D-galactopyranoside ${ }^{6}(4.0 \mathrm{~g}, 7.92 \mathrm{mmol}, 1$ equiv. $)$ in pyridine $(35 \mathrm{~mL})$ was cooled to $0{ }^{\circ} \mathrm{C}$ under an argon atmosphere and added acetic anhydride $(3.7 \mathrm{~mL}, 39.6 \mathrm{mmol}, 5$ equiv.) and DMAP $(0.1$ equiv.). The reaction was stirred at room temperature. At $1 \mathrm{~h}$, TLC indicated that the starting material was completely consumed. The reaction was cooled to $0{ }^{\circ} \mathrm{C}$ and the excess acetic anhydride was quenched by the addition of $\mathrm{CH}_{3} \mathrm{OH}(5.0 \mathrm{~mL})$. After 5 min at $0{ }^{\circ} \mathrm{C}$, the crude reaction mixture was stirred at room temperature for $5 \mathrm{~min}$ and then transferred to a separatory funnel with EtOAc $(100 \mathrm{~mL})$. The reaction was washed with $1 \mathrm{~N} \mathrm{HCl}(3 \times 25 \mathrm{~mL})$, satd. aq. $\mathrm{NaHCO}_{3}(1 \times 25 \mathrm{~mL})$, brine $(1 \times 125 \mathrm{~mL})$, dried $\left(\mathrm{NaSO}_{4}\right)$ and filtered through a glass fritted vacuum filter funnel to remove drying agent. The filtrate was concentrated to a crude oil which was purified by flash column chromatography (1:3 to $1: 1$ EtOAc/hexanes) then was crystalized from hot EtOAc and hexanes to give product $(3.50 \mathrm{~g}, 6.40 \mathrm{mmol}, 81 \%)$ as white crystals. $\mathrm{R}_{\mathrm{f}}=0.55$ $\left(\right.$ EtOAc/Hexane 1:1) visualized with ceric ammonium molybdate stain; $[\alpha] \mathrm{D}^{25}=+4.2^{\circ}(\mathrm{c}=$ 0.0012, $\left.\mathrm{CHCl}_{3}\right) ;{ }^{1} \mathrm{H}$ NMR (600 MHz, $\left.\mathrm{CDCl}_{3}\right) \delta 7.69-7.62(\mathrm{~m}, 2 \mathrm{H}, \mathrm{Ar}), 7.45-7.42(\mathrm{~m}, 2 \mathrm{H}, \mathrm{Ar})$, $7.41-7.35$ (m, 3H, Ar), 7.35-7.31 (m, 1H, Ar), $7.29-7.26$ (m, 2H, Ar), 6.69 (d, $J=8.3,1 \mathrm{H}, \mathrm{NH})$, 5.53 (s, 1H, PhCH), 5.44 (dd, $J=10.9,3.3 \mathrm{~Hz}, 1 \mathrm{H}, \mathrm{H}-3), 5.18$ (d, $J=10.0 \mathrm{~Hz}, 1 \mathrm{H}, \mathrm{H}-1), 4.40$ (dd, $J=12.3,1.7 \mathrm{~Hz}, 1 \mathrm{H}, \mathrm{H}-6), 4.37$ (dd, $J=3.3,1.0 \mathrm{~Hz}, 1 \mathrm{H}, \mathrm{H}-4), 4.14$ (td, $J=10.5,8.3 \mathrm{~Hz}, 1 \mathrm{H}, \mathrm{H}-$ 2), $4.06(\mathrm{dd}, J=12.4,1.6 \mathrm{~Hz}, 1 \mathrm{H}, \mathrm{H}-6), 3.67$ (s, $1 \mathrm{H}, \mathrm{H}-5), 2.03\left(\mathrm{~s}, 3 \mathrm{H}, \mathrm{COCH}_{3}\right) ;{ }^{13} \mathrm{C} \mathrm{NMR}(151$ 
$\left.\mathrm{MHz}, \mathrm{CDCl}_{3}\right) \delta 170.7\left(\mathrm{COCH}_{3}\right), 161.4(\mathrm{NHCO}), 137.6,134.0,130.9,129.3,129.1,128.6,128.3$, 126.5, $101.0\left(\mathrm{PhCH} /\right.$ benzylidene), $92.4\left(\mathrm{CCl}_{3}\right), 84.4$ (C-1), 73.4 (C-4), 70.9 (C-3), 70.0 (C-5), 69.3 (C-6), $50.9(\mathrm{C}-2), 20.9\left(\mathrm{COCH}_{3}\right)$; HRMS (ESI) calcd. for $\mathrm{C}_{23} \mathrm{H}_{22} \mathrm{O}_{6} \mathrm{Cl}_{3} \mathrm{NSNa}(\mathrm{M}+\mathrm{Na})^{+}$568.0133, found $568.0126 \mathrm{~m} / \mathrm{z}$.

$p$-Methoxyphenyl $(4,6-O$-benzylidene-3- $O$-acetyl-2-deoxy-2-trichloroacetamido- $\beta$-D-
galactopyranosyl) $-(1 \rightarrow 4)$-(methyl 2,3 -di- $O$-benzoyl- $\beta$-D-glucopyranosid)uronate $(20)$ :

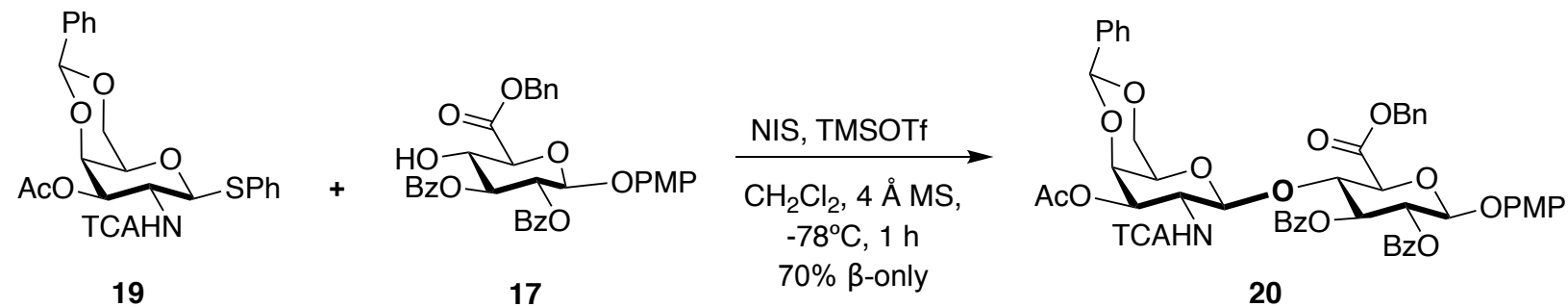

A solution of acceptor $\mathbf{1 7 ^ { 5 }}(0.90 \mathrm{~g}, 1.50 \mathrm{mmol}, 1.0$ equiv. $)$ and donor $\mathbf{1 9}^{5}(0.95 \mathrm{~g}, 1.73 \mathrm{mmol}, 1.1$ equiv.) in anhydrous $\mathrm{CH}_{2} \mathrm{Cl}_{2}(20 \mathrm{~mL})$ under an argon atmosphere was added $4 \AA \mathrm{MS}(0.50 \mathrm{~g})$ at room temperature and allowed to stir for $1 \mathrm{~h}$. The reaction mixture was cooled to $-78^{\circ} \mathrm{C}$ and added NIS (0.52 g, 3.0 mmol, 2.0 equiv.). After 1 min, a solution of TMSOTf ( 0.15 equiv., $2.0 \mathrm{~mL}, 5 \%$ in anhydrous $\mathrm{CH}_{2} \mathrm{Cl}_{2}$ ) was added dropwise. The reaction was stirred at $-78^{\circ} \mathrm{C}$ for $2 \mathrm{~h}$ when TLC indicated complete consumption of the alcohol $\mathbf{3 1}$ starting material. The reaction was quench by the addition of dilute $\mathrm{Et}_{3} \mathrm{~N}$ in $\mathrm{CH}_{2} \mathrm{Cl}_{2}$ while warming to room temperature. The reaction was filtered through a glass fritted filter funnel equipped with a pad of Celite to remove the $4 \AA$ MS and then concentrated in vacuo. The crude oil was then diluted with EtOAc and transferred to a separatory funnel and washed with satd. aq. $\mathrm{Na}_{2} \mathrm{~S}_{2} \mathrm{O}_{3}(20 \mathrm{~mL} \times 1)$, brine $(20 \mathrm{~mL} \times 1)$, and dried over anhydrous $\mathrm{Na}_{2} \mathrm{SO}_{4}$. The mixture was filtered and concentrated to a crude oil. Flash chromatography of the crude material eluting with EtOAc/Hexane (2:3) gave separable $\beta$ product:(1.10 g, $1.06 \mathrm{mmol}, 70 \%)$ as white foam. The $\beta$-anomer had $\mathrm{R}_{\mathrm{f}}=0.45$ (EtOAc/Hexane 
2:3) visualized with ceric ammonium molybdate stain; $[\alpha] \mathrm{D}^{25}=+17.3^{\circ}\left(\mathrm{c}=0.012, \mathrm{CHCl}_{3}\right) ;{ }^{1} \mathrm{H}$ NMR $\left(600 \mathrm{MHz}, \mathrm{CDCl}_{3}\right) \delta$ 7.97-7.92 (m, 4H, Ar), $7.48-7.24(\mathrm{~m}, 14 \mathrm{H}, \mathrm{Ar}), 7.20$ (t, J= 7.8 Hz, 2H, Ar), 6.92 (d, $J=9.1 \mathrm{~Hz}, 2 \mathrm{H}, \mathrm{Ar}), 6.71$ (d, $J=9.2 \mathrm{~Hz}, 2 \mathrm{H}, \mathrm{Ar}), 5.77$ (t, $J=9.1 \mathrm{~Hz}, 1 \mathrm{H}, \mathrm{H}-3 \mathrm{~b})$, $5.61(\mathrm{dd}, J=9.2,7.0 \mathrm{~Hz}, 1 \mathrm{H}, \mathrm{H}-2 \mathrm{~b}), 5.30$ (d, $J=12.4 \mathrm{~Hz}, 1 \mathrm{H}, \mathrm{PhCH} H), 5.28$ (s, 1H, PhCH), 5.21 (d, $J=7.0 \mathrm{~Hz}, 1 \mathrm{H}, \mathrm{H}-1 \mathrm{a}), 5.14(\mathrm{~d}, J=12.2 \mathrm{~Hz}, 1 \mathrm{H}, \mathrm{PhCHH}), 5.03$ (dd, $J=11.2,3.4 \mathrm{~Hz}, 1 \mathrm{H}, \mathrm{H}-$ 3a), 4.85 (d, $J=8.3 \mathrm{~Hz}, 1 \mathrm{H}, \mathrm{H}-1 \mathrm{~b}), 4.50$ (t, $J=9.1 \mathrm{~Hz}, 1 \mathrm{H}, \mathrm{H}-4 \mathrm{~b}), 4.28$ (d, $J=9.2 \mathrm{~Hz}, 1 \mathrm{H}, \mathrm{H}-5 \mathrm{~b})$, $4.18-4.11(\mathrm{~m}, 1 \mathrm{H}, \mathrm{H}-2 \mathrm{a}), 4.10(\mathrm{~d}, J=3.4 \mathrm{~Hz}, 1 \mathrm{H}, \mathrm{H}-4 \mathrm{a}), 3.73\left(\mathrm{~s}, 1 \mathrm{H}, \mathrm{OCH}_{3}\right), 3.65$ (d, $J=12.5$ Hz, 1H, H-6a), 3.60 (d, $J=12.4$ Hz, 1H, H-6a), 3.09 (s, 1H, H-5a), 2.02 (s, 3H, COCH $) ;{ }^{13} \mathrm{C} \mathrm{NMR}$ $\left(151 \mathrm{MHz}, \mathrm{CDCl}_{3}\right) \delta 170.9\left(\mathrm{COCH}_{3}\right), 168.1\left(\mathrm{CO}_{2} \mathrm{Bn}\right), 165.5(\mathrm{COPh}), 165.3(\mathrm{COPh}), 161.6$ (NHCO), 155.9, 150.8, 137.6, 134.9, 133.4, 132.9, 130.0, 129.9, 129.0, 128.8, 128.8, 128.7, 128.5, 128.3, 128.2 128.0, 126.6, 119.0, 114.6, 100.9 (PhCH), 100.8 (C-1a GalN), 100.1 (C-1b GlcA), $92.6\left(\mathrm{CCl}_{3}\right), 76.1$ (C-4b), 74.4 (C-5b), 72.8 (C-4a), 72.5(C-3b), 72.0 (C-2b), 70.7 (C-3a), 68.1 (C6a), $67.9\left(\mathrm{PhCH}_{2}\right), 66.5(\mathrm{C}-5 \mathrm{a}), 55.7\left(\mathrm{OCH}_{3}\right), 52.6(\mathrm{C}-2 \mathrm{a}), 20.8\left(\mathrm{COCH}_{3}\right)$; HRMS (ESI) calcd. for $\mathrm{C}_{51} \mathrm{H}_{46} \mathrm{O}_{16} \mathrm{Cl}_{3} \mathrm{NNa}(\mathrm{M}+\mathrm{Na})^{+} 1056.1774$, found $1056.1789 \mathrm{~m} / \mathrm{z}$. 
$p$-Methoxyphenyl methyl 2,3-di- $O$-benzyl- $\beta$-D-glucopyranosidurinate (31):
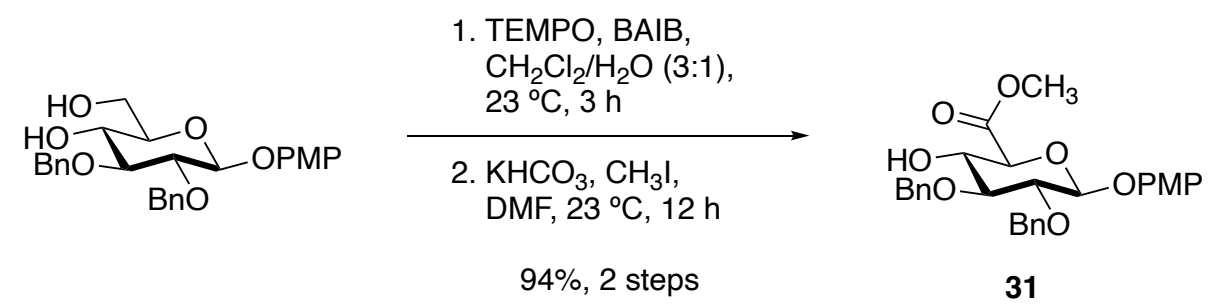

To a solution of known $\operatorname{diol}^{4 \mathrm{~b}}\left(3.75 \mathrm{~g}, 8.04 \mathrm{mmol}, 1.0\right.$ equiv.) in $\mathrm{CH}_{2} \mathrm{Cl}_{2} / \mathrm{H}_{2} \mathrm{O}(3 / 1 \mathrm{v} / \mathrm{v}, 100 \mathrm{~mL})$ under an argon atmosphere was added 2,2,6,6-tetramethyl-1-piperidinyloxy (TEMPO) (377 mg, $2.41 \mathrm{mmol}, 0.3$ equiv.) and (Diacetoxyiodo) benzene (BAIB) (6.45g, $20.1 \mathrm{mmol}, 2.5$ equiv.). The biphasic mixture was stirred vigorously for $3 \mathrm{~h}$ and quenched by the addition of satd. aq. $\mathrm{Na}_{2} \mathrm{~S}_{2} \mathrm{O}_{3}$ $(20 \mathrm{~mL})$. The layers were separated, and the aqueous layer was acidified with $1 \mathrm{M}$ aqueous $\mathrm{HCl}$ to pH 2 and extracted with $\mathrm{CH}_{2} \mathrm{Cl}_{2}(50 \mathrm{~mL} \times 3$. The combined organic layers were dried over anhydrous $\mathrm{Na}_{2} \mathrm{SO}_{4}$, filtered, and concentrated in vacuo. The residue was dissolved in $65.0 \mathrm{~mL}$ of anhydrous DMF. To this solution was added sequentially $\mathrm{KHCO}_{3}(3.23 \mathrm{~g}, 32.3 \mathrm{mmol}, 5.0$ equiv.) and $\mathrm{CH}_{3} \mathrm{I}(0.44 \mathrm{~mL}, 7.10 \mathrm{mmol}, 1.1$ equiv. $)$ at $0{ }^{\circ} \mathrm{C}$ and the solution was stirred at $25^{\circ} \mathrm{C}$ for about $12 \mathrm{~h}$. The solvents were concentrated in vacuo, and the residue was purified by flash silica gel chromatography (50:50 EtOAc/Hexanes) to provide the glucuronic acid methyl ester 31 (3.00 g, $6.45 \mathrm{mmol}, 94 \%$ yield) as a white amorphous solid. Glucuronic acid methyl ester had $\mathrm{R}_{\mathrm{f}} 0.40$ $\left(\right.$ EtOAc/Hexane 1:1) visualized with ceric ammonium molybdate stain; $[\alpha] \mathrm{D}^{25}=+9.2^{\circ}(\mathrm{c}=$ $\left.0.0024, \mathrm{CHCl}_{3}\right) ;{ }^{1} \mathrm{H} \mathrm{NMR}\left(600 \mathrm{MHz}, \mathrm{CDCl}_{3}\right) \delta 7.38-7.28(\mathrm{~m}, 10 \mathrm{H}, \mathrm{Ar}), 7.02(\mathrm{~d}, J=9.0 \mathrm{~Hz}, 2 \mathrm{H}$, Ar), 6.84 (d, $J=9.0 \mathrm{~Hz}, 2 \mathrm{H}, \mathrm{Ar}), 5.02$ (d, $J=10.9 \mathrm{~Hz}, 1 \mathrm{H}, \mathrm{PhCHH}), 4.93$ (d, $J=7.7 \mathrm{~Hz}, 1 \mathrm{H}, \mathrm{H}-$ 1), 4.92 (d, $J=11.4 \mathrm{~Hz}, 1 \mathrm{H}, \mathrm{PhCH} H), 4.85$ (d, $J=11.4 \mathrm{~Hz}, 1 \mathrm{H}, \mathrm{PhCHH}), 4.82$ (d, $J=10.9 \mathrm{~Hz}$, 1H, $\mathrm{PhCH} H$ ), $3.99-3.94$ (m, 1H, H-4), 3.91 (m, 1H, H-5), 3.81 (s, 3H, $\left.\mathrm{CO}_{2} \mathrm{CH}_{3}\right), 3.79$ (s, 3H, $\left.\mathrm{OCH}_{3}\right), 3.74-3.68(\mathrm{~m}, 1 \mathrm{H}, \mathrm{H}-2), 3.59$ (t, $\left.J=8.9 \mathrm{~Hz}, 1 \mathrm{H}, \mathrm{H}-3\right), 2.88(\mathrm{~s}, 1 \mathrm{H}, \mathrm{OH}) ;{ }^{13} \mathrm{C}$ NMR $(151$ $\left.\mathrm{MHz}, \mathrm{CDCl}_{3}\right) \delta 169.6\left(\mathrm{CO}_{2} \mathrm{CH}_{3}\right), 155.7,151.3,138.5,138.1,128.6,128.5,128.3,128.1,128.0$ 
118.7, 114.7, 103.2 (C-1), 83.1 (C-3), $81.0(\mathrm{C}-2), 75.6\left(\mathrm{PhCH}_{2}\right), 75.26\left(\mathrm{PhCH}_{2}\right), 74.2(\mathrm{C}-5), 71.7$

(C-4), $55.8\left(\mathrm{OCH}_{3}\right), 52.9\left(\mathrm{CO}_{2} \mathrm{CH}_{3}\right)$; HRMS (ESI) calcd. for $\mathrm{C}_{28} \mathrm{H}_{30} \mathrm{O}_{8} \mathrm{Na}(\mathrm{M}+\mathrm{Na})^{+}$517.1833, found $517.1836 \mathrm{~m} / \mathrm{z}$.

$p$-Methoxyphenyl (4,6- $O$-benzylidene-3- $O$-acetyl-2-deoxy-2-trichloroacetamido- $\beta$-Dgalactopyranosyl)-(1 $\rightarrow 4)$-(methyl 2,3-di- $O$-benzyl- $\beta$-D-glucopyranosid)uronate (32):

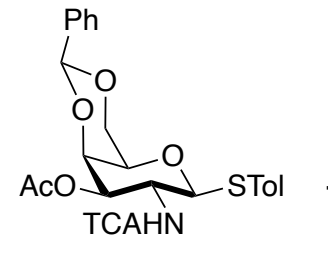

30

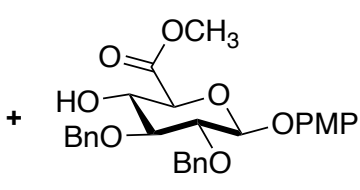

31

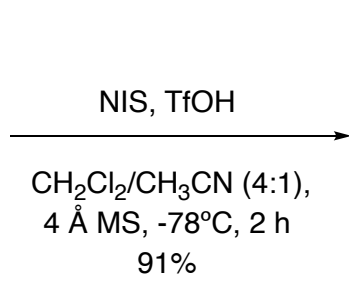

$4: 1(\beta: \alpha)$

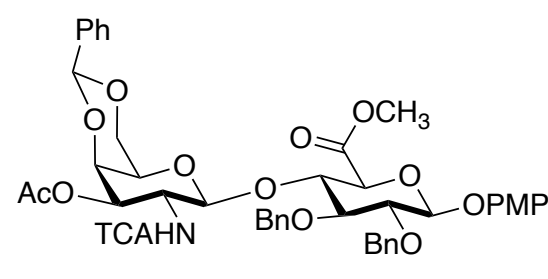

32

A solution of acceptor 31 (1.75 g, $3.50 \mathrm{mmol}, 1.0$ equiv.) and donor $\mathbf{3 0}^{7}$ (2.46 g, $4.36 \mathrm{mmol}, 1.5$ equiv.) in anhydrous $\mathrm{CH}_{2} \mathrm{Cl}_{2}(30 \mathrm{~mL})$ under an argon atmosphere was added $4 \AA \mathrm{MS}(5.0 \mathrm{~g})$ at room temperature and allowed to stir for $1 \mathrm{~h}$. The reaction mixture was cooled to $-78^{\circ} \mathrm{C}$ and added NIS (1.59 g, $7.08 \mathrm{mmol}, 2.0$ equiv.). After $1 \mathrm{~min}$, a solution of TMSOTf (0.15 equiv., $2.0 \mathrm{~mL}, 5 \%$ in anhydrous $\mathrm{CH}_{2} \mathrm{Cl}_{2}$ ) was added dropwise. The reaction was stirred at $-78^{\circ} \mathrm{C}$ for $2 \mathrm{~h}$ when TLC indicated complete consumption of the alcohol $\mathbf{3 1}$ starting material. The reaction was quench by the addition of dilute $\mathrm{Et}_{3} \mathrm{~N}$ in $\mathrm{CH}_{2} \mathrm{Cl}_{2}$ while warming to room temperature. The reaction was filtered through a glass fritted filter funnel equipped with a pad of Celite to remove the $4 \AA$ MS and then concentrated in vacuo. The crude oil was then diluted with EtOAc and transferred to a separatory funnel and washed with satd. aq. $\mathrm{Na}_{2} \mathrm{~S}_{2} \mathrm{O}_{3}(20 \mathrm{~mL} \times 1)$, brine $(20 \mathrm{~mL} \times 1)$, and dried over anhydrous $\mathrm{Na}_{2} \mathrm{SO}_{4}$. The mixture was filtered and concentrated to a crude oil. Flash chromatography of the crude material eluting with EtOAc/Hexane (1:4) gave separable $\beta / \alpha$ (4:1) products: $\beta 32(2.40 \mathrm{~g}, 2.53 \mathrm{mmol}, 74 \%)$ and $\alpha(0.60 \mathrm{~g}, 0.64 \mathrm{mmol}, 18 \%)$ as both yellow foams. The combine yield is $91 \%$. The $\beta$-anomer had $\mathrm{R}_{\mathrm{f}}=0.45$ (EtOAc/Hexane 1:1) and $\alpha$-anomer had 
$\mathrm{R}_{\mathrm{f}}=0.55$ (EtOAc/Hexane 1:1), both visualized with ceric ammonium molybdate stain; $\beta$-anomer: $[\alpha] \mathrm{D}^{25}=+46.7^{\circ}\left(\mathrm{c}=0.0018, \mathrm{CHCl}_{3}\right) ;{ }^{1} \mathrm{H} \mathrm{NMR}\left(600 \mathrm{MHz}, \mathrm{CDCl}_{3}\right) \delta 7.55-7.47(\mathrm{~m}, 2 \mathrm{H}, \mathrm{Ar})$, $7.44-7.38$ (m, 2H, Ar), $7.37-7.26$ (m, 9H, Ar), 7.17 (m, 2H,Ar), 6.97 (d, J=9.1 Hz, 2H, Ar), $6.85(\mathrm{~d}, J=9.0 \mathrm{~Hz}, 1 \mathrm{H}, \mathrm{NH}), 6.82(\mathrm{~d}, J=9.1 \mathrm{~Hz}, 2 \mathrm{H}, \mathrm{Ar}), 5.55(\mathrm{~s}, 1 \mathrm{H}, \mathrm{PhCH}), 5.20$ (d, $J=10.7$ $\mathrm{Hz}, 1 \mathrm{H}, \mathrm{PhCHH}), 5.13$ (dd, $J=11.2,3.4 \mathrm{~Hz}, 1 \mathrm{H}, \mathrm{H}-3 \mathrm{a}), 5.02$ (d, $J=8.5 \mathrm{~Hz}, 1 \mathrm{H}, \mathrm{H}-1 \mathrm{a}), 4.95$ (d, $J$ $=6.8 \mathrm{~Hz}, 1 \mathrm{H}, \mathrm{H}-1 \mathrm{~b}), 4.94(\mathrm{~d}, J=11.0 \mathrm{~Hz}, 1 \mathrm{H}, \mathrm{PhCHH}), 4.79(\mathrm{~d}, J=11.1 \mathrm{~Hz}, 1 \mathrm{H}, \mathrm{PhCH} H), 4.76$ $(\mathrm{d}, J=10.7 \mathrm{~Hz}, 1 \mathrm{H}, \mathrm{PhCH} H), 4.42(\mathrm{dt}, J=11.2,8.6 \mathrm{~Hz}, 1 \mathrm{H}, \mathrm{H}-2 \mathrm{a}), 4.31$ (d, $J=2.4 \mathrm{~Hz}, 1 \mathrm{H}, \mathrm{H}-$ 4a), 4.26 (d, $J=10.9 \mathrm{~Hz}, 1 \mathrm{H}, \mathrm{H}-6 \mathrm{a}), 4.21$ (t, $J=9.0 \mathrm{~Hz}, 1 \mathrm{H}, \mathrm{H}-4 \mathrm{~b}), 3.99$ (d, $J=10.8 \mathrm{~Hz}, 1 \mathrm{H}, \mathrm{H}-$ 6a), 3.97 (d, $J=9.4 \mathrm{~Hz}, 1 \mathrm{H}, \mathrm{H}-5 \mathrm{~b}), 3.78\left(\mathrm{~s}, 3 \mathrm{H}, \mathrm{OCH}_{3}\right), 3.75\left(\mathrm{~s}, 3 \mathrm{H}, \mathrm{CO}_{2} \mathrm{CH}_{3}\right), 3.73$ (d, $J=8.7$ $\mathrm{Hz}, 1 \mathrm{H}, \mathrm{H}-3 \mathrm{~b}), 3.69$ (dd, $J=8.9,6.8 \mathrm{~Hz}, 1 \mathrm{H}, \mathrm{H}-2 \mathrm{~b}), 3.50$ (s, $1 \mathrm{H}, \mathrm{H}-5 \mathrm{a}), 2.08\left(\mathrm{~s}, 3 \mathrm{H}, \mathrm{COCH}_{3}\right) ;{ }^{13} \mathrm{C}$ NMR (151 MHz, $\left.\mathrm{CDCl}_{3}\right) \delta 171.2\left(\mathrm{COCH}_{3}\right), 170.1\left(\mathrm{CO}_{2} \mathrm{CH}_{3}\right), 161.9(\mathrm{NHCO}), 155.6,151.2,138.6$, $138.2,137.7,129.1,128.6,128.5,128.3,128.2,128.1,127.8,127.5,126.5,118.5,118.4,114.7$, 102.7 (C-1a GalN), $101.0(\mathrm{PhCH}), 100.6$ (C-1b GlcA), $92.7\left(\mathrm{CCl}_{3}\right), 82.2$ (C-3b), 81.3 (C-2b), 78.5 (C-4b), $76.39\left(\mathrm{PhCH}_{2}\right), 75.0\left(\mathrm{PhCH}_{2}\right), 74.1$ (C-5b), 73.2 (C-4a), 71.4 (C-3a), 68.8 (C-6a), 66.9 (C5a), $55.8\left(\mathrm{OCH}_{3}\right), 53.1\left(\mathrm{CO}_{2} \mathrm{CH}_{3}\right), 52.7(\mathrm{C}-2 \mathrm{a}), 20.9\left(\mathrm{COCH}_{3}\right)$; HRMS (ESI) calcd. for $\mathrm{C}_{45} \mathrm{H}_{46} \mathrm{O}_{14} \mathrm{NCl}_{3} \mathrm{Na}(\mathrm{M}+\mathrm{Na})^{+} 952.1876$, found $952.1877 \mathrm{~m} / z$. 


\section{p-Methoxyphenyl (4,6-di-O-benzylidene-2-deoxy-2-trichloroacetamido- $\beta$-D-}

galactopyranosyl) -(1 $\rightarrow 4)$-(methyl $2,3-$ di- $O$-benzyl- $\beta$-D-glucopyranosid)uronate $(33)$ :

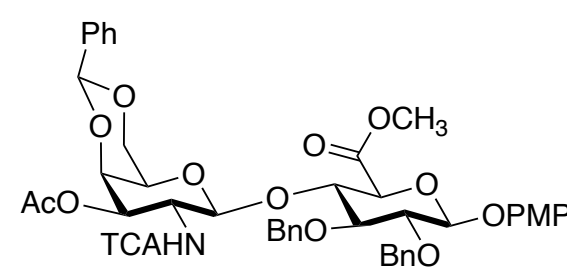

32

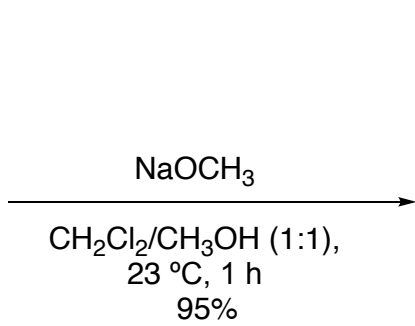

$95 \%$

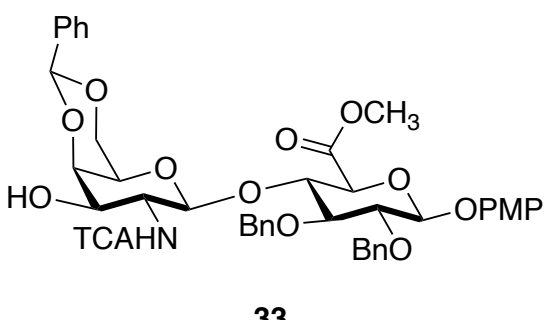

33

A solution of 32 (1.40 g, $1.54 \mathrm{mmol}, 1.0$ equiv.) in anhydrous $\mathrm{CH}_{3} \mathrm{OH} / \mathrm{CH}_{2} \mathrm{Cl}_{2}(20 \mathrm{~mL})$ under an argon atmosphere was added $\mathrm{NaOCH}_{3}$ solution $\left(1.00 \mathrm{~mL}, 1.5 \mathrm{mmol},[1.5 \mathrm{M}]\right.$ in $\left.\mathrm{CH}_{3} \mathrm{OH}\right)$ dropwise at room temperature. At $1 \mathrm{~h}$, the $\mathrm{pH}$ of the reaction mixture was still $>12$ and TLC indicated that the starting material had been completely consumed for a more polar spot. The reaction was neutralized by the addition of acidic form Amberlite IR $120(\mathrm{H}+)$ ion exchange resin. The solution was filtered through a glass fritted funnel with a pad of Celite to remove the resin. The filtrate was concentrated in vacuo to give 33 (1.28 g, $1.44 \mathrm{mmol},>95 \%)$ as a yellow foam in quantitative yield. $\mathrm{Rf}=0.25($ EtOAc/Hexanes $1: 1)$ visualized with ceric ammonium molybdate stain; $[\alpha] \mathrm{D}^{25}=+12.8^{\circ}$ $\left(\mathrm{c}=0.0015, \mathrm{CHCl}_{3}\right) ;{ }^{1} \mathrm{H} \mathrm{NMR}\left(600 \mathrm{MHz}, \mathrm{CDCl}_{3}\right) \delta 7.54-7.43(\mathrm{~m}, 4 \mathrm{H}, \mathrm{Ar}), 7.40-7.26(\mathrm{~m}, 9 \mathrm{H}$, Ar), $7.26-7.14$ (m, 2H, Ar), 6.99 (d, $J=9.1 \mathrm{~Hz}, 2 \mathrm{H}, \mathrm{Ar}), 6.84$ (d, J=9.1 Hz, 2H, Ar), 5.59 (s, 1H, PhCH), $5.21(\mathrm{~d}, J=10.4 \mathrm{~Hz}, 1 \mathrm{H}, \mathrm{PhCHH}), 4.96(\mathrm{~d}, J=6.74,1 \mathrm{H}, \mathrm{H}-1 \mathrm{~b}), 4.95$ (d, $J=11.0 \mathrm{~Hz}$, 1H, PhCHH), 4.86 (d, $J=8.4$ Hz, 1H, H-1a), 4.81 (d, $J=11.0 \mathrm{~Hz}, 1 \mathrm{H}, \mathrm{PhCH} H), 4.75$ (d, $J=10.4$ $\mathrm{Hz}, 1 \mathrm{H}, \mathrm{PhCH} H), 4.26$ (d, $J=12.7 \mathrm{~Hz}, 1 \mathrm{H}, \mathrm{H}-6 \mathrm{a}), 4.24-4.16$ (m, 2H, H-2a, H-4a), 4.14 (t, $J=$ $9.0 \mathrm{~Hz}, 1 \mathrm{H}, \mathrm{H}-4 \mathrm{~b}), 4.03-4.00$ (m, 2H, H-6a, H-5b), 3.83-3.79 (m, 1H, H-3a), 3.78 (s, 3H, OCH $)$, 3.77 (s, 3H, $\left.\mathrm{CO}_{2} \mathrm{CH}_{3}\right), 3.74-3.69$ (m, 2H, H-2b, H-3b), 3.48 (s, 1H, H-5a), $2.78(\mathrm{~s}, 1 \mathrm{H}, \mathrm{OH}) ;{ }^{13} \mathrm{C}$ NMR (151 MHz, $\left.\mathrm{CDCl}_{3}\right) \delta 170.7\left(\mathrm{CO}_{2} \mathrm{CH}_{3}\right), 163.3(\mathrm{NHCO}), 155.7,151.2,138.5,138.1,137.6$, $129.3,128.7,128.5,128.3,128.1,127.9,127.7,126.5,126.2,118.5,114.7,102.73(\mathrm{PhCH}), 101.44$ (C-1b GlcA), 100.7 (C-1a GalN), $92.8\left(\mathrm{CCl}_{3}\right), 82.3$ (C-3b), 81.3 (C-2b), 78.9 (C-4b), 76.6 
( $\left.\mathrm{PhCH}_{2}\right), 75.1\left(\mathrm{PhCH}_{2}\right), 75.0(\mathrm{C}-4 \mathrm{a}), 74.0(\mathrm{H}-5 \mathrm{~b}), 72.6$ (H-3a), 68.7 (C-6a), 67.2 (C-5a), 55.8

$\left(\mathrm{OCH}_{3}\right), 55.71\left(\mathrm{CO}_{2} \mathrm{CH}_{3}\right), 53.2(\mathrm{C}-2 \mathrm{a})$; HRMS (ESI) calcd. for $\mathrm{C}_{43} \mathrm{H}_{44} \mathrm{O}_{13} \mathrm{NCl}_{3} \mathrm{Na}(\mathrm{M}+\mathrm{Na})^{+}$ 910.1770, found $910.1769 \mathrm{~m} / \mathrm{z}$.

$p$-Methoxyphenyl (3-O-benzoyl-4-azido-2,4,6-trideoxy-2-trichloroacetamido- $\beta$-Dgalactopyranosyl)-( $1 \rightarrow 3)-(4,6$-di- $O$-benzylidene-2-deoxy-2-trichloroacetamido- $\beta$-Dgalactopyranosyl)-(1 $\rightarrow 4)$-(methyl 2,3-di- $O$-benzyl- $\beta$-D-glucopyranosid)uronate (34):

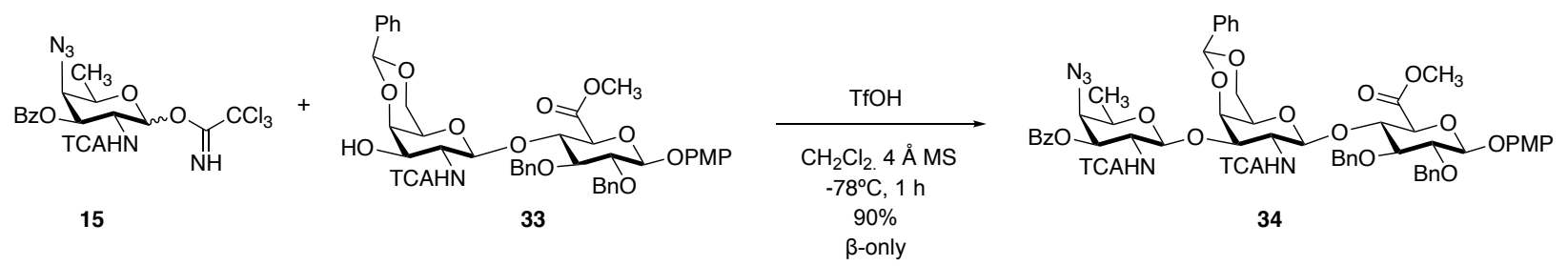

A solution of acceptor 33 (1.10 g, $1.24 \mathrm{mmol}, 1.0$ equiv.) and donor 15 (0.864 g, $1.49 \mathrm{mmol}, 1.2$ equiv.) in anhydrous $\mathrm{CH}_{2} \mathrm{Cl}_{2}(12 \mathrm{~mL})$ under an argon atmosphere was added $4 \AA \mathrm{MS}(3.0 \mathrm{~g})$ at room temperature and allowed to stir for $1 \mathrm{~h}$. The reaction mixture was cooled to $-78{ }^{\circ} \mathrm{C}$ and a solution of $\mathrm{TfOH}\left(0.15\right.$ equiv., $2.0 \mathrm{~mL}, 5 \%$ in anhydrous $\left.\mathrm{CH}_{2} \mathrm{Cl}_{2}\right)$ was added dropwise. The reaction was stirred at $-78^{\circ} \mathrm{C}$ for $1 \mathrm{~h}$ when TLC indicated complete consumption of the alcohol 33 starting material. The reaction was quench by the addition of dilute $\mathrm{Et}_{3} \mathrm{~N}$ in $\mathrm{CH}_{2} \mathrm{Cl}_{2}$ while warming to room temperature. The reaction was filtered through a glass fritted filter funnel equipped with a pad of Celite to remove the $4 \AA$ MS and then concentrated in vacuo. The crude oil was then diluted with EtOAc and transferred to a separatory funnel and washed with satd. aq. $\mathrm{Na}_{2} \mathrm{~S}_{2} \mathrm{O}_{3}(20$ $\mathrm{mL} \times 1)$, brine $(20 \mathrm{~mL} \times 1)$, and dried over anhydrous $\mathrm{Na}_{2} \mathrm{SO}_{4}$. The mixture was filtered and concentrated to a crude oil. Flash chromatography of the crude material eluting with EtOAc/Hexane (1:4) gave the $\mathbf{3 4}(1.45 \mathrm{~g}, 1.11 \mathrm{mmol}, 90 \%)$ as a white foam. The trisaccharide $\mathbf{3 5}$ had $R_{f}=0.40(E t O A c / H e x a n e ~ 1: 1)$ visualized with ceric ammonium molybdate stain; $[\alpha] D^{25}=$ $27.7^{\circ}\left(\mathrm{c}=0.0017, \mathrm{CHCl}_{3}\right) ;{ }^{1} \mathrm{H} \mathrm{NMR}\left(600 \mathrm{MHz}, \mathrm{CDCl}_{3}\right) \delta 8.07-7.98(\mathrm{~m}, 2 \mathrm{H}, \mathrm{Ar}), 7.64-7.55(\mathrm{~m}$, 1H, Ar), $7.52-7.42(\mathrm{~m}, 4 \mathrm{H}, \mathrm{Ar}), 7.40-7.34(\mathrm{~m}, 2 \mathrm{H}, \mathrm{Ar}), 7.28(\mathrm{~m}, 8 \mathrm{H}, \mathrm{Ar}), 7.20-7.11(\mathrm{~m}, 3 \mathrm{H}$, 
Ar), $7.13(\mathrm{~d}, J=7.7 \mathrm{~Hz}, 1 \mathrm{H}, \mathrm{NH}), 6.97(\mathrm{~d}, J=9.1 \mathrm{~Hz}, 2 \mathrm{H}, \mathrm{Ar}), 6.85(\mathrm{~d}, J=9.0 \mathrm{~Hz}, 1 \mathrm{H}, \mathrm{NH}), 6.82$ (d, $J=9.1 \mathrm{~Hz}, 2 \mathrm{H}, \mathrm{Ar}), 5.57$ (s, 1H, PhCH), 5.32 (dd, $J=11.0,3.5 \mathrm{~Hz}, 1 \mathrm{H}, \mathrm{H}-3 \mathrm{c}), 5.14$ (d, $J=$ $10.9 \mathrm{~Hz}, 1 \mathrm{H}, \mathrm{PhCHH}), 5.05$ (d, $J=8.3 \mathrm{~Hz}, 1 \mathrm{H}, \mathrm{H}-1 \mathrm{a}), 4.97$ (d, $J=6.6 \mathrm{~Hz}, 1 \mathrm{H}, \mathrm{H}-1 \mathrm{~b}), 4.91$ (d, $J=$ $11.0 \mathrm{~Hz}, 1 \mathrm{H}, \mathrm{PhCHH}), 4.88$ (d, $J=8.5 \mathrm{~Hz}, 1 \mathrm{H}, \mathrm{H}-1 \mathrm{c}), 4.77$ (d, $J=11.0 \mathrm{~Hz}, 1 \mathrm{H}, \mathrm{PhCH} H), 4.73$ (d, $J=11.0 \mathrm{~Hz}, 1 \mathrm{H}, \mathrm{PhCH} H), 4.46(\mathrm{dt}, J=11.0,8.7 \mathrm{~Hz}, 1 \mathrm{H}, \mathrm{H}-2 \mathrm{c}), 4.36(\mathrm{dd}, J=10.8,3.4 \mathrm{~Hz}, 1 \mathrm{H}$, H-3a), 4.32 (dd, $J=3.5,1.0 \mathrm{~Hz}, 1 \mathrm{H}, \mathrm{H}-4 \mathrm{a}), 4.28$ (m, 1H, H-4b), 4.27 (d, $J=10.7 \mathrm{~Hz}, 1 \mathrm{H}, \mathrm{H}-6 \mathrm{a})$, $4.18-4.11(\mathrm{~m}, 1 \mathrm{H}, \mathrm{H}-2 \mathrm{a}), 4.01$ (d, $J=10.7 \mathrm{~Hz}, 1 \mathrm{H}, \mathrm{H}-6 \mathrm{a}), 3.98$ (d, $J=9.1 \mathrm{~Hz}, 1 \mathrm{H}, \mathrm{H}-5 \mathrm{~b}), 3.95$ (dd, $J=3.5,1.3 \mathrm{~Hz}, 1 \mathrm{H}, \mathrm{H}-4 \mathrm{c}), 3.84$ (qd, $J=7.3,1.8 \mathrm{~Hz}, 1 \mathrm{H}, \mathrm{H}-5 \mathrm{c}), 3.78\left(\mathrm{~s}, 3 \mathrm{H}, \mathrm{OCH}_{3}\right), 3.75$ (s, $3 \mathrm{H}, \mathrm{CO}_{2} \mathrm{CH}_{3}$ ), $3.75-3.67$ (m, 2H, H-2b, H-3b), 3.46 (s, 1H, H-5a), 1.43 (d, J = 6.3 Hz, 3H, H6c); ${ }^{13} \mathrm{C}$ NMR (151 MHz, $\left.\mathrm{CDCl}_{3}\right) \delta 170.0\left(\mathrm{CO}_{2} \mathrm{CH}_{3}\right), 166.2(\mathrm{COPh}), 162.5(\mathrm{NHCO}), 162.2$ (NHCO), 155.6, 151.2, 138.6, 138.1, 137.9, 134.0, 130.3, 128.8, 128.7, 128.52, 128.49, 128.3, 128.2, 128.2, 128.1, 127.8, 127.5, 126.4, 118.4, 114.7, 102.7 (C-1b GlcA), 100.6 (PhCH), $99.6(\mathrm{C}-$ 1a GalN), 99.0 (C-1c AAT), $92.9\left(\mathrm{CCl}_{3}\right), 92.2\left(\mathrm{CCl}_{3}\right), 82.2$ (C-3b), 81.3 (C-2b), 77.6 (C-4b), 76.0 ( $\left.\mathrm{PhCH}_{2}\right), 75.2$ (C-4a), $75.0\left(\mathrm{PhCH}_{2}\right), 74.3$ (C-5b), 73.4 (C-3c), 72.4 (C-3a), 70.3 (C-5c), 68.7 (C6a), $67.1(\mathrm{C}-5 \mathrm{a}), 63.4(\mathrm{C}-4 \mathrm{c}), 55.8\left(\mathrm{OCH}_{3}\right), 54.8(\mathrm{C}-2 \mathrm{a}), 53.0\left(\mathrm{CO}_{2} \mathrm{CH}_{3}\right), 52.5(\mathrm{C}-2 \mathrm{c}), 17.6(\mathrm{C}-6 \mathrm{c})$; HRMS (ESI) calcd. for $\mathrm{C}_{58} \mathrm{H}_{56} \mathrm{O}_{17} \mathrm{~N}_{5} \mathrm{Cl}_{6}(\mathrm{M}-\mathrm{H})^{-} 1304.1808$, found $1304.1770 \mathrm{~m} / \mathrm{z}$. 
p-Methoxyphenyl (3-O-benzoyl-4-azido-2,4,6-trideoxy-2-trichloroacetamido- $\beta$-Dgalactopyranosyl)-( $\rightarrow 3)$-(6-O-benzyl-2-deoxy-2-trichloroacetamido- $\beta$-Dgalactopyranosyl)-(1 $\rightarrow 4)$-(methyl $2,3-$ di- $O$-benzyl- $\beta$-D-glucopyranosid)uronate $(35)$ :

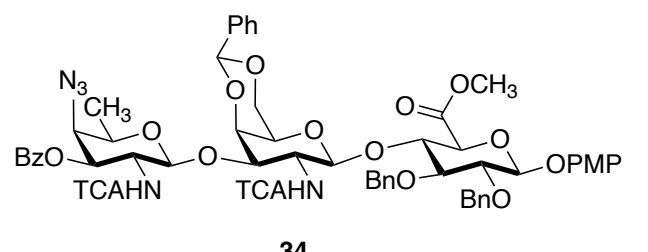

34

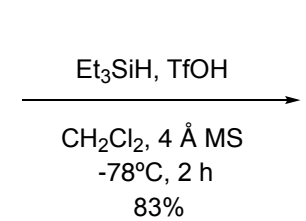

$83 \%$

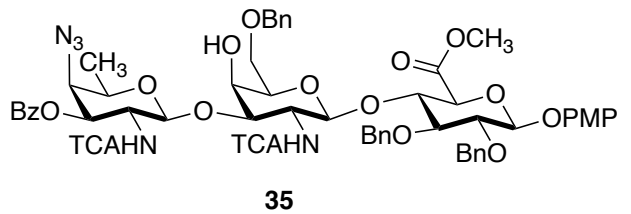

35

The trisaccharide 34 (1.40 g, $1.07 \mathrm{mmol}, 1$ equiv.) in $\mathrm{CH}_{2} \mathrm{Cl}_{2}(11 \mathrm{~mL})$ and $\mathrm{Et}_{3} \mathrm{SiH}(0.520 \mathrm{~mL}, 3.21$ mmol, 3.0 equiv.) was stirred with preactivated $4 \AA \mathrm{MS}(3.0 \mathrm{~g})$ for $30 \mathrm{~min}$ under an argon atmosphere. After cooling to $-78{ }^{\circ} \mathrm{C}$, the mixture was treated with $\mathrm{TfOH}(0.330 \mathrm{~mL}, 3.74 \mathrm{mmol}$, 3.5 equiv.). The mixture was maintained at that temperature for $2 \mathrm{~h}$. After TLC indicated complete consumption of the alcohol 34 starting material, $\mathrm{CH}_{3} \mathrm{OH}(3 \mathrm{~mL})$ and then $\mathrm{NEt}_{3}(1 \mathrm{~mL})$ were added in this order. The mixture was diluted with EtOAc $(50 \mathrm{~mL})$, washed with $\mathrm{H}_{2} \mathrm{O}(2 \times 50 \mathrm{~mL})$, dried over anhydrous $\mathrm{Na}_{2} \mathrm{SO}_{4}$, and concentrated in vacuo to yield the crude alcohol. Flash chromatography of the crude material eluting with EtOAc/Hexane (3:7) gave the alcohol 35 (1.20 $\mathrm{g}, 0.915 \mathrm{mmol}, 86 \%)$ as a white foam. The trisaccharide 35 had $\mathrm{R}_{\mathrm{f}}=0.30($ EtOAc/Hexane 1:1) visualized with ceric ammonium molybdate stain; $[\alpha] \mathrm{D}^{25}=-15.0^{\circ}\left(\mathrm{c}=0.002, \mathrm{CHCl}_{3}\right) ;{ }^{1} \mathrm{H} \mathrm{NMR}$ $\left(600 \mathrm{MHz}, \mathrm{CDCl}_{3}\right) \delta 8.05(\mathrm{dd}, J=8.4,1.4 \mathrm{~Hz}, 2 \mathrm{H}, \mathrm{Ar}), 7.63-7.53(\mathrm{~m}, 1 \mathrm{H}, \mathrm{Ar}), 7.50-7.42(\mathrm{~m}$, 2H, Ar), $7.41-7.36(\mathrm{~m}, 2 \mathrm{H}, \mathrm{Ar}), 7.36-7.23(\mathrm{~m}, 13 \mathrm{H}, \mathrm{Ar}), 7.13$ (d, J=7.5 Hz, 1H, NH), 7.01 (d, $J=8.7 \mathrm{~Hz}, 1 \mathrm{H}, \mathrm{NH}), 6.97(\mathrm{~d}, J=9.1 \mathrm{~Hz}, 2 \mathrm{H}, \mathrm{Ar}), 6.82$ (d, $J=9.1 \mathrm{~Hz}, 2 \mathrm{H}, \mathrm{Ar}), 5.42$ (dd, $J=11.1$, $3.5 \mathrm{~Hz}, 1 \mathrm{H}, \mathrm{H}-3 \mathrm{c}), 4.98$ (d, J=10.7 Hz, 1H, PhCHH), $4.95-4.89$ (m, 3H, PhCHH, H-1a, H-1b), $4.84(\mathrm{~d}, J=8.3 \mathrm{~Hz}, 1 \mathrm{H}, \mathrm{H}-1 \mathrm{c}), 4.78$ (d, $J=11.0 \mathrm{~Hz}, 1 \mathrm{H}, \mathrm{PhCH} H), 4.74(\mathrm{~d}, J=10.6 \mathrm{~Hz}, 1 \mathrm{H}$, $\mathrm{PhCH} H), 4.46(\mathrm{~d}, J=11.9 \mathrm{~Hz}, 1 \mathrm{H}, \mathrm{PhCHH}), 4.42-4.37$ (m, 1H, H-2c), 4.37 (d, $J=11.8 \mathrm{~Hz}, 1 \mathrm{H}$, $\mathrm{PhCH} H), 4.26$ (dd, $J=10.4,3.2 \mathrm{~Hz}, 1 \mathrm{H}, \mathrm{H}-3 \mathrm{a}), 4.19$ (ddd, $J=9.1,5.9,3.0 \mathrm{~Hz}, 1 \mathrm{H}, \mathrm{H}-4 \mathrm{~b}), 4.12$ (s, 1H, H-4a), 3.97-3.90 (m, 3H, H-2a, H-4c, H-5b), 3.85 - 3.79 (m, 1H, H-5c), 3.78 (s, 3H, OCH $)$, 
3.74 (s, 3H, $\left.\mathrm{CO}_{2} \mathrm{CH}_{3}\right), 3.72$ (dd, $\left.J=9.7,6.6 \mathrm{~Hz}, 1 \mathrm{H}, \mathrm{H}-6 \mathrm{a}\right), 3.68-3.61$ (m, 3H, H-2b, H-3b, H5a), 3.54 (dd, $J=9.7,5.5 \mathrm{~Hz}, 1 \mathrm{H}, \mathrm{H}-6 \mathrm{a}), 2.71(\mathrm{~s}, 1 \mathrm{H}, \mathrm{OH}), 1.40(\mathrm{~d}, J=6.3 \mathrm{~Hz}, 3 \mathrm{H}, \mathrm{H}-6 \mathrm{c}) ;{ }^{13} \mathrm{C}$ NMR (151 MHz, $\left.\mathrm{CDCl}_{3}\right) \delta 169.7\left(\mathrm{CO}_{2} \mathrm{CH}_{3}\right), 166.4(\mathrm{COPh}), 162.6(\mathrm{NHCO}), 162.2(\mathrm{NHCO})$, 155.6, 151.2, 138.6, 138.2, 138.1, 134.1, 130.3, 128.8, 128.5, 128.3, 128.3, 128.2, 127.9, 127.79, 127.62, 118.5, 114.7, 102.8 (C-1b GlcA), 99.7 (C-1c AAT), 98.9 (C-1a GalN), $92.9\left(C C_{3}\right), 92.3$ $\left(\mathrm{CCl}_{3}\right), 82.1(\mathrm{C}-3 \mathrm{~b}), 81.2(\mathrm{C}-2 \mathrm{~b}), 76.8(\mathrm{C}-4 \mathrm{~b}), 76.1(\mathrm{C}-3 \mathrm{a}), 75.9\left(\mathrm{PhCH}_{2}\right), 75.1\left(\mathrm{PhCH}_{2}\right), 74.2(\mathrm{C}-$ 5b), 73.7 (C-5a), $73.5\left(\mathrm{PhCH}_{2}\right), 72.8(\mathrm{C}-3 \mathrm{c}), 70.1$ (C-5c), $68.6\left(\mathrm{PhCH}_{2}\right), 67.9(\mathrm{C}-4 \mathrm{a}), 63.5(\mathrm{C}-4 \mathrm{c})$, $55.8\left(\mathrm{OCH}_{3}\right), 55.7(\mathrm{C}-2 \mathrm{a}), 53.2\left(\mathrm{CO}_{2} \mathrm{CH}_{3}\right), 53.1(\mathrm{C}-2 \mathrm{c}), 17.5$ (C-6c); HRMS (ESI) calcd. for $\mathrm{C}_{58} \mathrm{H}_{58} \mathrm{O}_{17} \mathrm{~N}_{5} \mathrm{Cl}_{6}(\mathrm{M}-\mathrm{H})^{-}$1306.1964, found $1306.1930 \mathrm{~m} / \mathrm{z}$. 
p-Methoxyphenyl (3-O-benzoyl-4-azido-2,4,6-trideoxy-2-trichloroacetamido- $\beta$-Dgalactopyranosyl)-( $\rightarrow 3)-(4-(\mathrm{N}-$ benzyloxycarbonyl)aminoethyl-phos-phonato-6- $O$-benzyl-2deoxy-2-trichloroacetamido- $\beta$-D-galactopyranosyl)-(1 $\rightarrow 4)$-(methyl 2,3-di- $O$-benzyl- $\beta$-Dglucopyranosid)uronate (36):
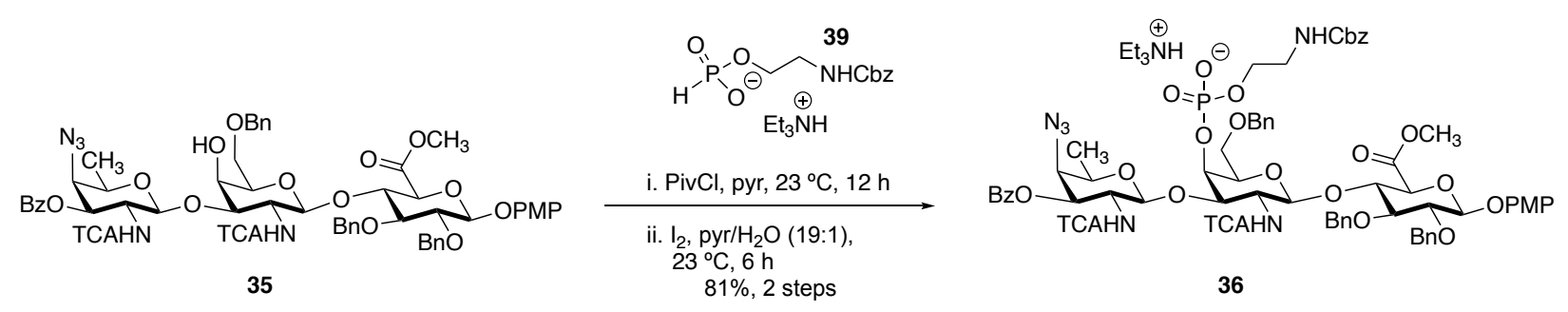

Ethanolamine H-phosphonate $\mathbf{3 9}^{8,9}$ (208 mg, $0.58 \mathrm{mmol}, 2.0$ equiv.) and trisaccharide 35 (108 mg, $0.082 \mathrm{mmol}, 1.0$ equiv. $)$ were co-evaporated with anhydrous pyridine $(3 \times 5 \mathrm{~mL})$ in two separate portions of $10 \mathrm{~mL}$ of $\mathrm{RBF}$ and dried under high vacuum overnight. The ethanolamine $\mathrm{H}-$ phosphonate 39 was dissolved in anhydrous pyridine $(1 \mathrm{~mL})$ followed by the addition of pivaloyl chloride $(0.070 \mathrm{~mL}, 0.58 \mathrm{mmol}, 2.0$ equiv.) stirring for $15 \mathrm{~min}$ at room temperature under an argon atmosphere. To that mixture, a solution of trisaccharide $\mathbf{3 5}$ in anhydrous pyridine $(1.5 \mathrm{~mL})$ was added, and the resulting mixture was stirred at room temperature for $12 \mathrm{~h}$. Iodine (158 $\mathrm{mg}, 0.62$ mmol, 2.5 equiv.) in a mixture of pyridine and $\mathrm{H}_{2} \mathrm{O}(19: 1,0.5 \mathrm{~mL})$ was added to oxidize $\mathrm{P}(\mathrm{III})$ to $\mathrm{P}(\mathrm{V})$ and stirred for additional $6 \mathrm{~h}$ at room temperature. The reaction mixture was diluted with $\mathrm{CH}_{2} \mathrm{Cl}_{2}(50 \mathrm{~mL})$ and the organic layer washed with satd. aq. $\mathrm{Na}_{2} \mathrm{~S}_{2} \mathrm{O}_{3}(20 \mathrm{~mL} \times 1)$, followed by 1M TEAB (triethylammonium bicarbonate) $(20 \mathrm{~mL}$ x 1) buffer solution, and dried over anhydrous $\mathrm{Na}_{2} \mathrm{SO}_{4}$. Solvents were concentrated in vacuo, and the crude was purified by flash column chromatography with $\mathrm{E}_{\mathrm{t}_{3}} \mathrm{~N}$ deactivated silica gel to give $36(118 \mathrm{mg}, 0.082 \mathrm{mmol}, 86 \%)$ as a white sticky liquid. Phosphorated 36 had $\mathrm{R}_{\mathrm{f}}=0.5\left(\mathrm{CH}_{2} \mathrm{Cl}_{2} / \mathrm{CH}_{3} \mathrm{OH}=9: 1\right)$ visualized with ceric ammonium molybdate stain; $[\alpha] \mathrm{D}^{25}=-5.7^{\circ}\left(\mathrm{c}=0.0017, \mathrm{CHCl}_{3}\right) ;{ }^{1} \mathrm{H} \mathrm{NMR}\left(600 \mathrm{MHz}, \mathrm{CDCl}_{3}\right) \delta$ $8.05(\mathrm{~d}, J=7.7 \mathrm{~Hz}, 2 \mathrm{H}, \mathrm{Ar}), 7.55(\mathrm{t}, J=7.4 \mathrm{~Hz}, 1 \mathrm{H}, \mathrm{Ar}), 7.49-7.20$ (m, 22H, Ar), 7.01 (d, $J=$ 
$8.7 \mathrm{~Hz}, 1 \mathrm{H}, \mathrm{NH}), 6.97$ (d, $J=9.0 \mathrm{~Hz}, 2 \mathrm{H}, \mathrm{Ar}), 6.81(\mathrm{~d}, J=9.1 \mathrm{~Hz}, 2 \mathrm{H}, \mathrm{Ar}), 6.50(\mathrm{~d}, J=5.0 \mathrm{~Hz}$, 1H, NH), 6.13 (d, $J=9.7 \mathrm{~Hz}, 1 \mathrm{H}, \mathrm{H}-4 \mathrm{a}), 5.32$ (d, $J=8.1 \mathrm{~Hz}, 1 \mathrm{H}, \mathrm{H}-1 \mathrm{c}), 5.13-4.98$ (m, 2H, $\mathrm{PhCHH}, \mathrm{PhCH}_{2}$ ), 4.96 (d, $\left.J=6.8 \mathrm{~Hz}, 1 \mathrm{H}, \mathrm{H}-1 \mathrm{~b}\right), 4.90-4.83$ (m, 2H, H-3c, $\mathrm{PhCH} H$ ), 4.80 (d, $J=$ $8.2 \mathrm{~Hz}, 1 \mathrm{H}, \mathrm{H}-1 \mathrm{a}), 4.74$ (d, $J=11.1 \mathrm{~Hz}, 1 \mathrm{H}, \mathrm{PhCHH}), 4.66$ (d, $J=10.8 \mathrm{~Hz}, 1 \mathrm{H}, \mathrm{PhCH} H), 4.50$ (d, $J=11.8 \mathrm{~Hz}, 1 \mathrm{H}, \mathrm{PhCHH}), 4.43(\mathrm{~d}, J=12.0 \mathrm{~Hz}, 1 \mathrm{H}, \mathrm{PhCH} H), 4.36$ (d, $J=8.2 \mathrm{~Hz}, 1 \mathrm{H}, \mathrm{H}-5 \mathrm{c}), 4.28$ (t, $J=8.4 \mathrm{~Hz}, 1 \mathrm{H}, \mathrm{H}-4 \mathrm{~b}), 4.02-3.95$ (m, 4H, H-3a, H-5b, H-2c, H-4c), $3.93-3.80$ (m, 4H, H-2a, H-6a, PEtN-CH2), 3.77 (s, 3H, OCH $\left.H_{3}\right), 3.74-3.73(\mathrm{~m}, 1 \mathrm{H}, \mathrm{H}-5 \mathrm{a}), 3.72\left(\mathrm{~s}, 3 \mathrm{H}, \mathrm{CO}_{2} \mathrm{CH}_{3}\right), 3.70-$ 3.58 (m, 3H, H-2b, H-3b, H-6a), 3.41 - 3.32 (m, 2H, PEtN-CH $H_{2}$ ), 1.38 (d, $J=6.3 \mathrm{~Hz}, 3 \mathrm{H}, \mathrm{H}-6 \mathrm{c}$ ); ${ }^{13} \mathrm{C} \mathrm{NMR}\left(151 \mathrm{MHz}, \mathrm{CDCl}_{3}\right) \delta 169.4\left(\mathrm{CO}_{2} \mathrm{CH}_{3}\right), 165.9(\mathrm{COPh}), 163.5(\mathrm{NHCO}), 162.5(\mathrm{NHCO})$, $156.6,155.5,151.2,138.8,138.4,138.2,136.8,133.6,130.2,128.7,128.6,128.5,128.4,128.4$, 128.4, 128.3, 128.1, 128.1, 127.7, 127.7, 127.6, 127.5, 118.36, 114.66, 102.4 (C-1b GlcA), 100.7 (C-1a GalN), 96.5 (C-1c AAT), $92.9\left(\mathrm{CCl}_{3}\right), 92.7\left(\mathrm{CCl}_{3}\right), 82.1$ (C-3b), $81.0(\mathrm{C}-2 \mathrm{~b}), 77.0(\mathrm{C}-4 \mathrm{~b})$, $75.3\left(\mathrm{PhCH}_{2}\right), 74.9\left(\mathrm{PhCH}_{2}\right), 74.8$ (C-5a), $74.4(\mathrm{C}-5 \mathrm{~b}), 73.8\left(\mathrm{PhCH}_{2}\right), 73.5(\mathrm{C}-3 \mathrm{c}), 70.7(\mathrm{C}-5 \mathrm{c})$, 70.3 (C-4a), 69.7 (C-6a), $69.5(\mathrm{C}-3 \mathrm{a}), 66.6\left(\mathrm{PhCH}_{2}\right), 64.3\left(\mathrm{PEtN}^{-} \mathrm{CH}_{2}\right), 63.3(\mathrm{C}-4 \mathrm{c}), 55.7\left(\mathrm{OCH}_{3}\right)$, 54.1 (C-2a), 53.9 (C-2c), $52.8\left(\mathrm{CO}_{2} \mathrm{CH}_{3}\right), 42.6\left(\mathrm{PEtN}-\mathrm{CH}_{2}\right), 17.9$ (C-6c); ${ }^{31} \mathrm{P}$ NMR (162 MHz,

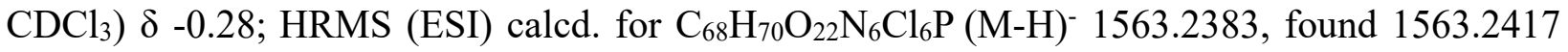
$m / z$. 
p-Methoxyphenyl 2-acetamido-4-amino-2,4,6-trideoxy- $\beta$-D-galactopyranosyl-(1 $\rightarrow 3)-4-O$ aminoethyl-phospho-nato- $\beta$-D-galactopyranosyl-( $1 \rightarrow 4)-O$ - $\beta$-D-Glucopyranosyluronic acid (29):
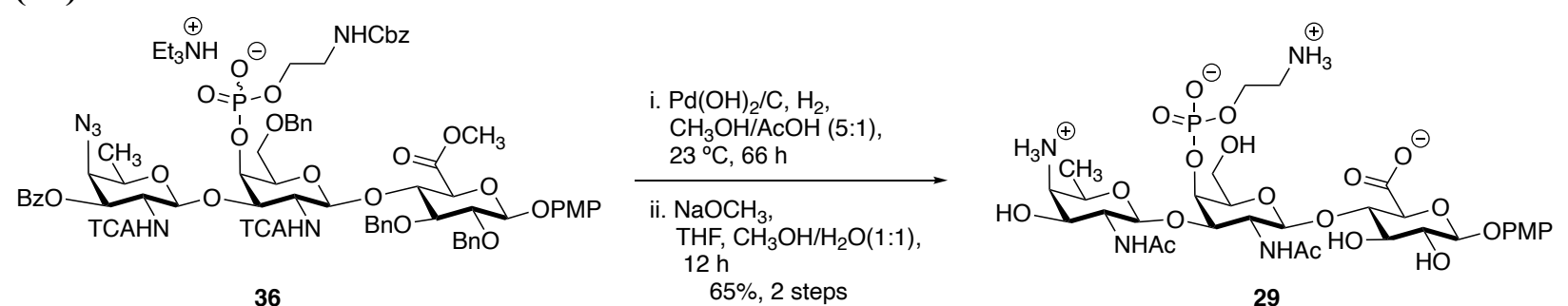

Protected trisaccharide 36 (36 mg, $0.022 \mathrm{mmol}, 1.0$ equiv.) was placed under argon. To this flask was wet $\mathrm{Pd}(\mathrm{OH})_{2} / \mathrm{C}(20 \% \mathrm{Pd}$ by weight, $110 \mathrm{mg}, 0.15 \mathrm{mmol}, 7.0$ equiv. $) . \mathrm{CH}_{3} \mathrm{OH}(5 \mathrm{~mL})$ and $\mathrm{AcOH}(1 \mathrm{~mL})$ was added to this mixture, and then, $\mathrm{H}_{2}$ gas was bubbled through the solution at 23 ${ }^{\circ} \mathrm{C}$ for 30 min. ${ }^{10}$ After the $\mathrm{H}_{2}$ atmosphere was established, the reaction was stirred for $66 \mathrm{~h}$ (progress monitored by MS). The mixture was then, filtered over Celite and concentrated in vacuo to yield the crude product. The crude material was dissolved in THF $(3 \mathrm{~mL})$ at $23{ }^{\circ} \mathrm{C}$ and then, treated dropwise, with a $1.5 \mathrm{M} \mathrm{NaOCH}_{3}$ solution in $1: 1 \mathrm{CH}_{3} \mathrm{OH} / \mathrm{H}_{2} \mathrm{O}(5.0 \mathrm{~mL}, 1.95 \mathrm{mmol})$. After stirring for $12 \mathrm{~h}$ (progress monitored by MS), AcOH was added until neutral $\mathrm{pH}$ was achieved. The crude material was concentrated in vacuo and was purified by size exclusion chromatography (Bio-Gel P2 gel) using deionized $\mathrm{H}_{2} \mathrm{O}$ as an eluant. Fractions containing the desired product (determined from MS) were combined and lyophilized to $29(12.0 \mathrm{mg}, 0.015 \mathrm{mmol}, 68 \%)$ as a white powder; $[\alpha] \mathrm{D}^{25}=-29.1^{\circ}\left(\mathrm{c}=0.003, \mathrm{H}_{2} \mathrm{O}\right) ;{ }^{1} \mathrm{H} \mathrm{NMR}\left(600 \mathrm{MHz}, \mathrm{D}_{2} \mathrm{O}\right) \delta 7.11(\mathrm{~d}, J=9.1 \mathrm{~Hz}$, 2H), $6.99(\mathrm{~d}, J=9.1 \mathrm{~Hz}, 2 \mathrm{H}), 5.01(\mathrm{~d}, J=7.9 \mathrm{~Hz}, 1 \mathrm{H}), 4.72(\mathrm{dd}, J=9.2,2.8 \mathrm{~Hz}, 1 \mathrm{H}), 4.60(\mathrm{~d}, J=$ $8.4 \mathrm{~Hz}, 1 \mathrm{H}), 4.54(\mathrm{~d}, J=8.4 \mathrm{~Hz}, 1 \mathrm{H}), 4.24-4.15(\mathrm{~m}, 2 \mathrm{H}), 4.09-4.00(\mathrm{~m}, 2 \mathrm{H}), 3.97(\mathrm{q}, J=6.0$ $\mathrm{Hz}, 1 \mathrm{H}), 3.91-3.83(\mathrm{~m}, 4 \mathrm{H}), 3.82(\mathrm{~s}, 3 \mathrm{H}), 3.81-3.76(\mathrm{~m}, 3 \mathrm{H}), 3.71(\mathrm{t}, J=8.7 \mathrm{~Hz}, 1 \mathrm{H}), 3.62(\mathrm{dd}$, $J=9.5,7.9 \mathrm{~Hz}, 1 \mathrm{H}), 3.55(\mathrm{~d}, J=4.7 \mathrm{~Hz}, 1 \mathrm{H}), 3.30(\mathrm{t}, J=5.5 \mathrm{~Hz}, 2 \mathrm{H}), 2.10(\mathrm{~s}, 3 \mathrm{H}), 2.02(\mathrm{~s}, 3 \mathrm{H})$, $1.34(\mathrm{~d}, J=6.6 \mathrm{~Hz}, 3 \mathrm{H}) ;{ }^{13} \mathrm{C} \mathrm{NMR}\left(151 \mathrm{MHz}, \mathrm{D}_{2} \mathrm{O}\right) \delta 175.3,175.1,155.8,152.0,119.2,116.1$, $103.9,102.3,102.1,80.9,79.2,77.7,75.9,75.3,74.8,73.6,69.0,68.4,63.2,62.2,56.8,55.6,53.1$, 
52.0, 41.3, 23.7, 23.4, 16.8; ${ }^{31} \mathrm{P}$ NMR $\left(162 \mathrm{MHz}, \mathrm{D}_{2} \mathrm{O}\right) \delta-0.30$; HRMS (ESI) calcd. for $\mathrm{C}_{31} \mathrm{H}_{48} \mathrm{O}_{19} \mathrm{~N}_{4} \mathrm{P}(\mathrm{M}-\mathrm{H})^{-} 811.2656$, found $811.2631 \mathrm{~m} / \mathrm{z}$. Our spectroscopic data strongly matched previously reported data. ${ }^{11}$

O:Xcaliburldatal2021MMay\JMN-E-1052
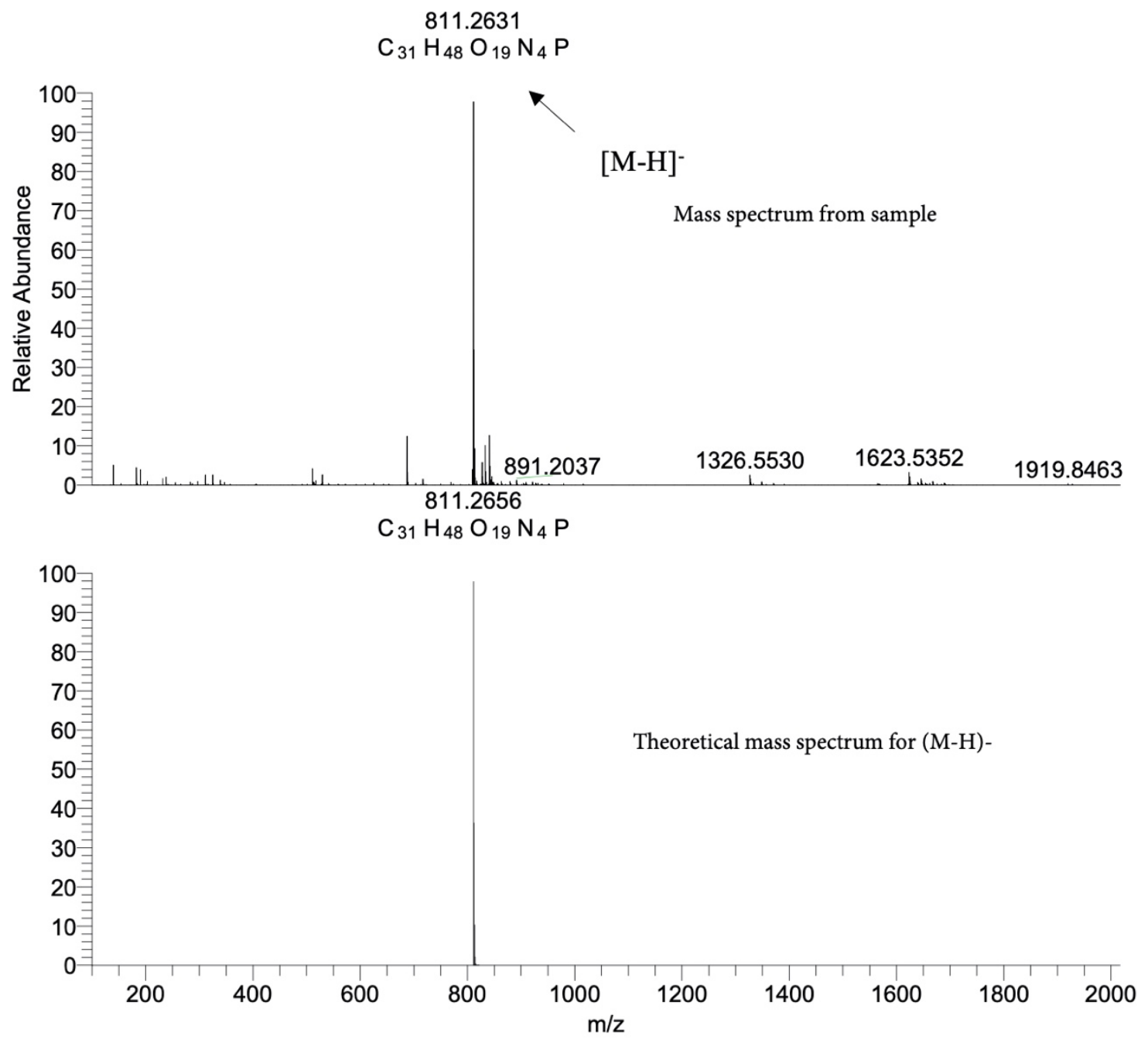
Table S1. Comparison of ${ }^{1} \mathrm{H}$ and ${ }^{13} \mathrm{C}$ NMR Chemical Shifts (ppm) between isolated polysaccharide and synthetic 29

\begin{tabular}{|c|c|c|c|c|c|c|c|c|c|}
\hline \multirow{2}{*}{\multicolumn{2}{|c|}{ Residue }} & \multicolumn{8}{|c|}{ Chemical shifts (ppm) } \\
\hline & & $\mathrm{C} 1 / \mathrm{H} 1$ & $\mathrm{C} 2 / \mathrm{H} 2$ & $\mathrm{C} 3 / \mathrm{H} 3$ & $\mathrm{C} 4 / \mathrm{H} 4$ & $\mathrm{C} 5 / \mathrm{H} 5$ & C6/H6 & NAc & $\mathrm{C}=\mathrm{O}$ \\
\hline \multirow{4}{*}{$\rightarrow 3)-\beta-\mathrm{D}$ Fuc $p$ NAc $4 \mathrm{~N}-(1 \rightarrow$} & \multirow{2}{*}{ isolated $^{\mathrm{a}}$} & 103.8 & 51.9 & 78.8 & 55.5 & 68.3 & 16.8 & 23.5 & \multirow{2}{*}{$\begin{array}{r}175.6- \\
176.0\end{array}$} \\
\hline & & 4.56 & 3.87 & 4.11 & 3.77 & 3.94 & 1.30 & 2.01 & \\
\hline & \multirow{2}{*}{ synthetic } & 103.9 & 53.1 & 69.0 & 55.7 & 68.4 & 16.8 & 23.5 & \multirow{2}{*}{$\begin{array}{r}175.4- \\
175.3 \\
\end{array}$} \\
\hline & & 4.54 & 3.79 & 4.02 & 3.54 & 3.97 & 1.34 & 2.02 & \\
\hline \multirow{4}{*}{$\rightarrow 3)-\beta-\mathrm{D}-\mathrm{Gal} p \mathrm{NAc}-(1 \rightarrow$} & \multirow{2}{*}{ isolated $^{\mathrm{a}}$} & 102.2 & 52.1 & 79.3 & 75.4 & 76.1 & 62.3 & 23.8 & \multirow{2}{*}{$\begin{array}{c}175.6 \\
176.0\end{array}$} \\
\hline & & 4.53 & 4.00 & 3.82 & 4.68 & 3.76 & $3.79,3.75$ & 2.04 & \\
\hline & \multirow{2}{*}{ synthetic } & 102.1 & 52.0 & 79.2 & 75.3 & 75.9 & 62.2 & 23.7 & \multirow{2}{*}{$\begin{array}{r}175.4- \\
175.3\end{array}$} \\
\hline & & 4.60 & 4.04 & 3.86 & 4.72 & 3.80 & $3.83,3.78$ & 2.10 & \\
\hline \multirow{4}{*}{$\rightarrow 4)-\beta-\mathrm{D}-\mathrm{Glc} p \mathrm{~A}-(1 \rightarrow$} & \multirow{2}{*}{ isolated $^{\mathrm{a}}$} & 105.6 & 73.4 & 74.9 & 81.1 & 77.5 & 175.6 & & \\
\hline & & 4.51 & 3.36 & 3.58 & 3.74 & 3.73 & & & \\
\hline & \multirow{2}{*}{ synthetic } & 102.3 & 73.6 & 74.8 & 80.9 & 77.8 & 175.1 & & \\
\hline & & 5.01 & 3.62 & 3.71 & 3.86 & 3.85 & & & \\
\hline \multirow{4}{*}{ PEtN } & \multirow{2}{*}{ isolated $^{\mathrm{a}}$} & 63.3 & 41.4 & & & & & & \\
\hline & & 4.16 & 3.27 & & & & & & \\
\hline & \multirow{2}{*}{ synthetic } & 63.3 & 41.3 & & & & & & \\
\hline & & 4.20 & 3.30 & & & & & & \\
\hline
\end{tabular}

${ }^{a}$ Data from isolation ref: Carbohydrate Res. 2015, 1-4. 
${ }^{1} \mathrm{H}$ NMR (600 MHz, MeOD) of Compound 10

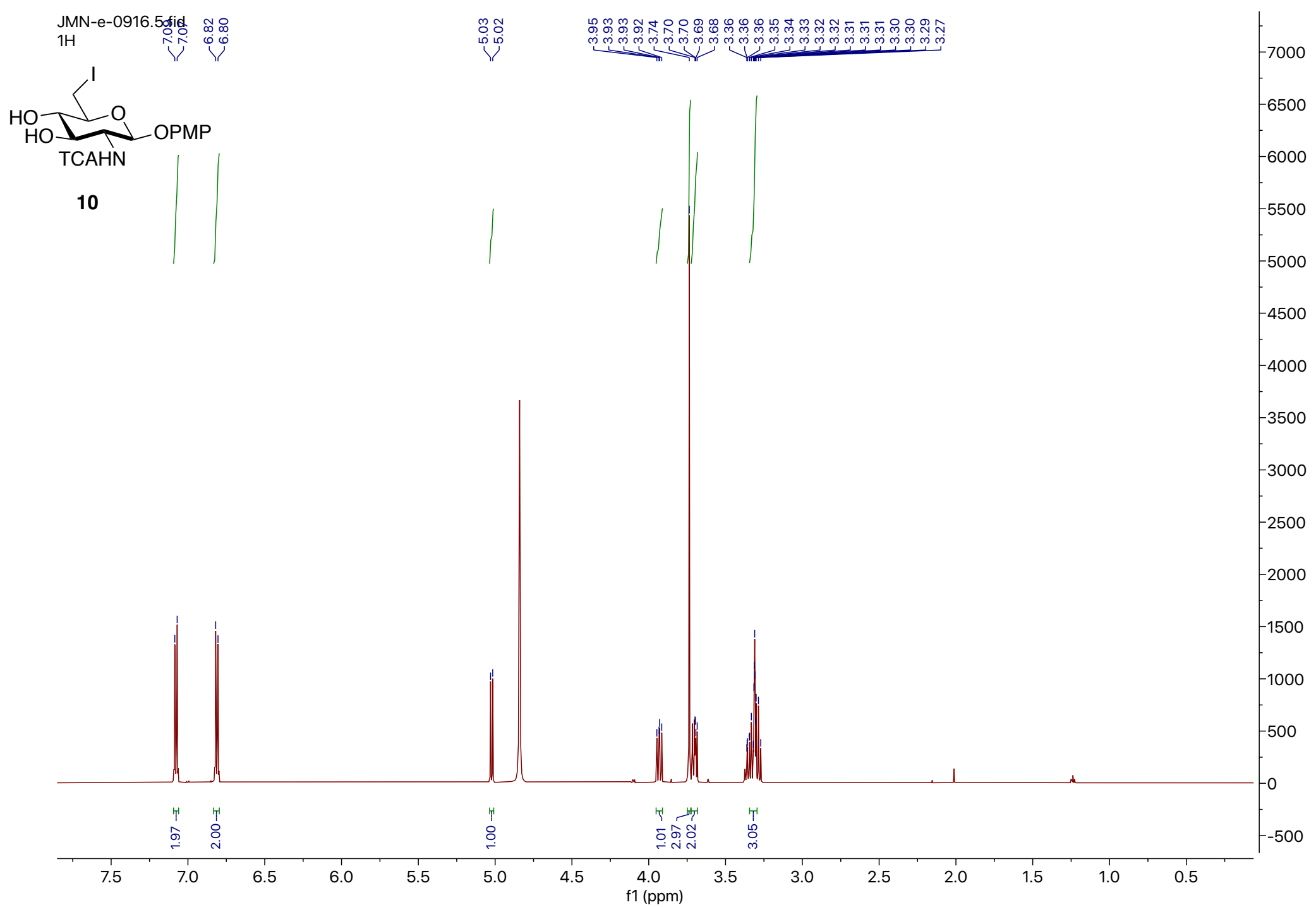


${ }^{13}$ C NMR (151 MHz, MeOD) of Compound 10

JMN-e-0916.8.fid

$13 \mathrm{C}$

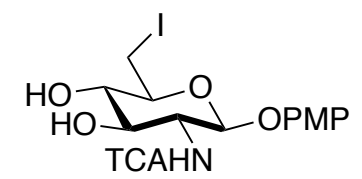

10 ict

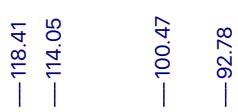

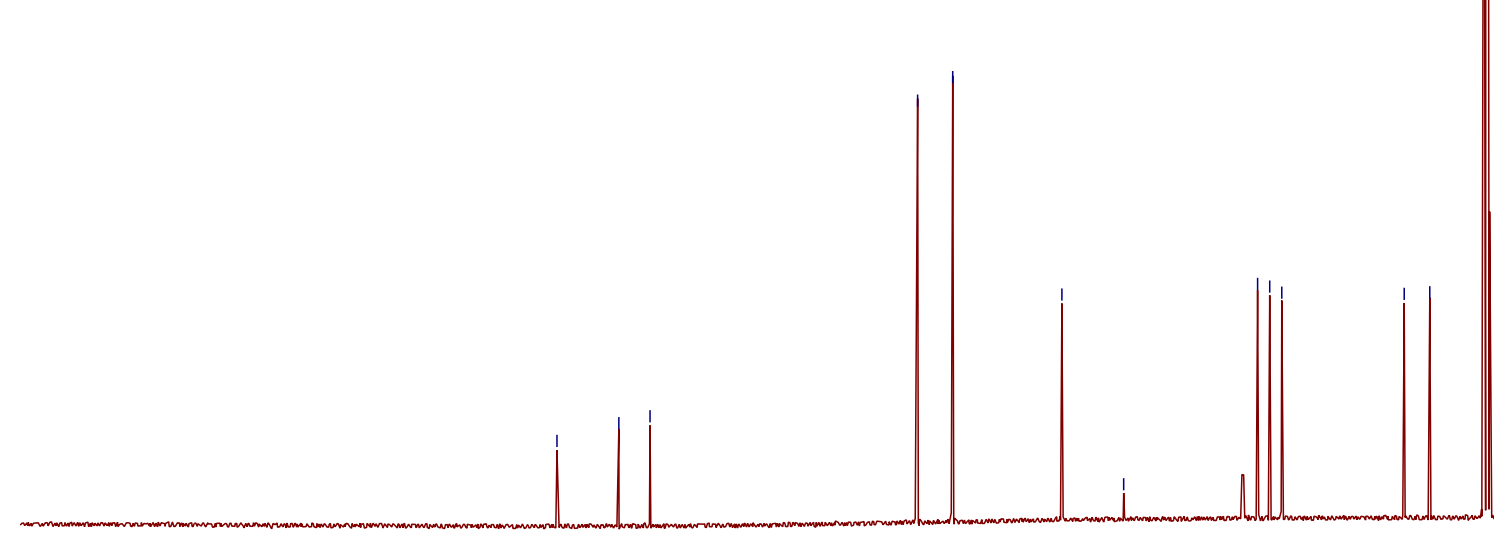

14000 
${ }^{1} \mathrm{H}-{ }^{13} \mathrm{C}$ HSQC (MeOD) of Compound 10

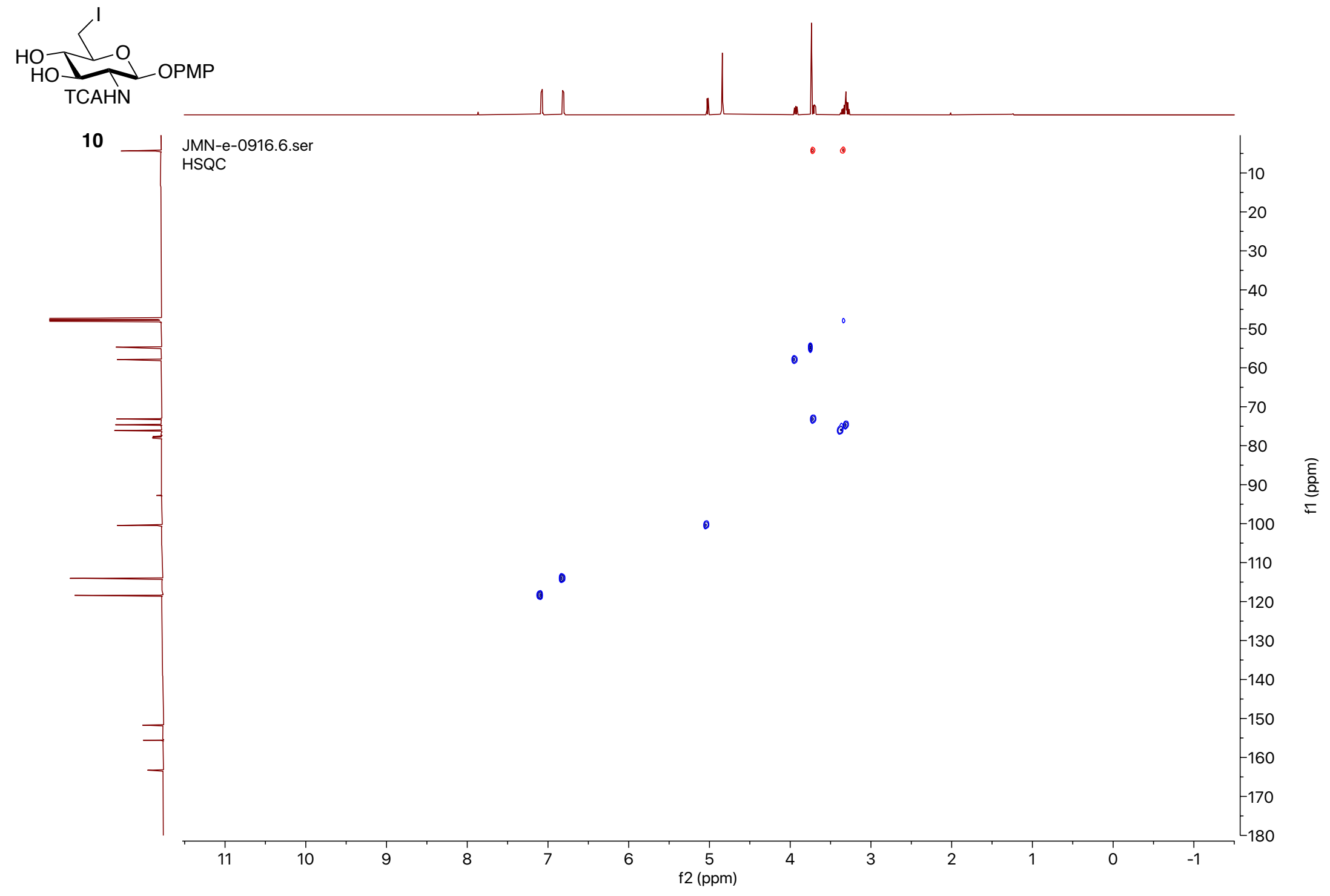


${ }^{1} \mathrm{H}-{ }^{1} \mathrm{H}$ COSY (MeOD) of Compound 10

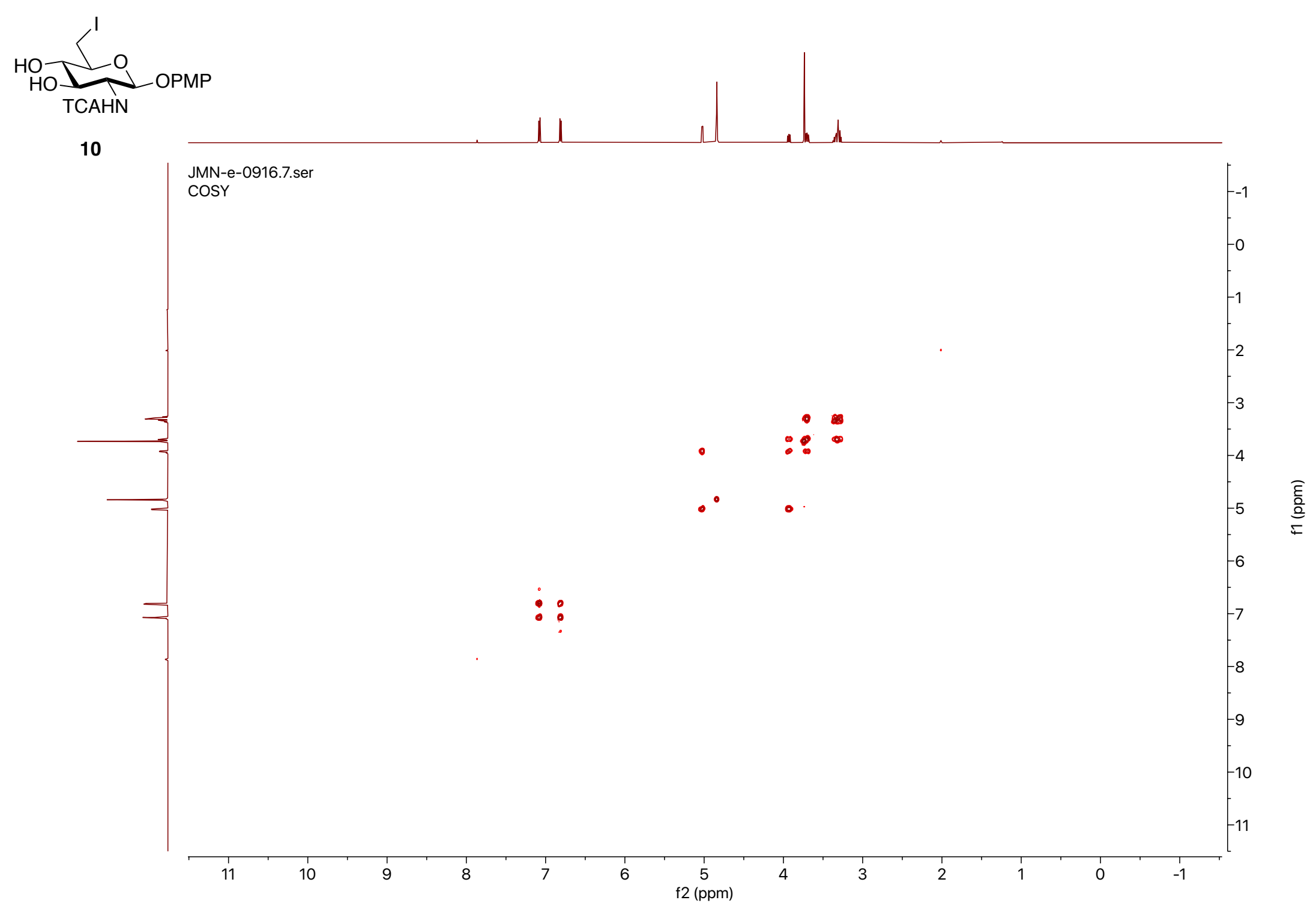


${ }^{1}$ H NMR (600 MHz, MeOD) of Compound 11

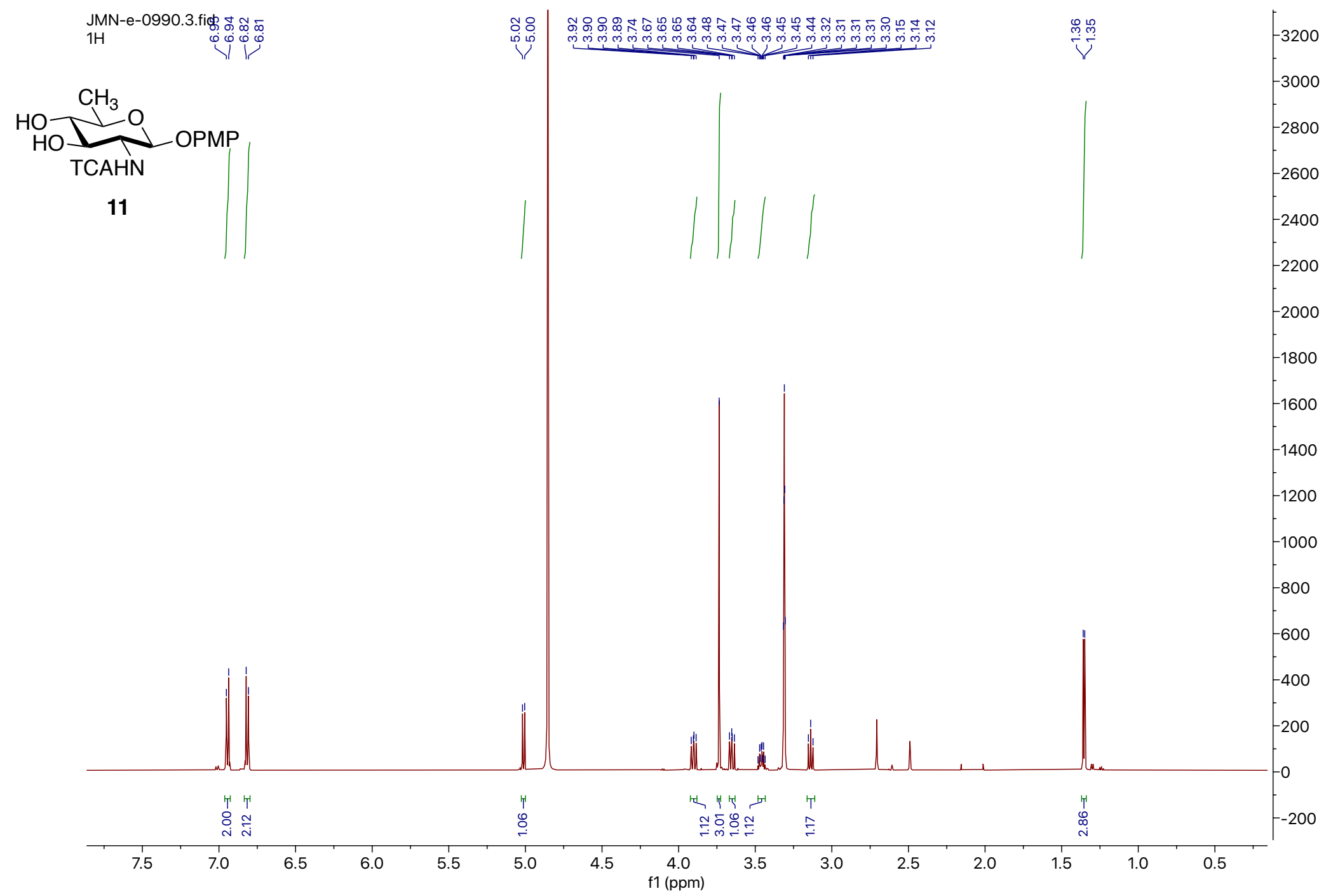


${ }^{13}$ C NMR (151 MHz, MeOD) of Compound 11

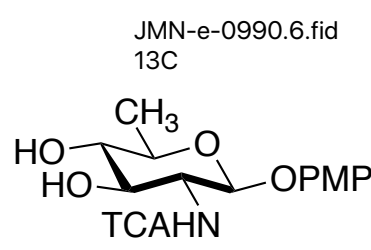

11 il

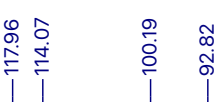

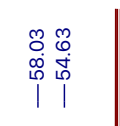

$-10000$

$-9000$

$-8000$

$-7000$

$-6000$

$-5000$

$-4000$

$-3000$

$-2000$

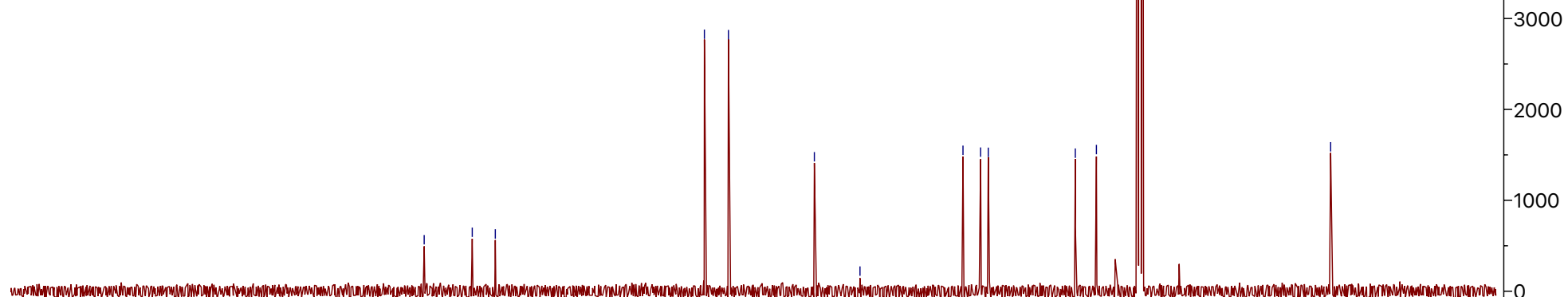

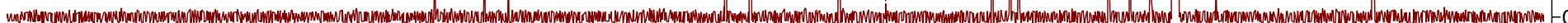


${ }^{1} \mathrm{H}-{ }^{13} \mathrm{C}$ HSQC (MeOD) of Compound 11

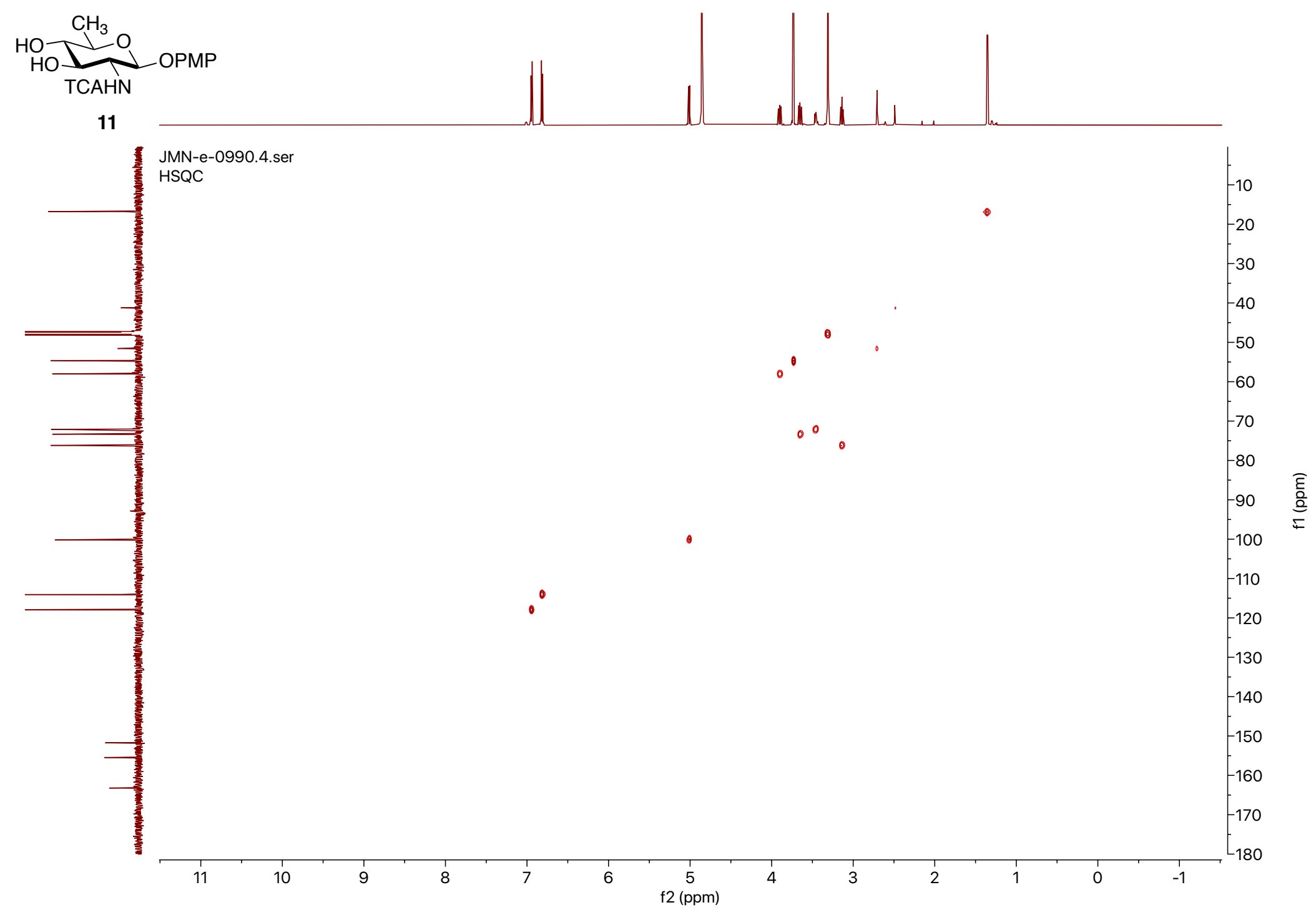


${ }^{1} \mathrm{H}-{ }^{1} \mathrm{H}$ COSY (MeOD) of Compound 11

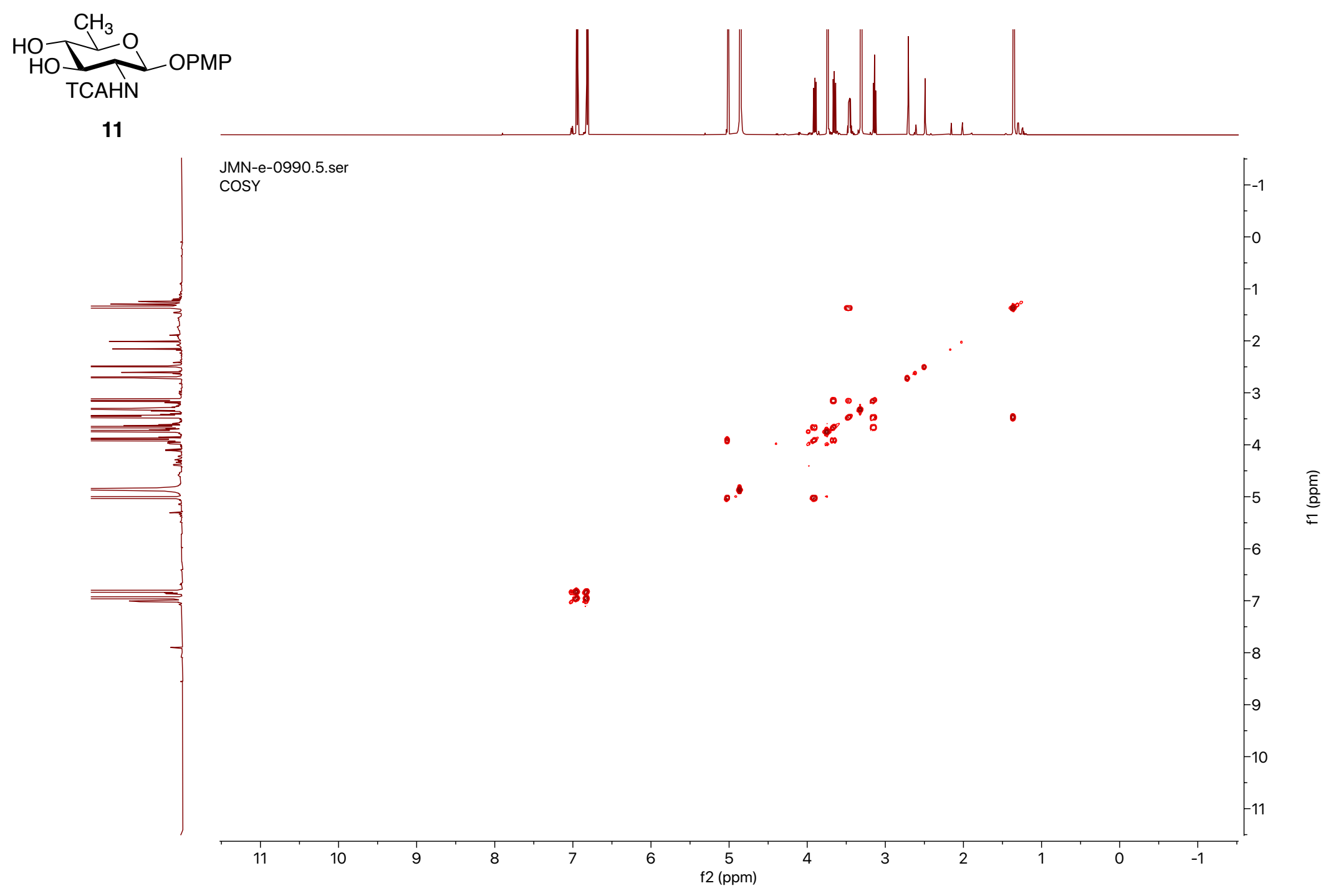


${ }^{1} \mathrm{H}$ NMR (600 MHz, $\mathrm{CDCl}_{3}$ ) of Compound 12

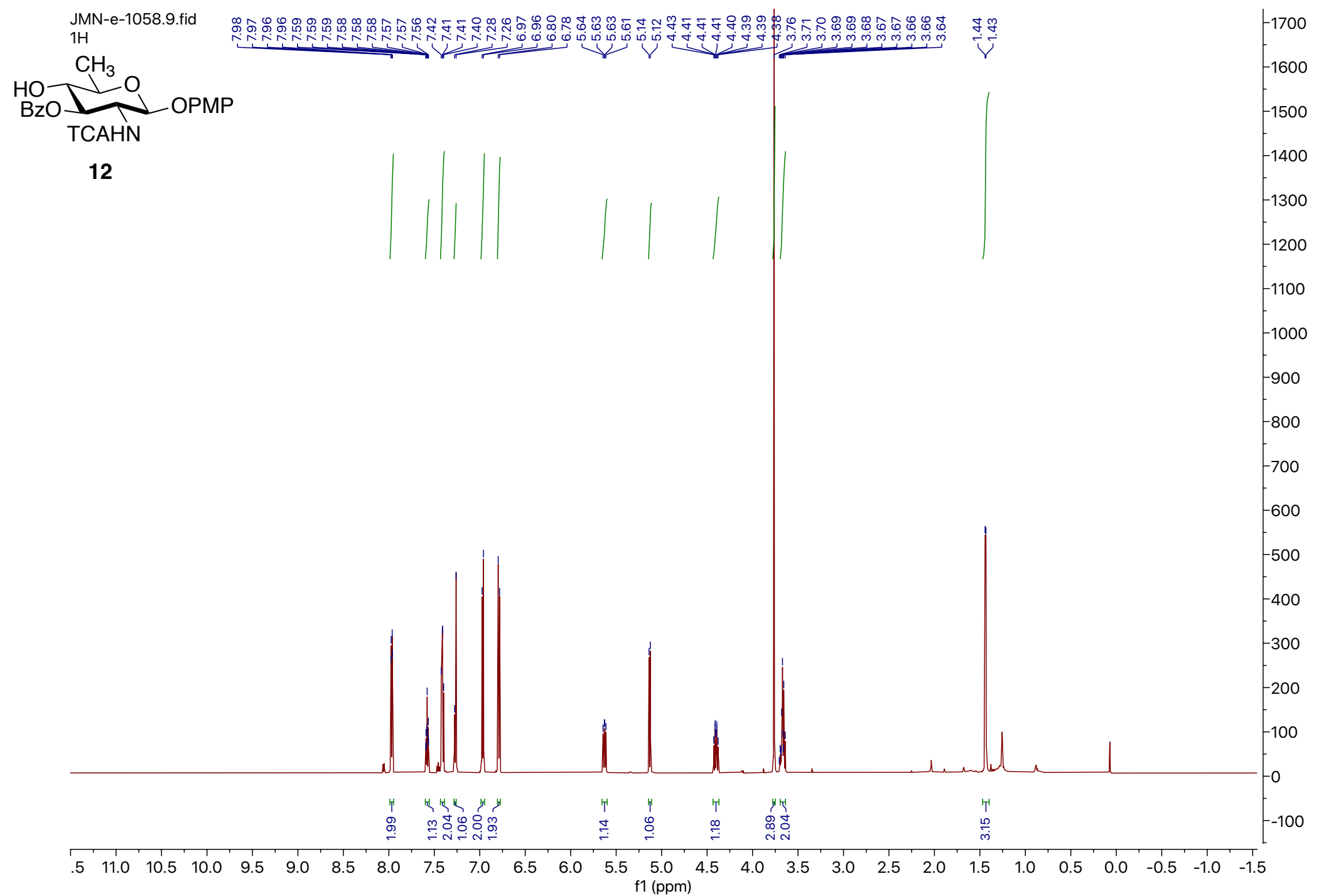


${ }^{13} \mathrm{C}$ NMR (151 MHz, $\left.\mathrm{CDCl}_{3}\right)$ of Compound 12

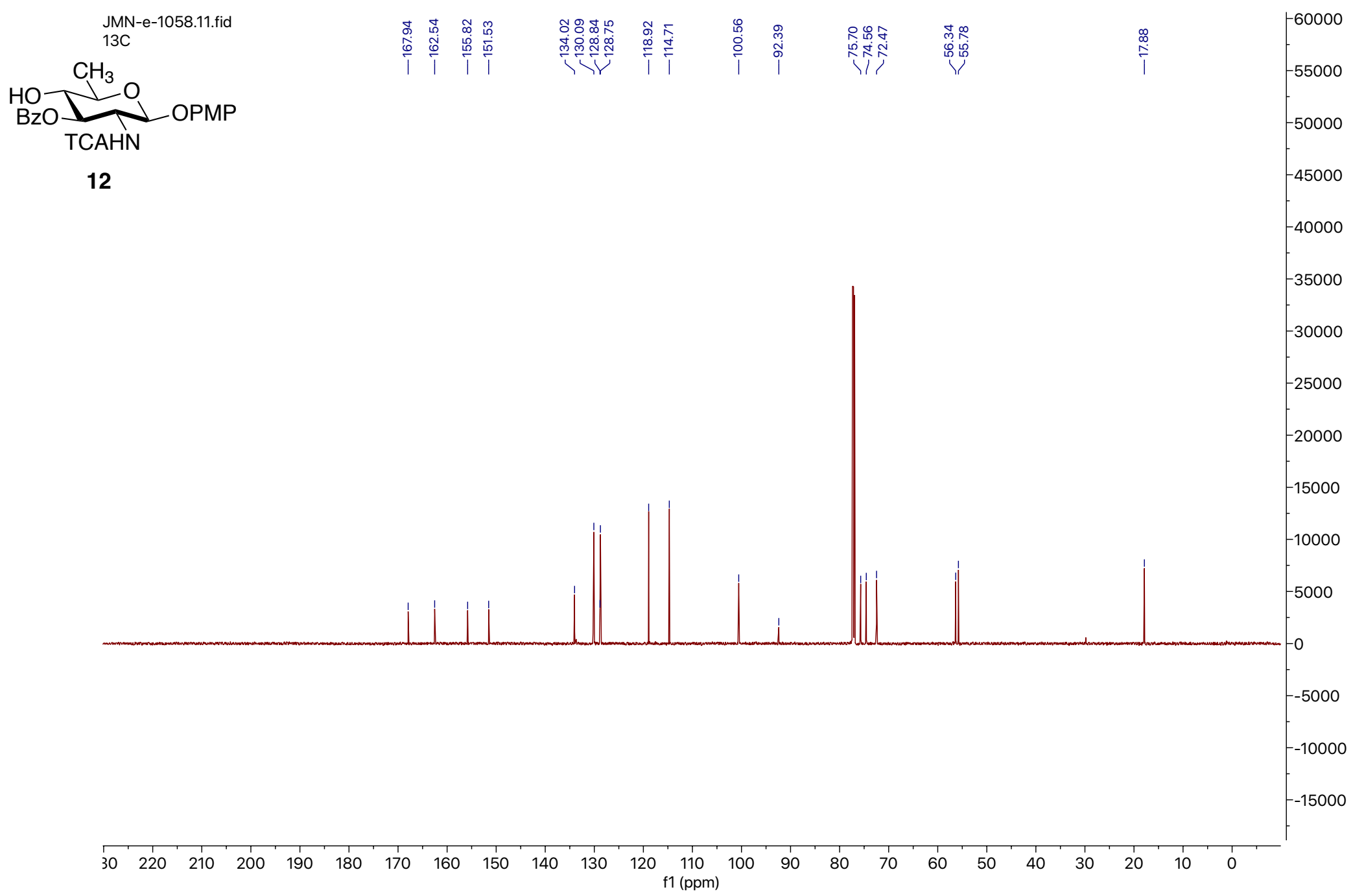


${ }^{1} \mathrm{H}-{ }^{13} \mathrm{C}$ HSQC $\left(\mathrm{CDCl}_{3}\right)$ of Compound 12

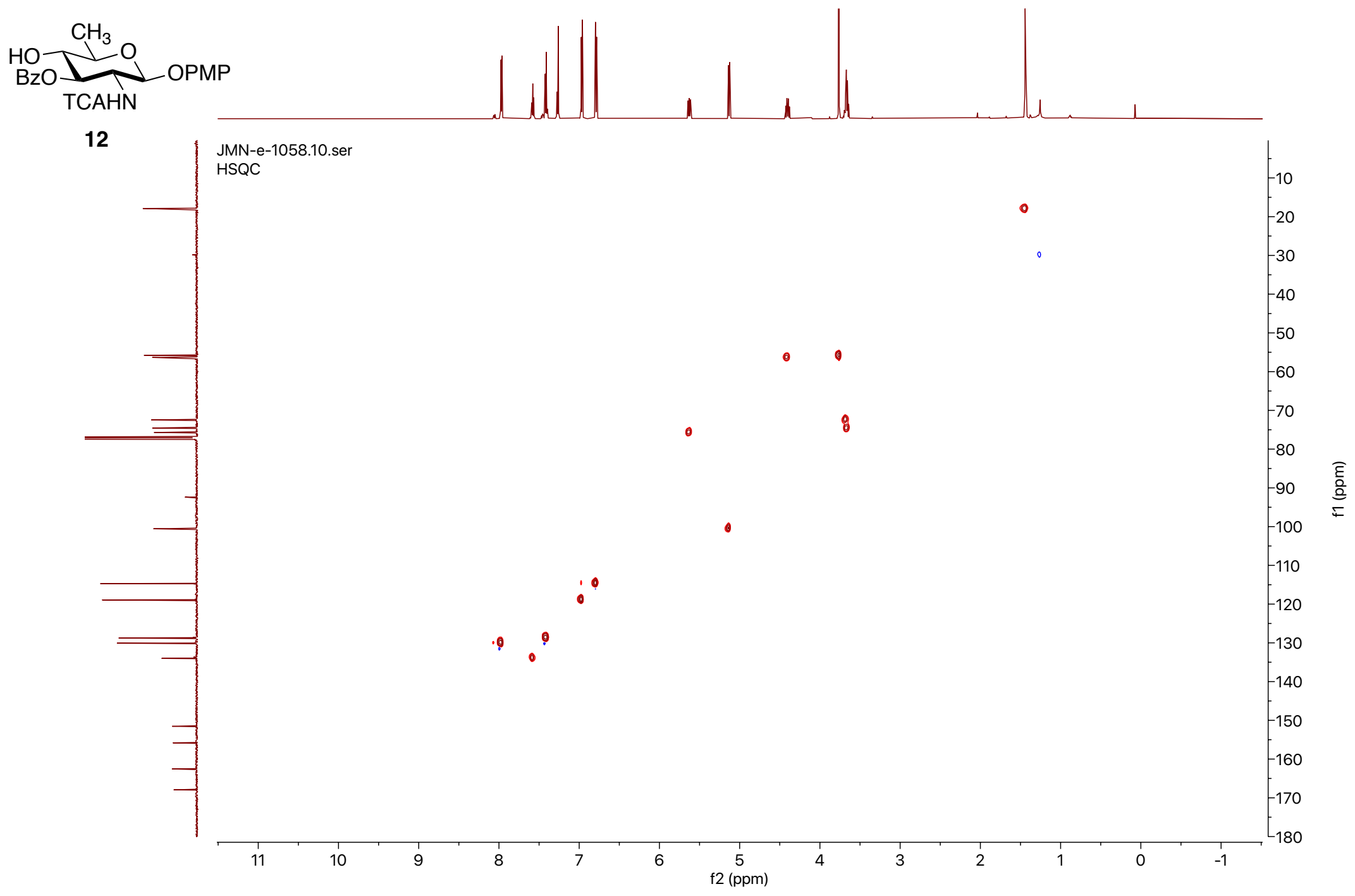


${ }^{1} \mathrm{H}-{ }^{1} \mathrm{H}$ COSY $\left(\mathrm{CDCl}_{3}\right)$ of Compound 12

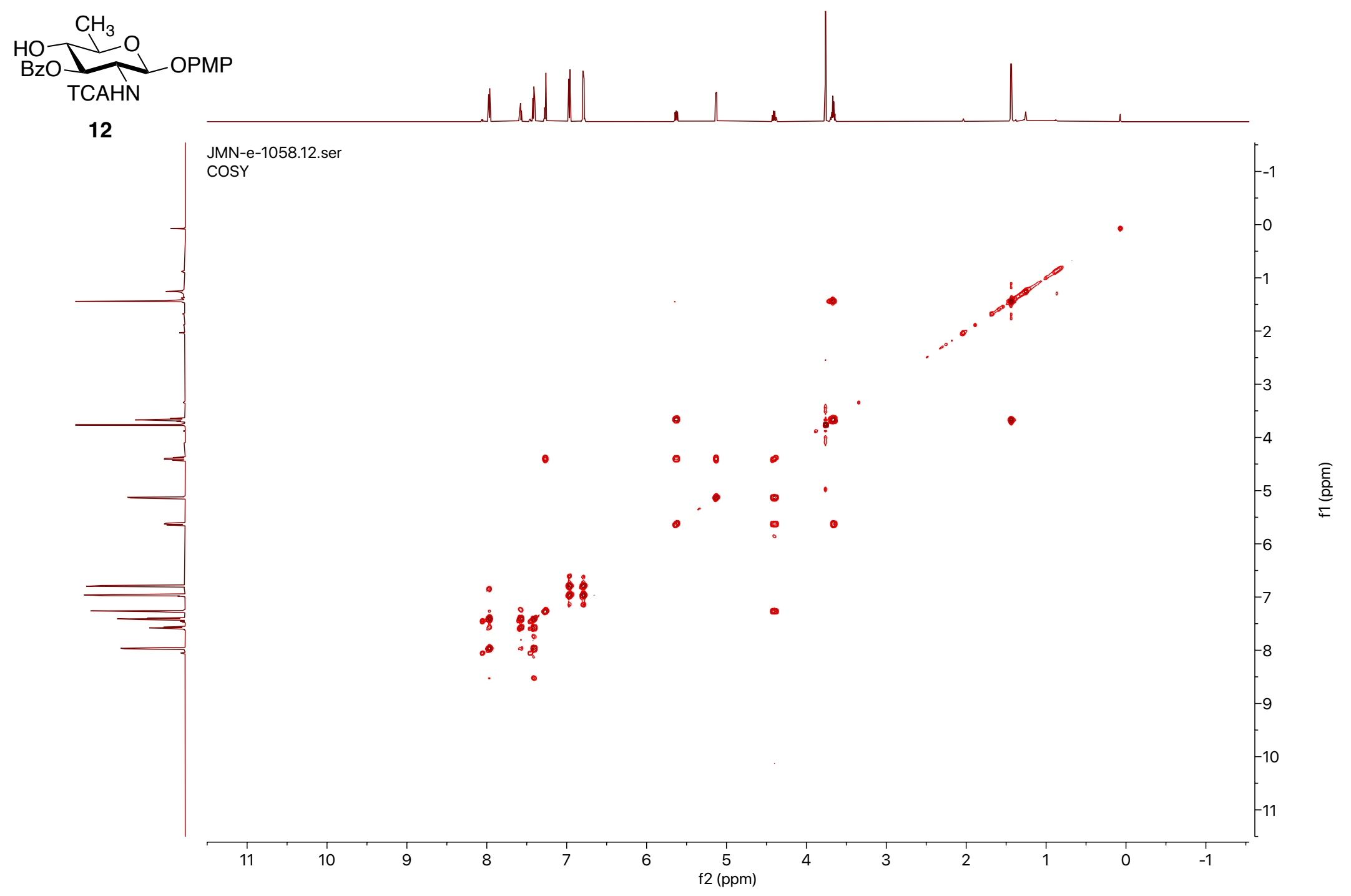


${ }^{1} \mathrm{H}-{ }^{13} \mathrm{C}$ HMBC $\left(\mathrm{CDCl}_{3}\right)$ of Compound 12

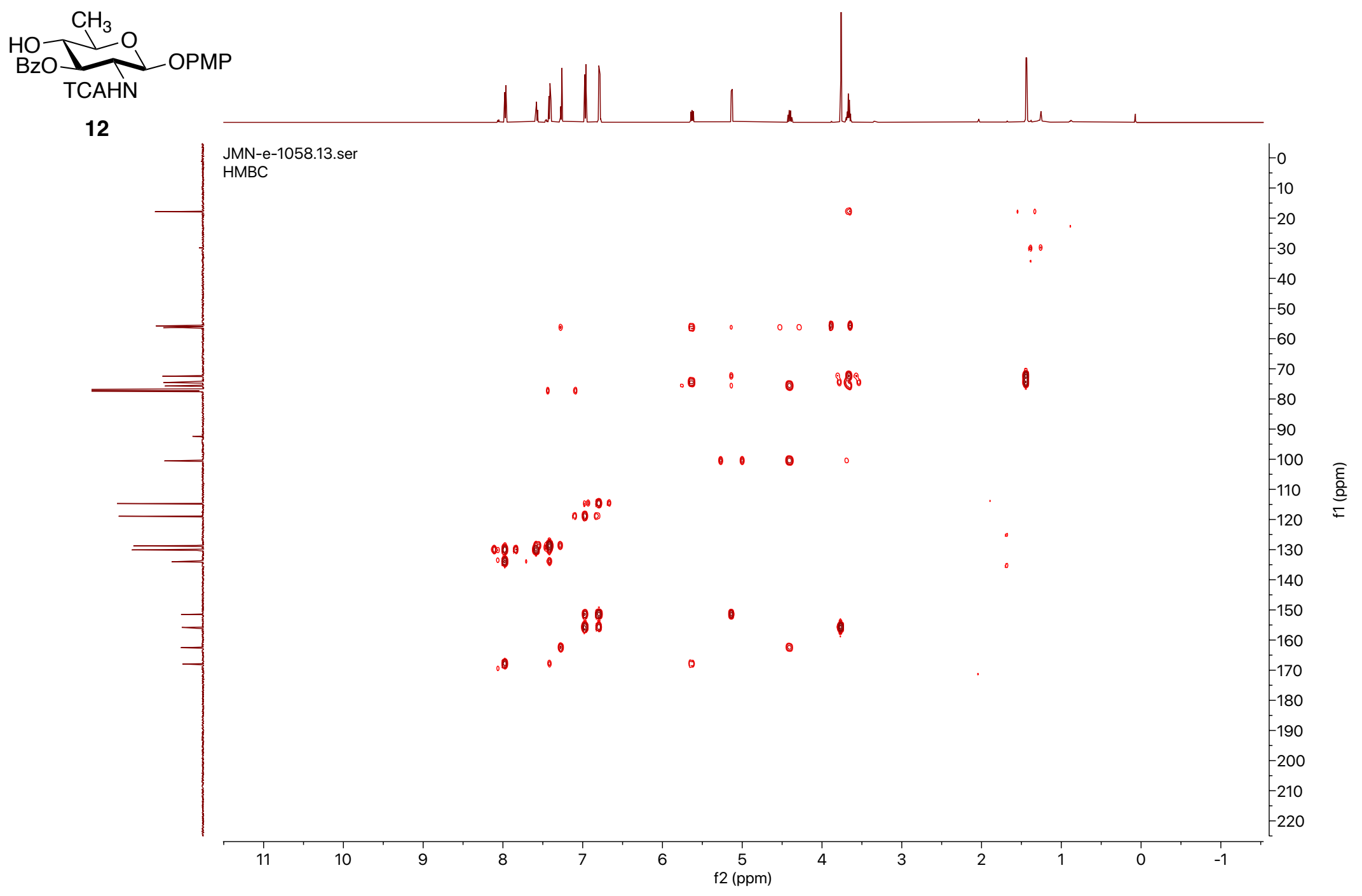


${ }^{1} \mathrm{H}$ NMR (600 MHz, $\mathrm{CDCl}_{3}$ ) of Compound 13

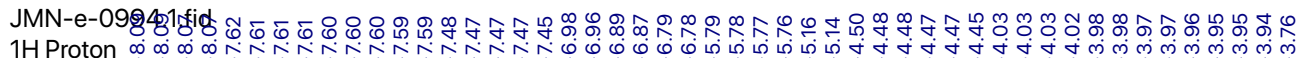

$\mathrm{N}_{3}$

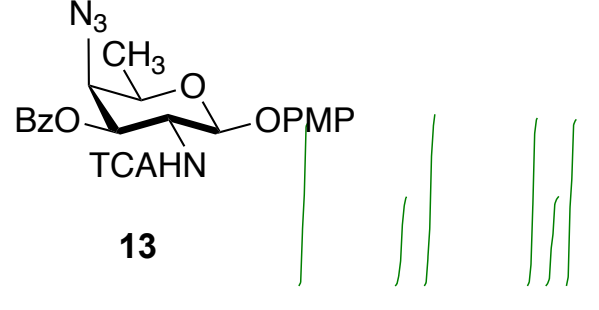

13
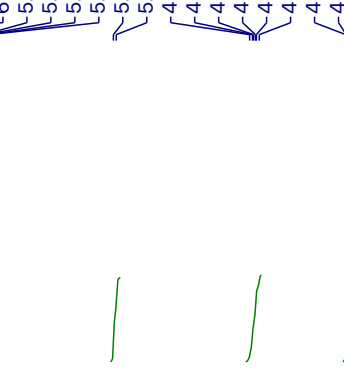

5000

$-4500$

$-4000$

$-3500$

3000

$-2500$

$-2000$

$-1500$

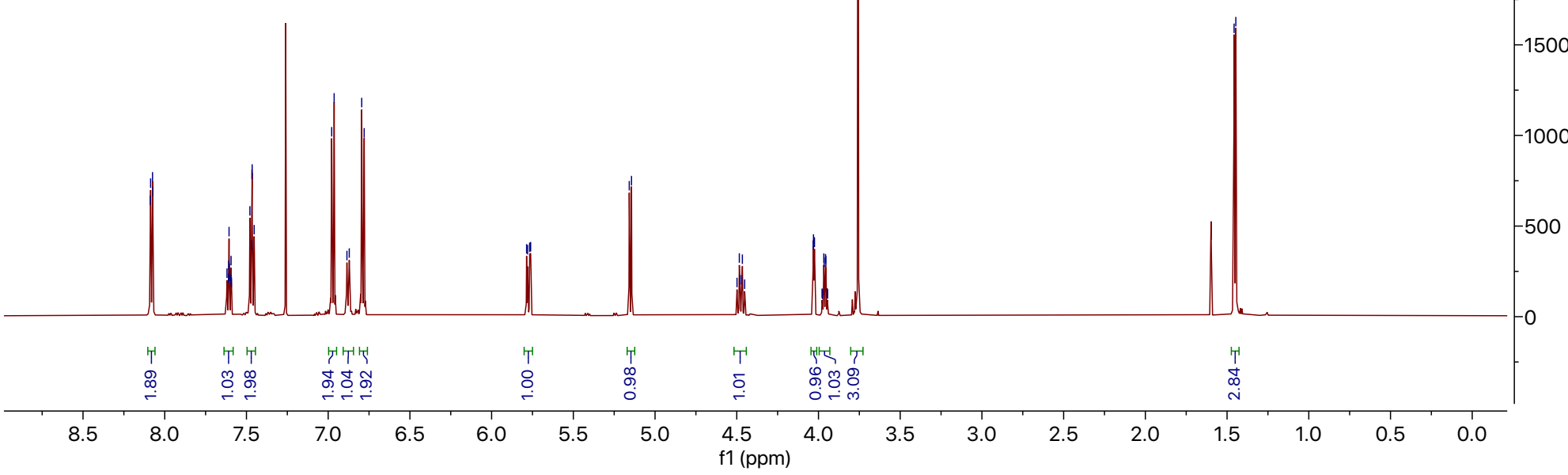


${ }^{13} \mathrm{C}$ NMR (151 MHz, $\left.\mathrm{CDCl}_{3}\right)$ of Compound 13

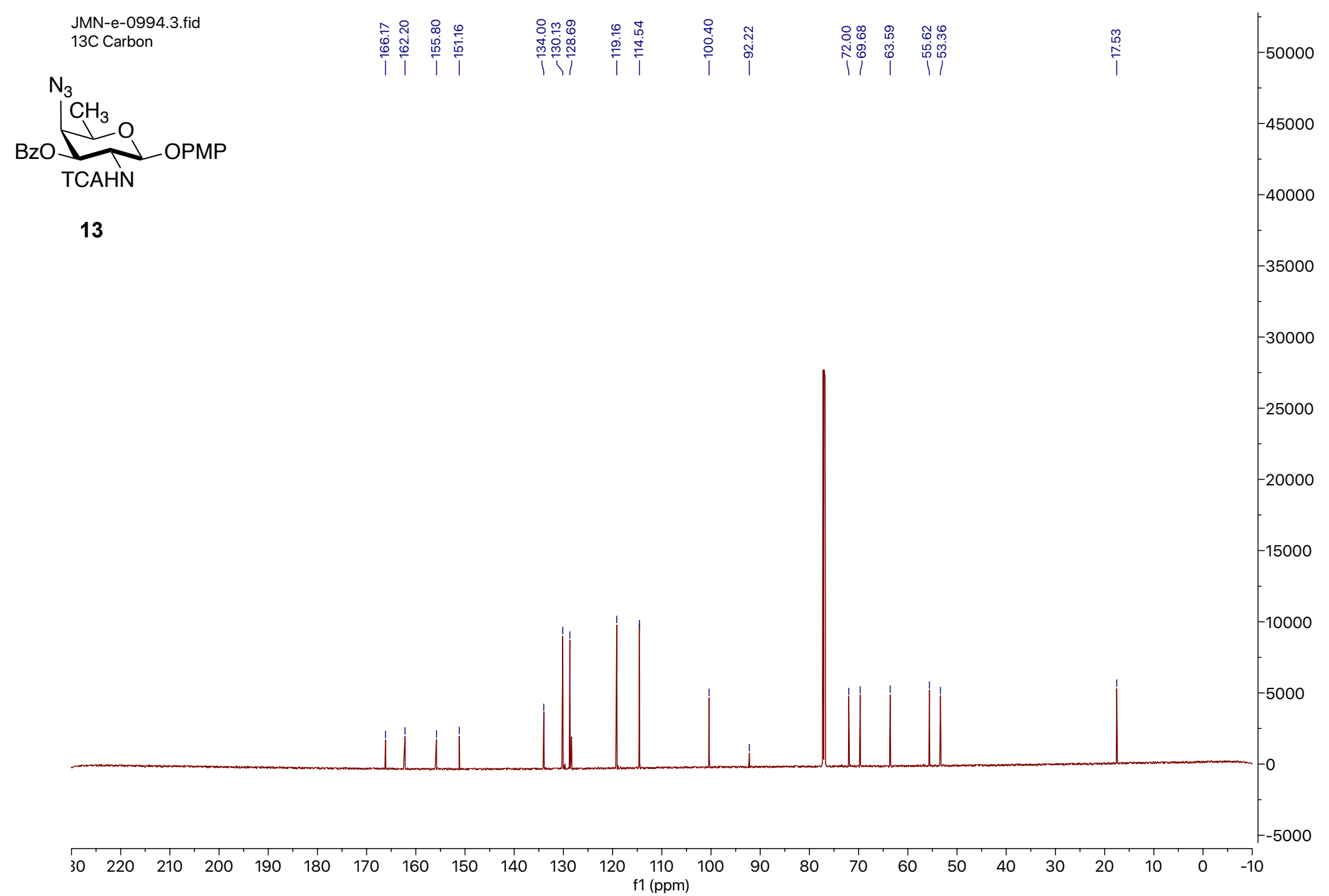


${ }^{1} \mathrm{H}-{ }^{13} \mathrm{C}$ HSQC $\left(\mathrm{CDCl}_{3}\right)$ of Compound 13

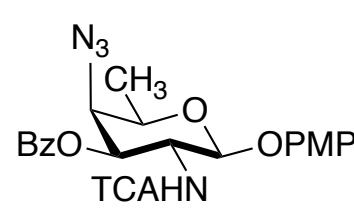

13
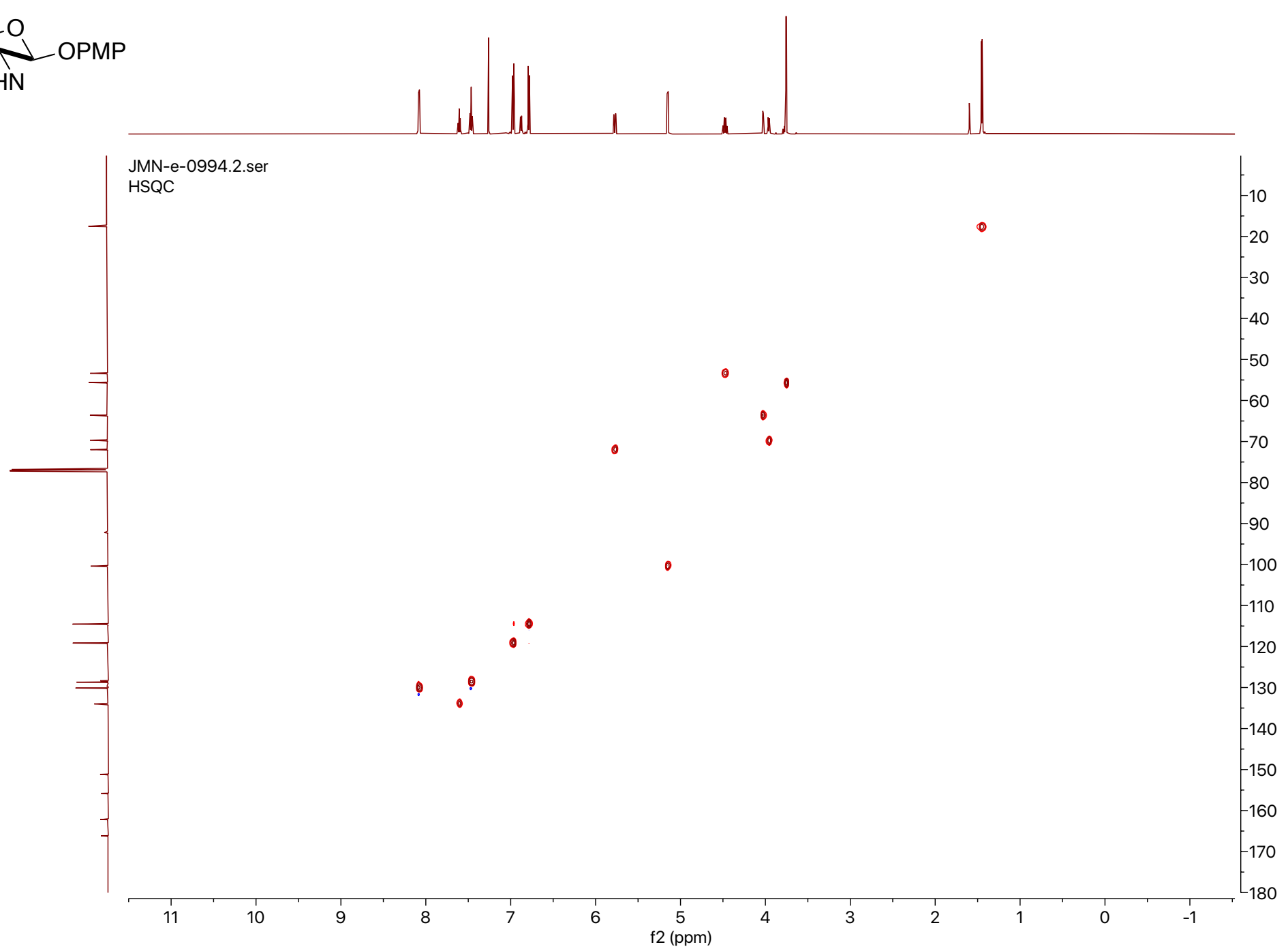

$\underset{\bar{c}}{\overline{\frac{\sigma}{0}}}$ 
${ }^{1} \mathrm{H}-{ }^{1} \mathrm{H}$ COSY $\left(\mathrm{CDCl}_{3}\right)$ of Compound 13

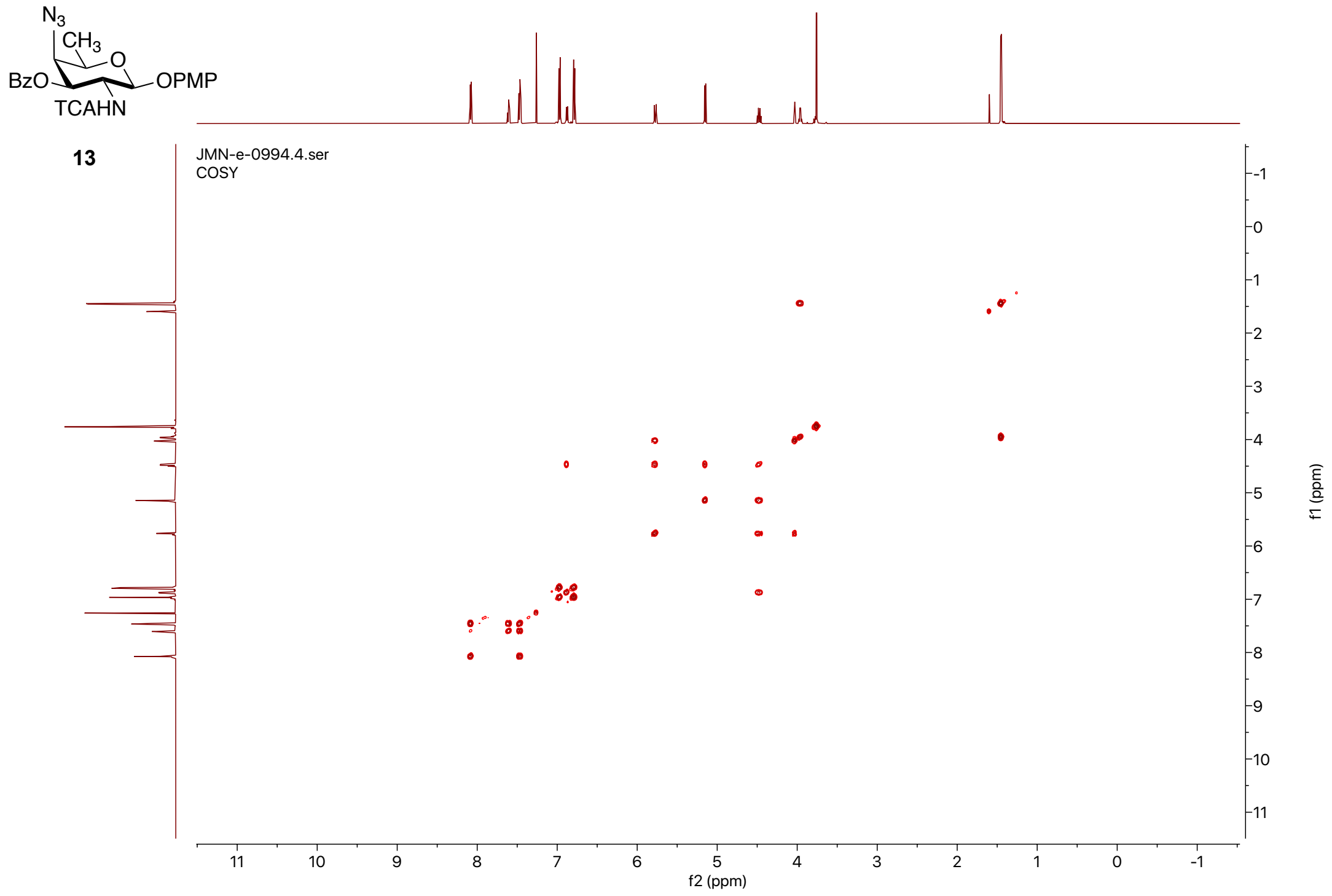


${ }^{1} \mathrm{H}-{ }^{13} \mathrm{C}$ HMBC $\left(\mathrm{CDCl}_{3}\right)$ of Compound 13

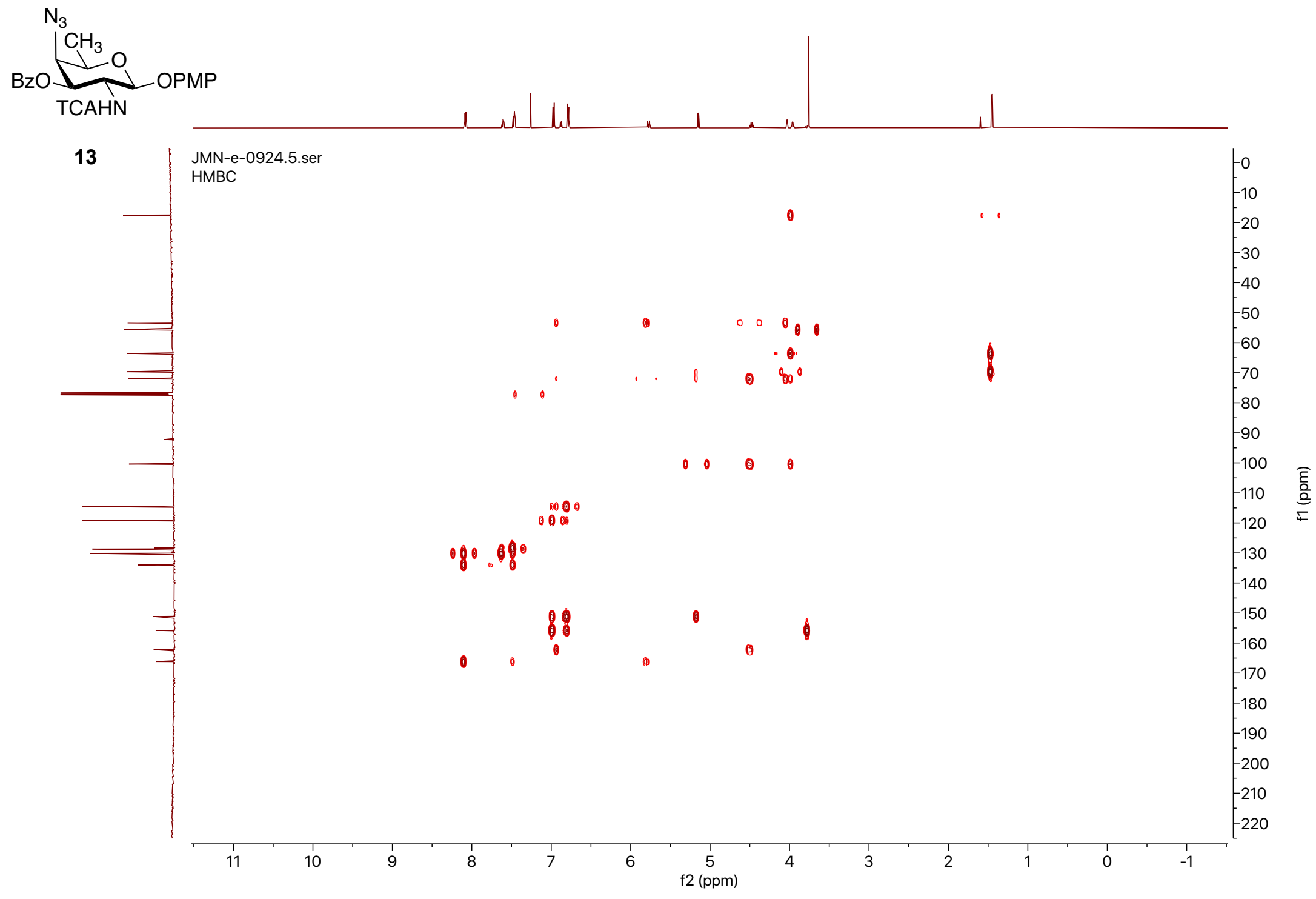


${ }^{1} \mathrm{H}$ NMR (600 MHz, $\mathrm{CDCl}_{3}$ ) of Compound 14

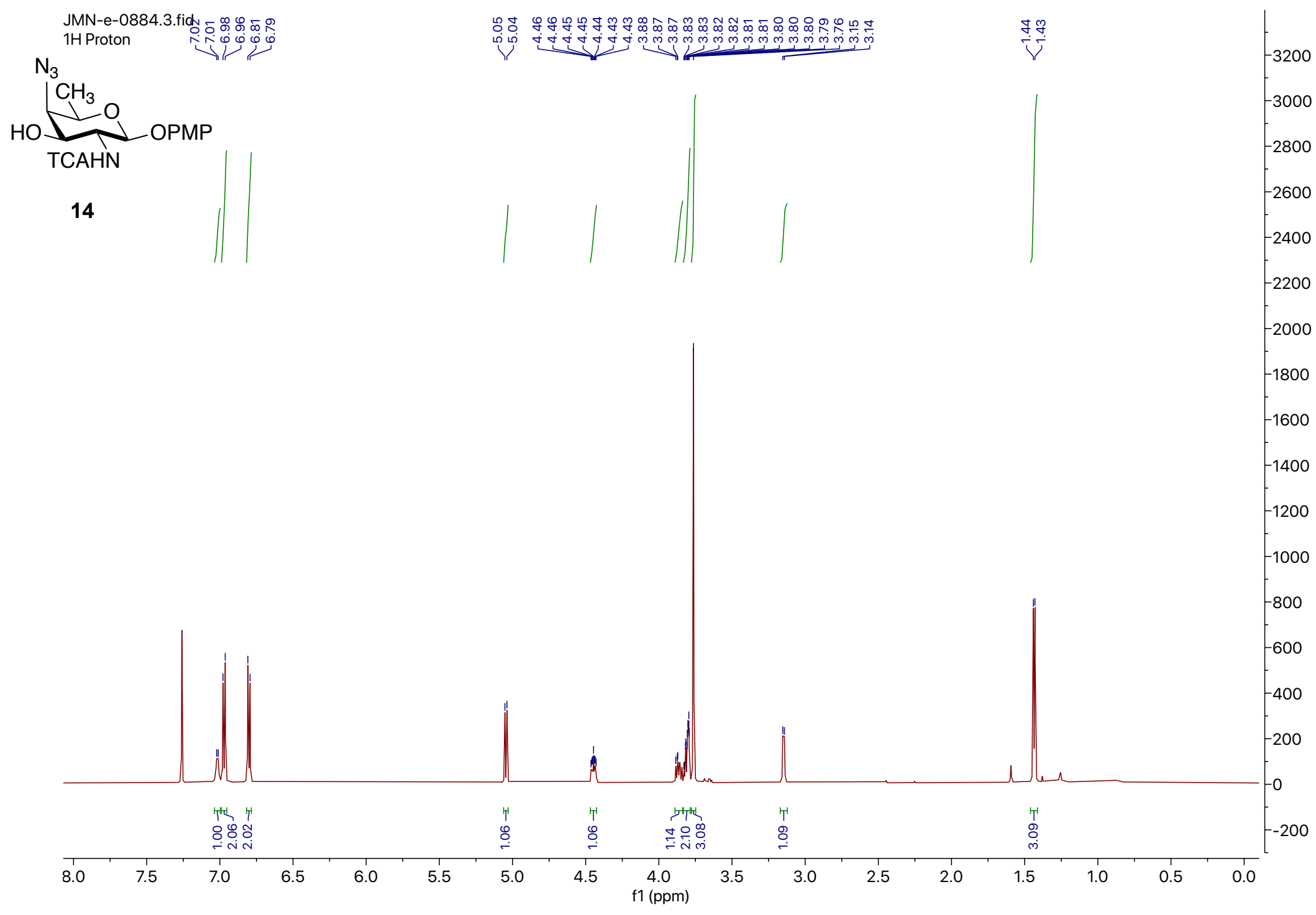


${ }^{13} \mathrm{C}$ NMR (151 MHz, $\mathrm{CDCl}_{3}$ ) of Compound 14

JMN-e-0884.4.fid

$13 \mathrm{C}$ Carbon

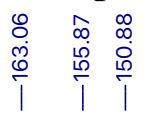



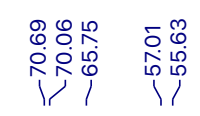

TCAHN

14

\section{4}

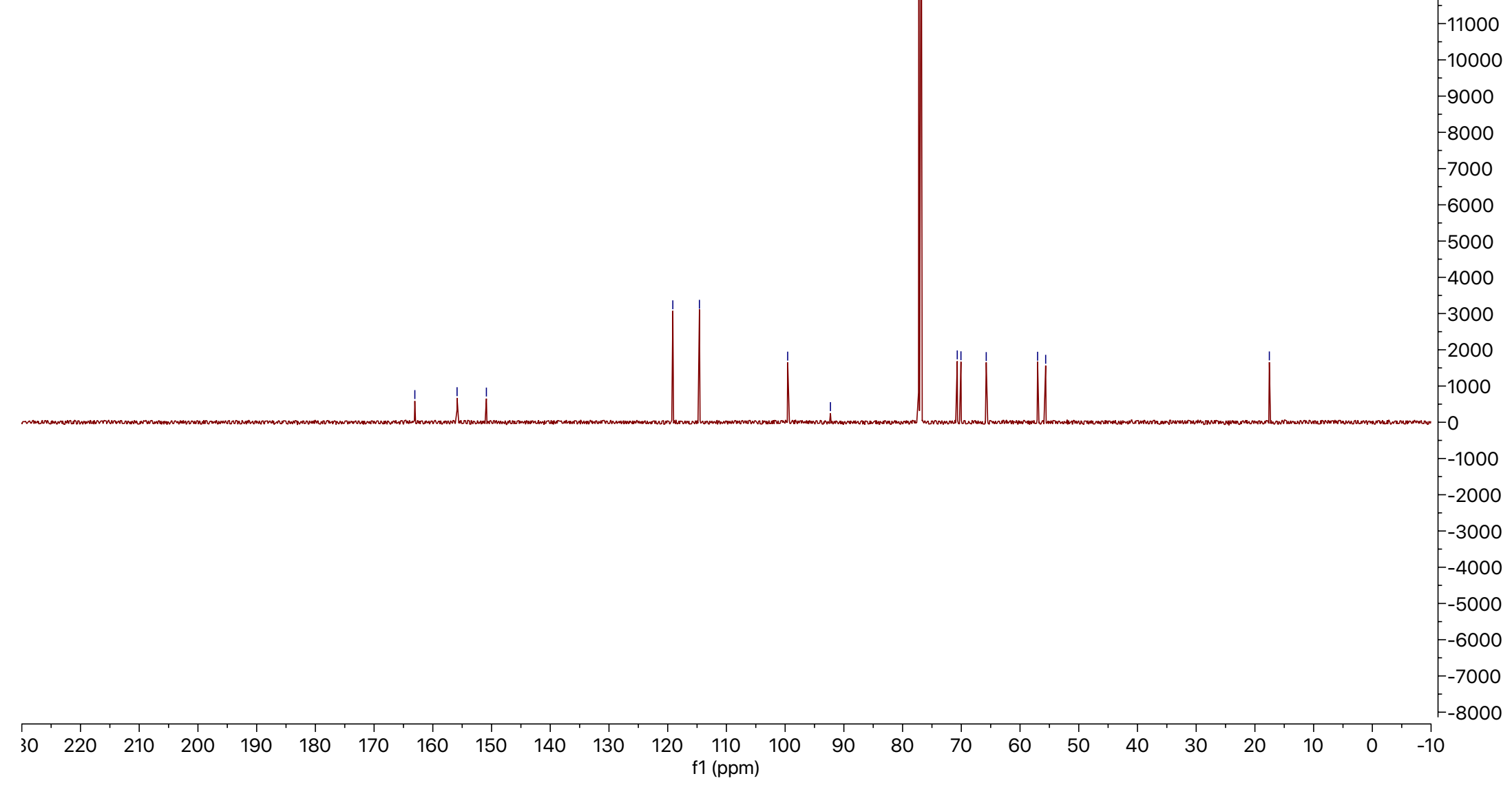


${ }^{1} \mathrm{H}-{ }^{13} \mathrm{C}$ HSQC $\left(\mathrm{CDCl}_{3}\right)$ of Compound 14

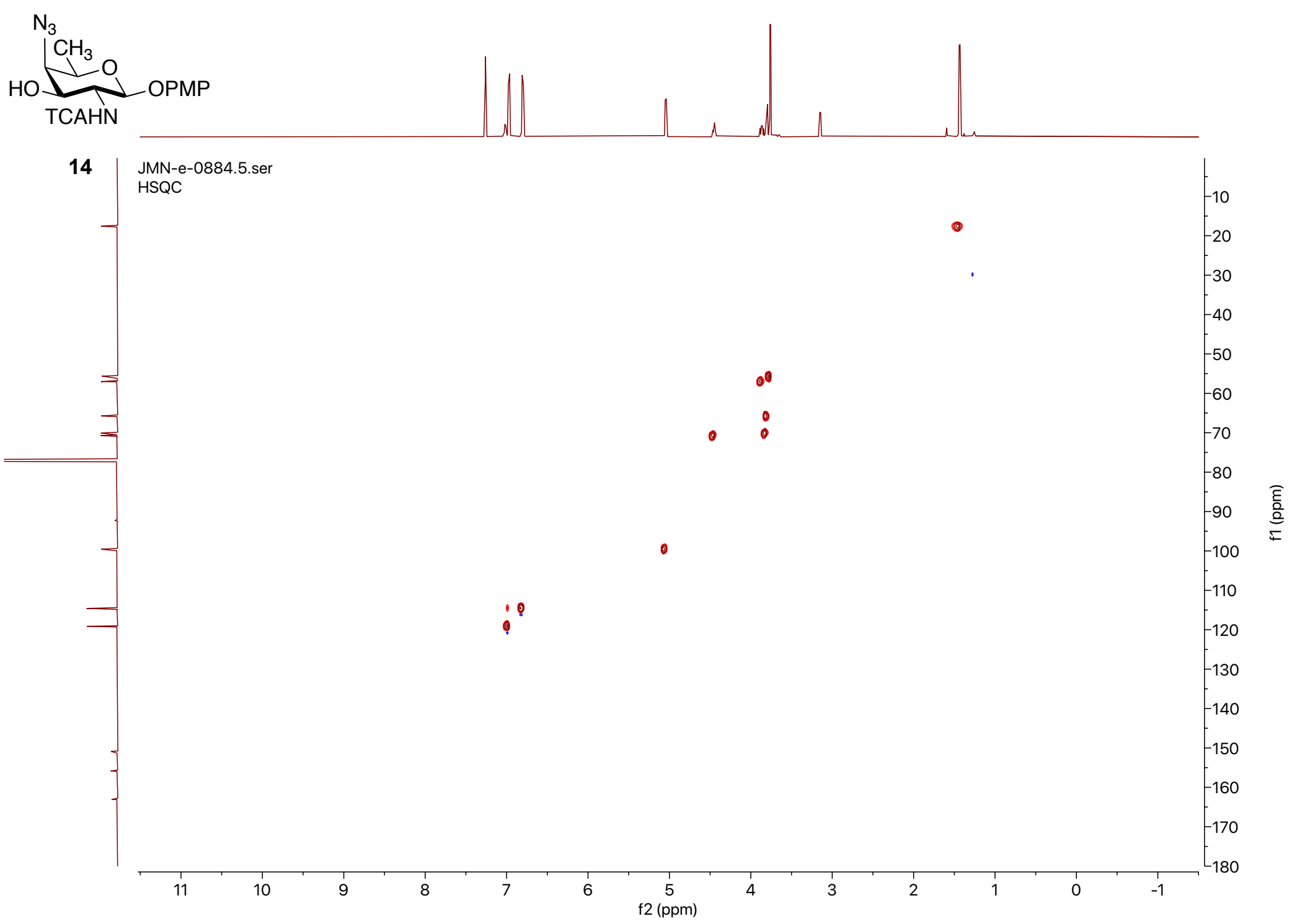


${ }^{1} \mathrm{H}-{ }^{1} \mathrm{H}$ COSY $\left(\mathrm{CDCl}_{3}\right)$ of Compound 14

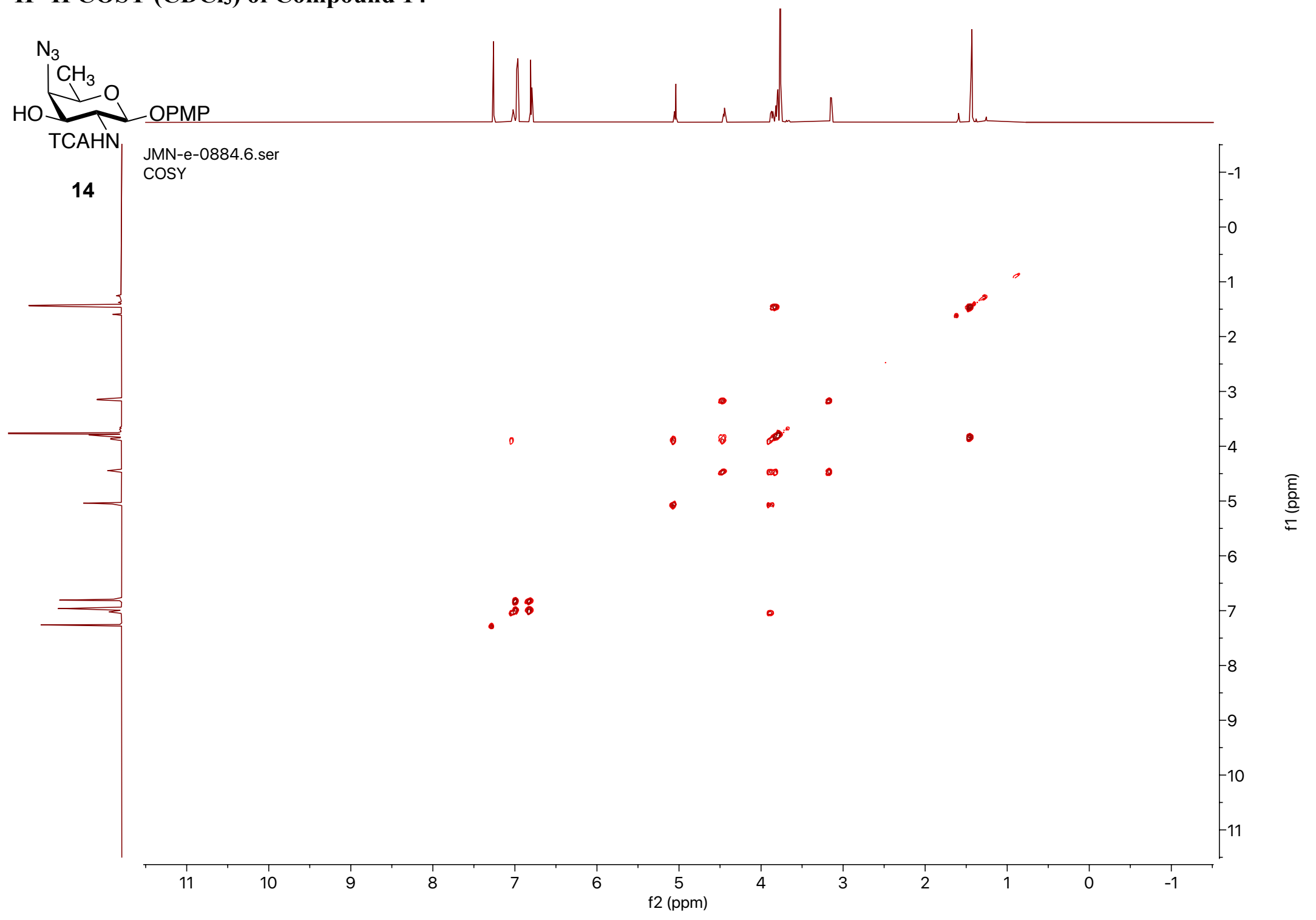


${ }^{1} \mathrm{H}$ NMR (600 MHz, $\left.\mathrm{CDCl}_{3}\right)$ of Compound 15

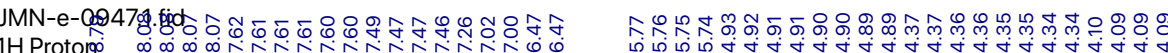

1H Protolo $\underbrace{\infty}$

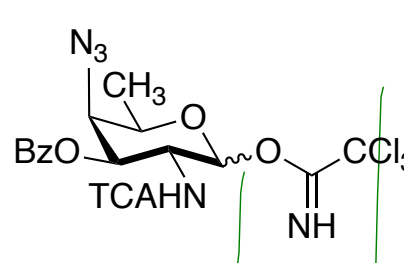

15
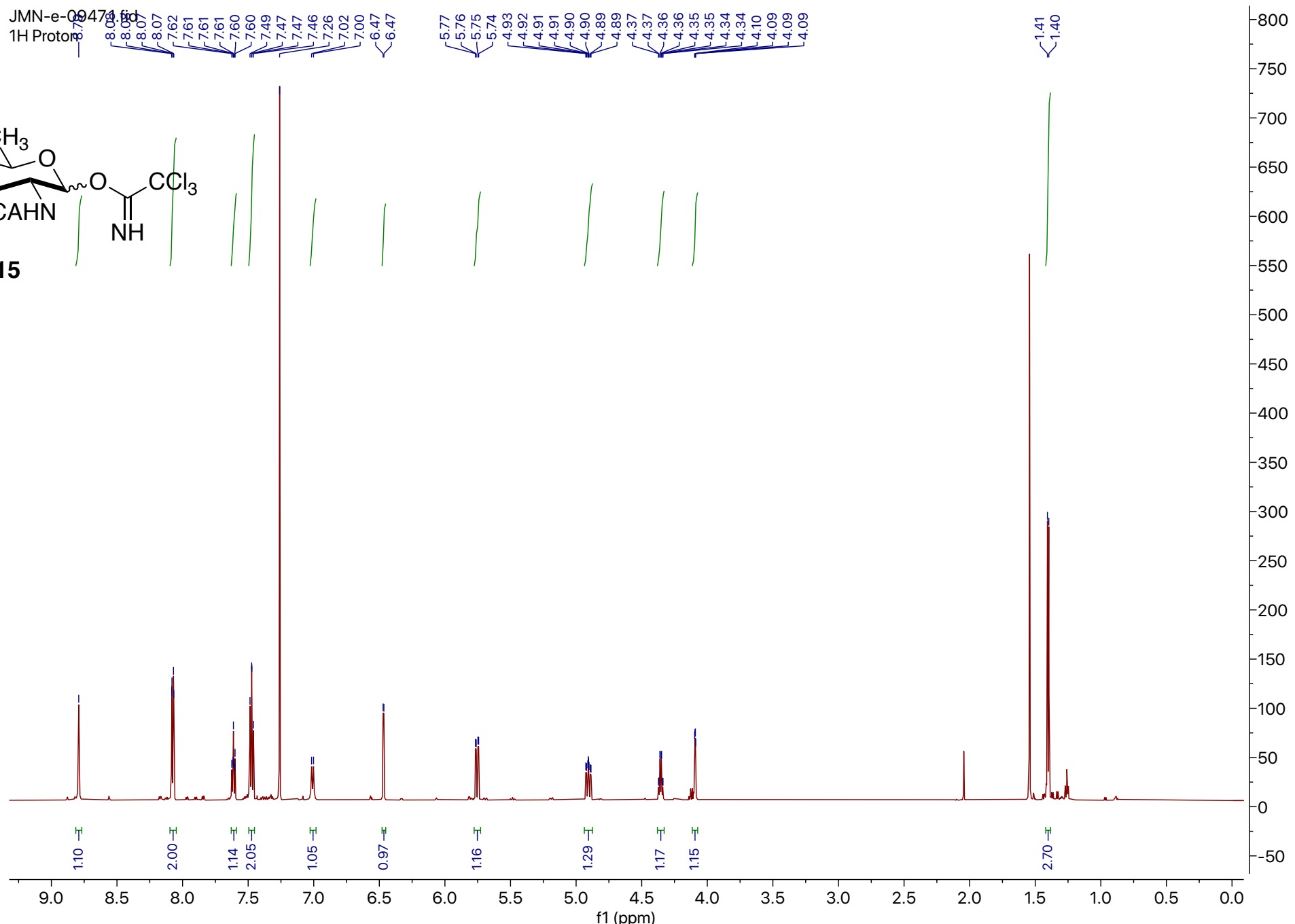
${ }^{13} \mathrm{C}$ NMR (151 MHz, $\left.\mathrm{CDCl}_{3}\right)$ of Compound 15

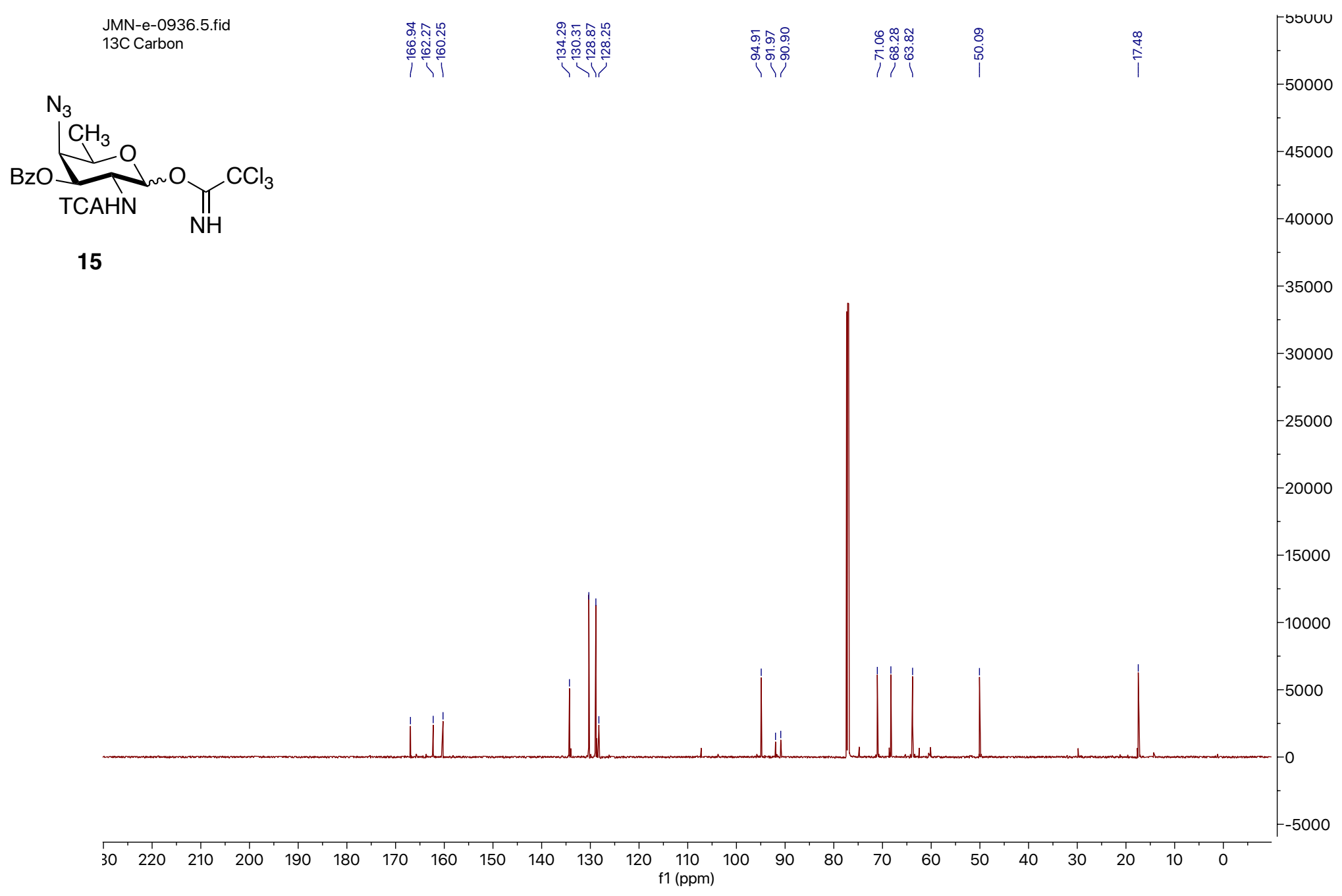


${ }^{1} \mathrm{H}-{ }^{13} \mathrm{C}$ HSQC $\left(\mathrm{CDCl}_{3}\right)$ of Compound 15

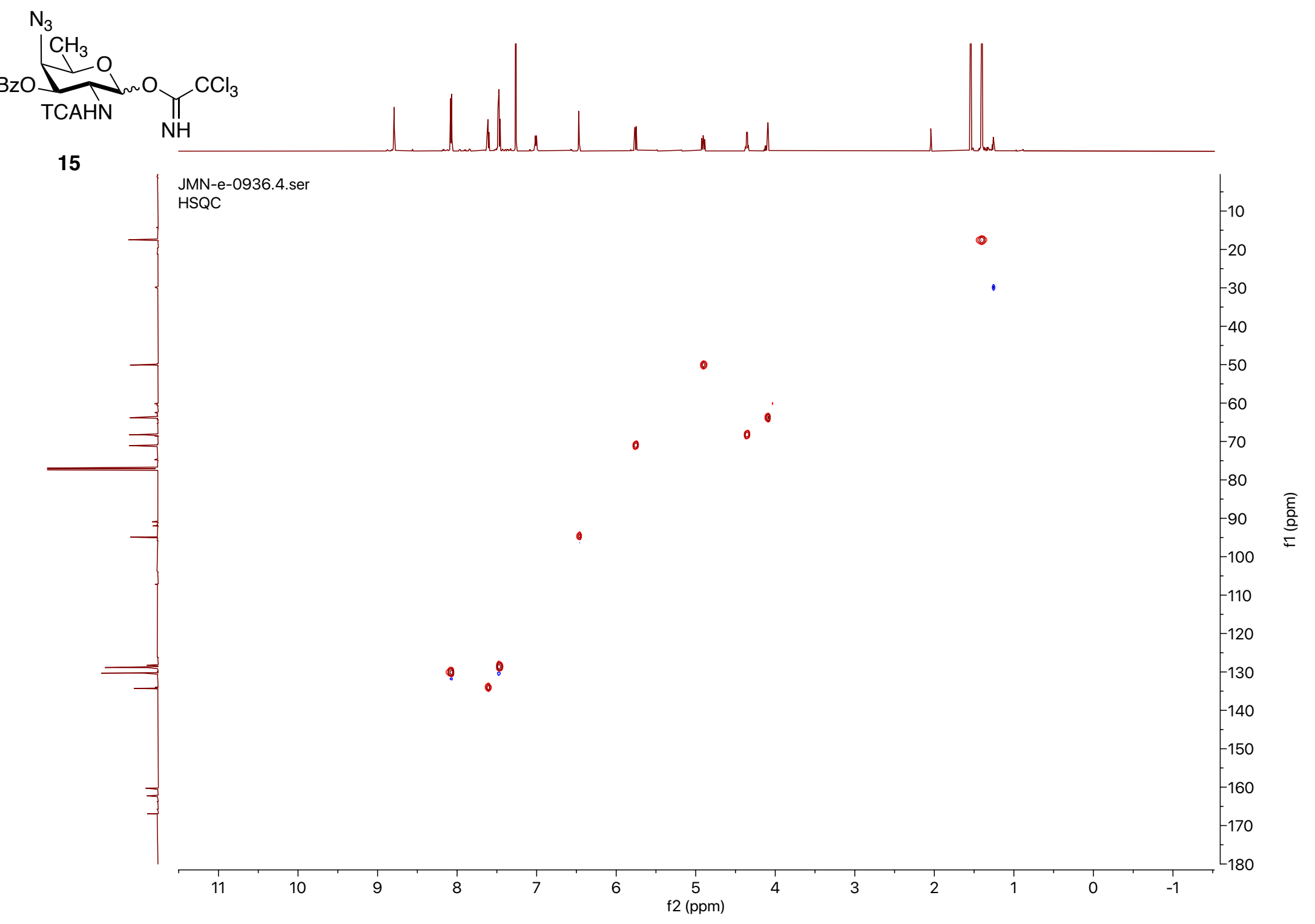


${ }^{1} \mathrm{H}$ NMR (600 MHz, $\left.\mathrm{CDCl}_{3}\right)$ of Compound 17

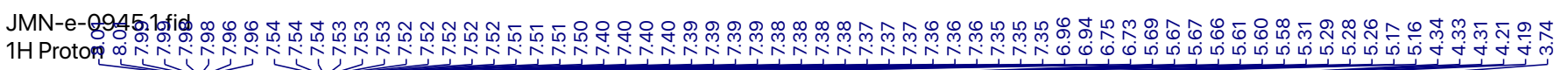
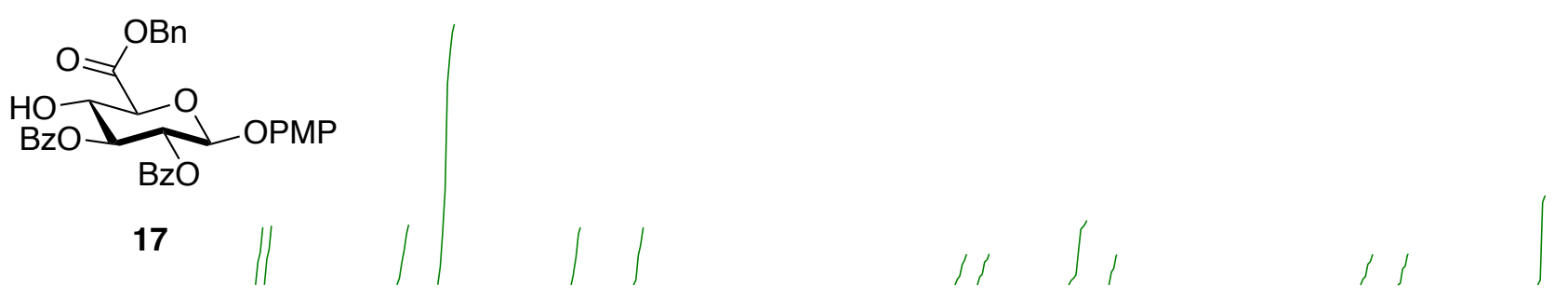

17

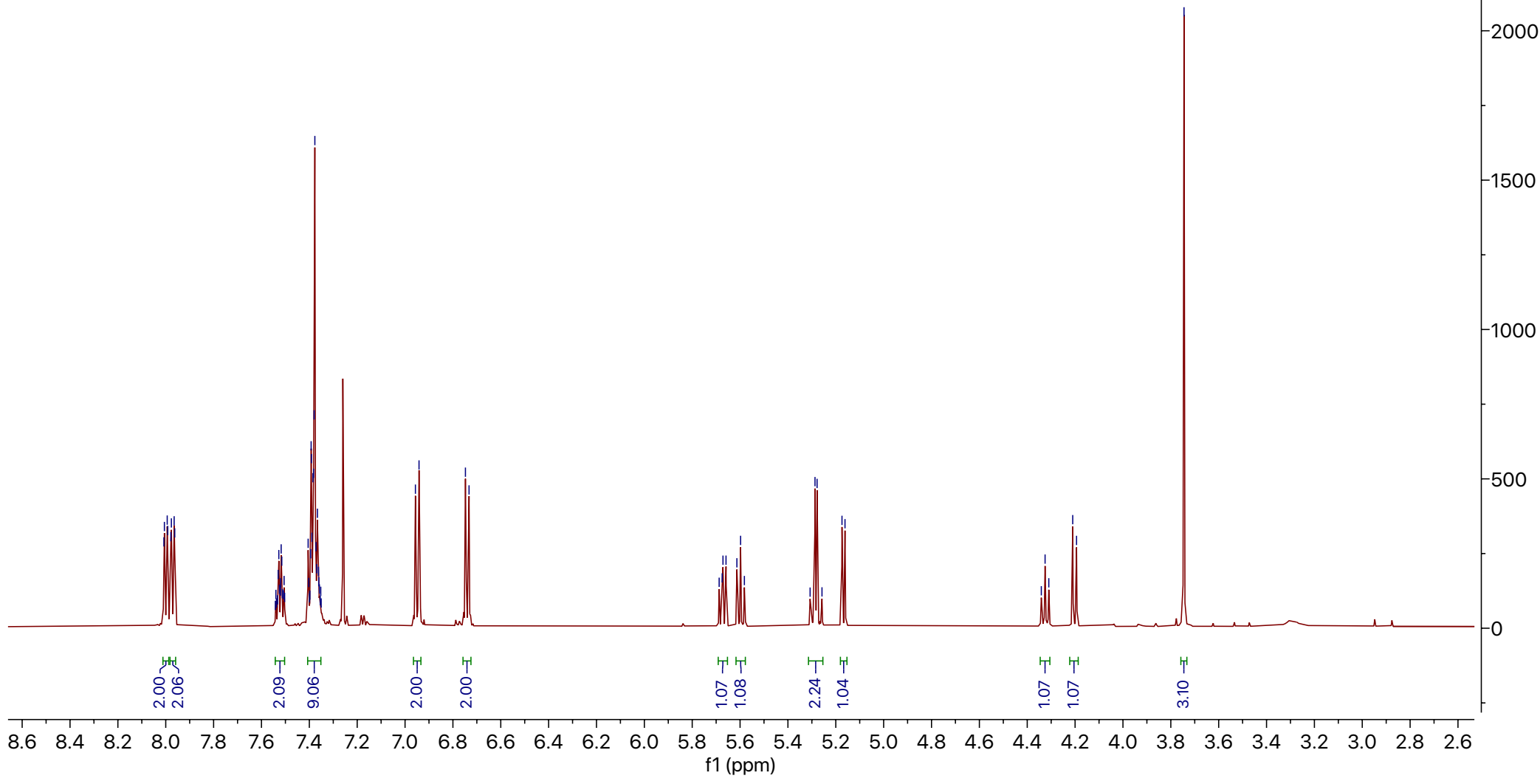


${ }^{13} \mathrm{C}$ NMR (151 MHz, $\left.\mathrm{CDCl}_{3}\right)$ of Compound 17

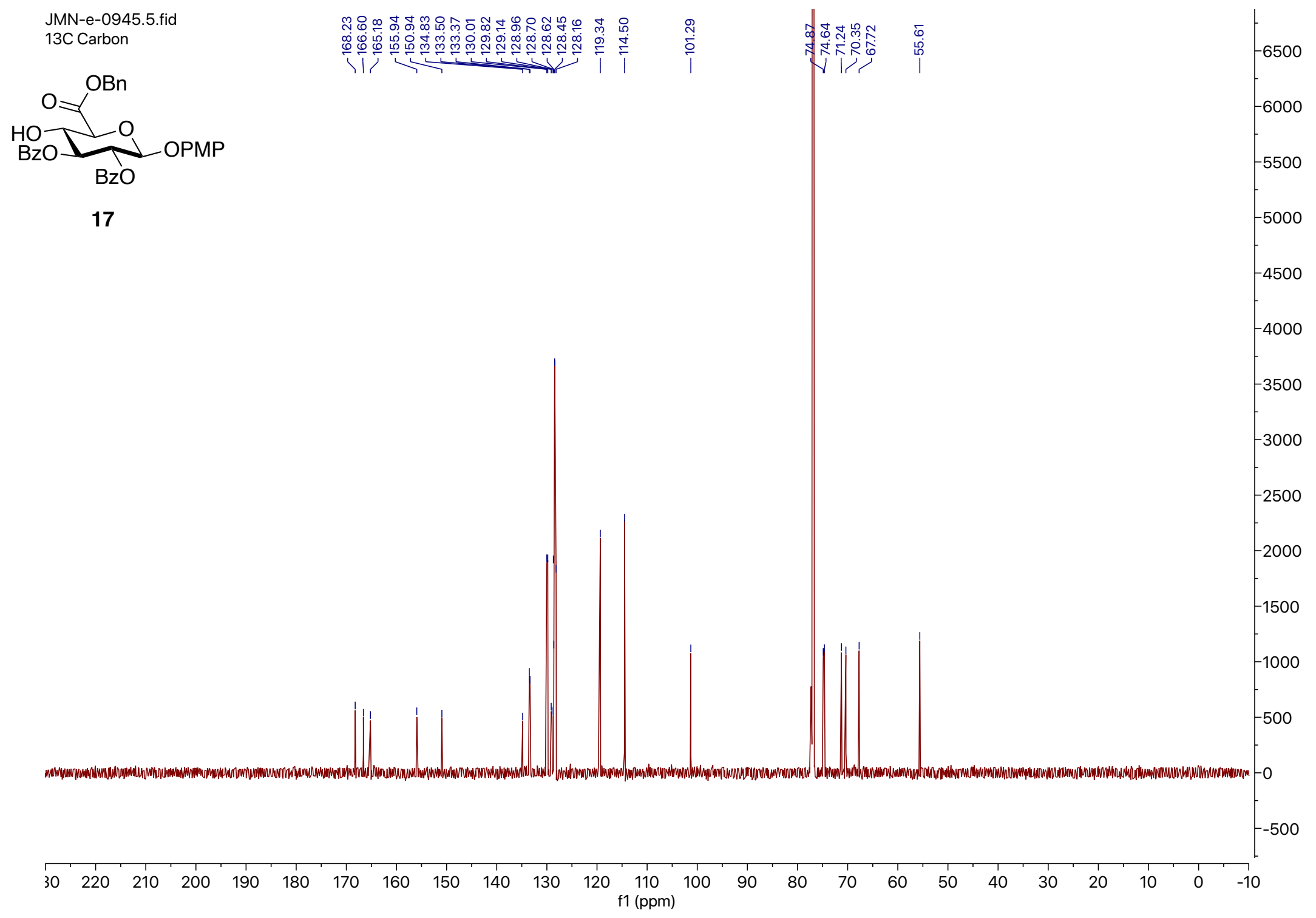


${ }^{1} \mathrm{H}-{ }^{13} \mathrm{C}$ HSQC $\left(\mathrm{CDCl}_{3}\right)$ of $\mathrm{Compound} 17$

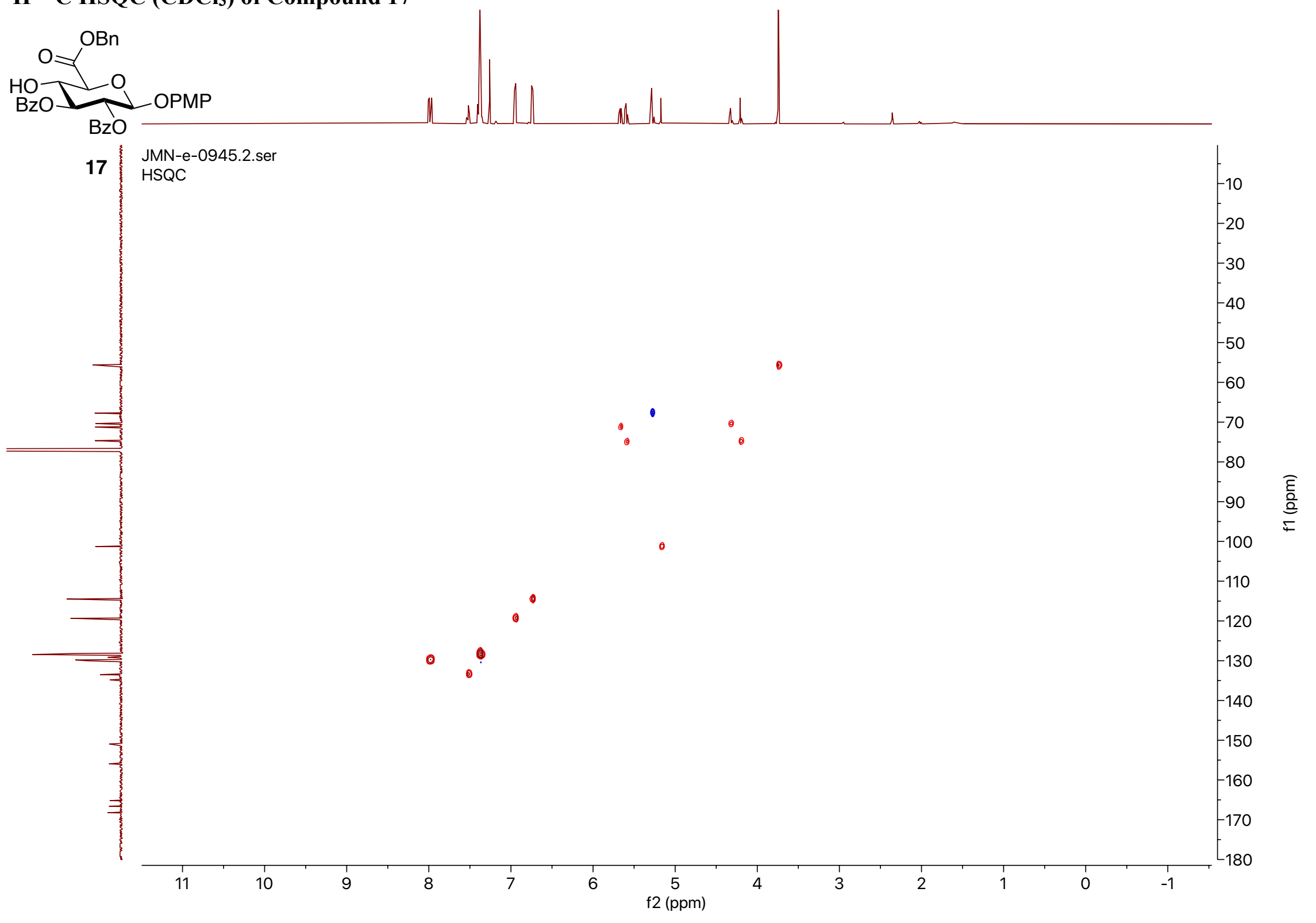


${ }^{1} \mathrm{H}$ NMR (600 MHz, $\mathrm{CDCl}_{3}$ ) of Compound 18

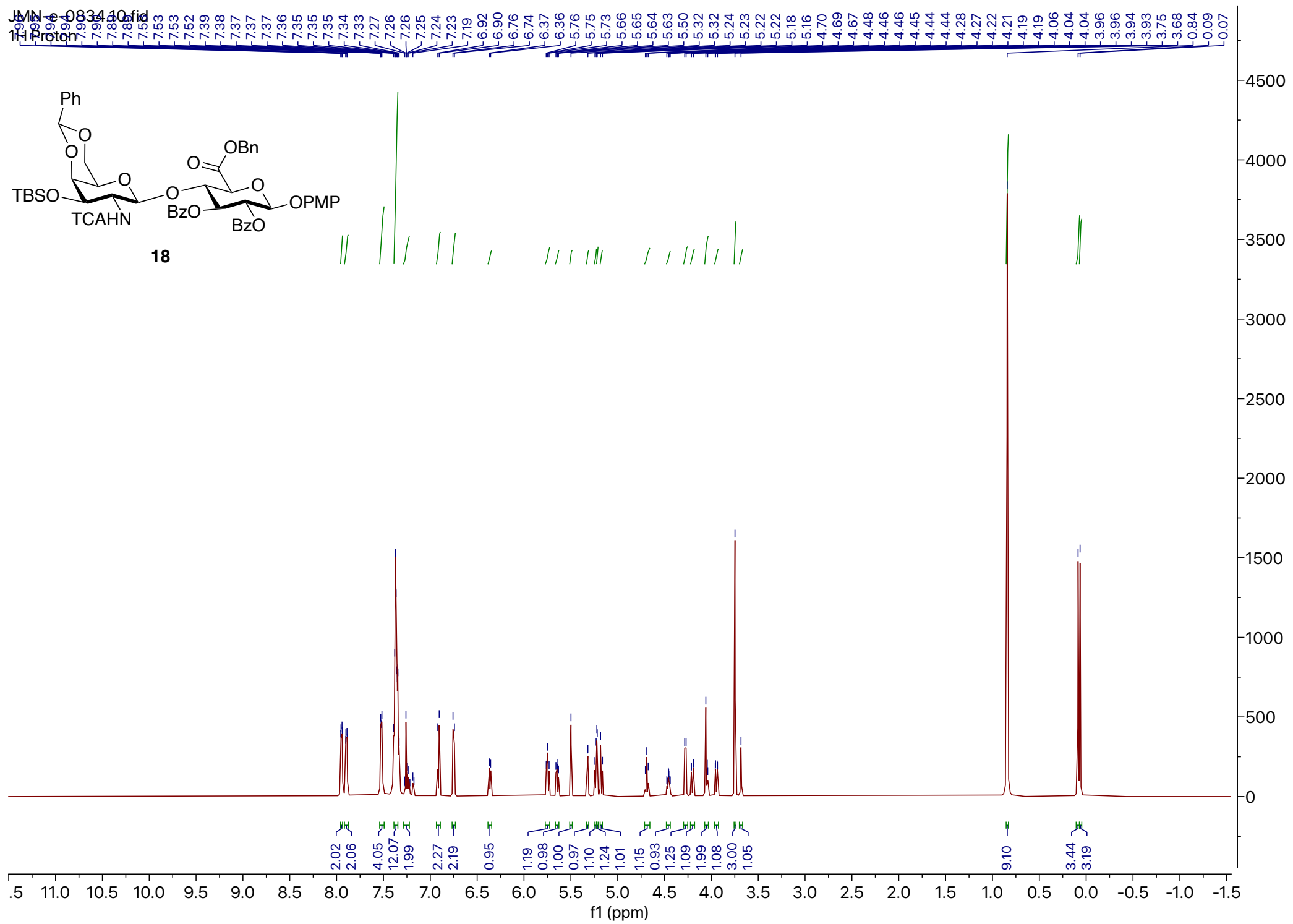


${ }^{13} \mathrm{C}$ NMR (151 MHz, $\left.\mathrm{CDCl}_{3}\right)$ of Compound 18

JMN-e-0834.11.fid $13 \mathrm{C}$ Carbon

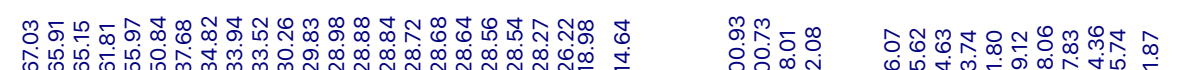

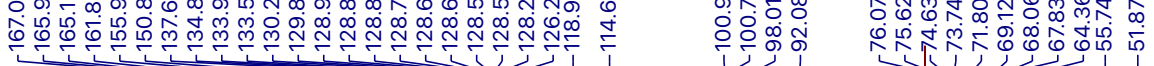

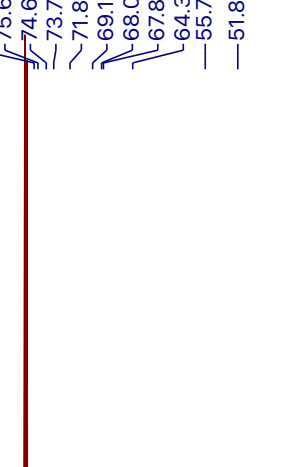

18
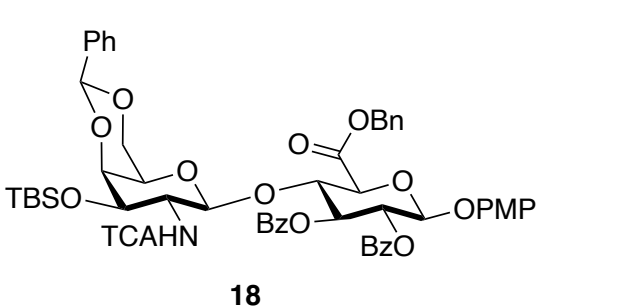
${ }^{1} \mathrm{H}-{ }^{13} \mathrm{C}$ HSQC $\left(\mathrm{CDCl}_{3}\right)$ of $\mathrm{Compound} 18$

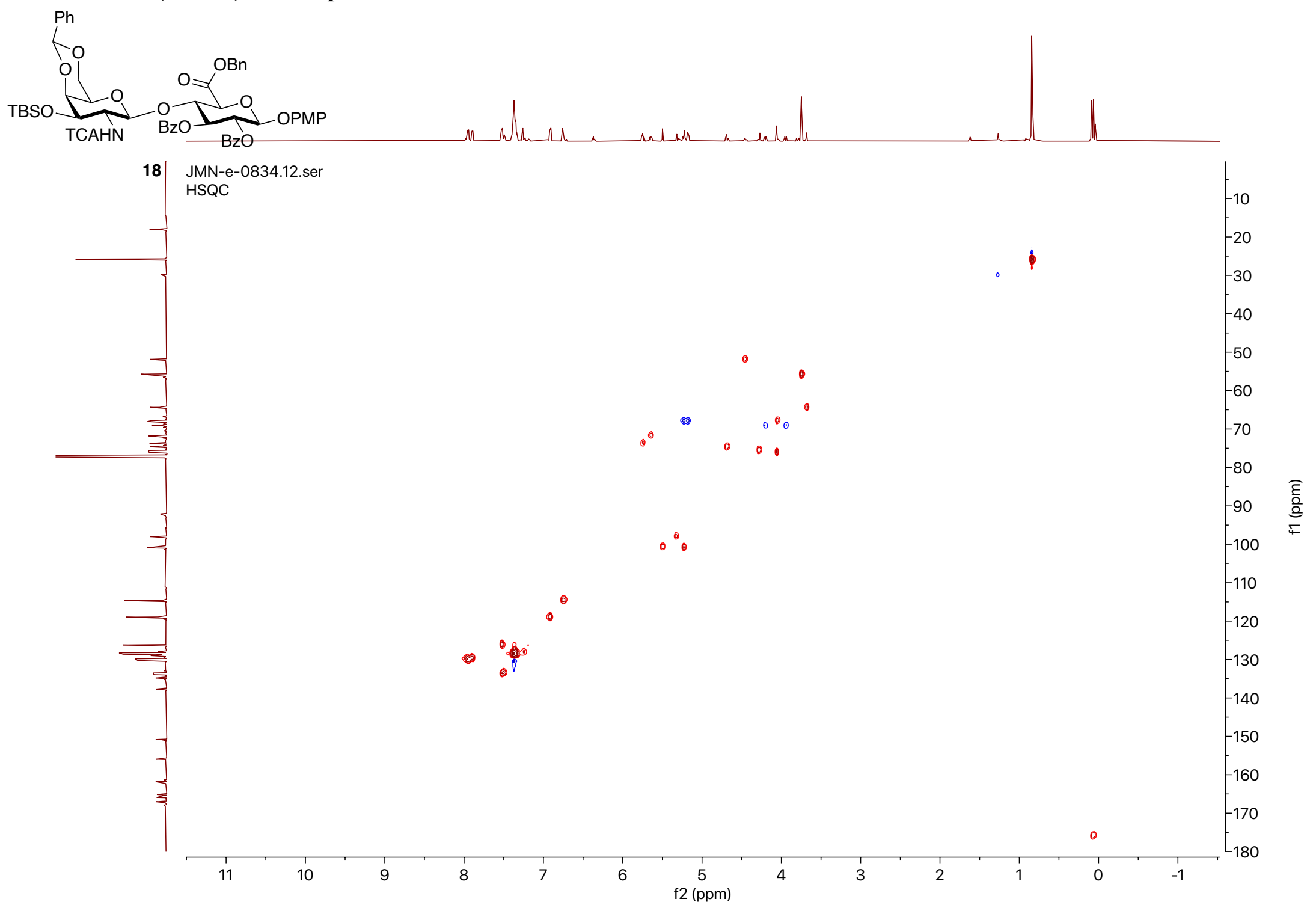


${ }^{1} \mathrm{H}$ NMR (600 MHz, $\mathrm{CDCl}_{3}$ ) of Compound 19

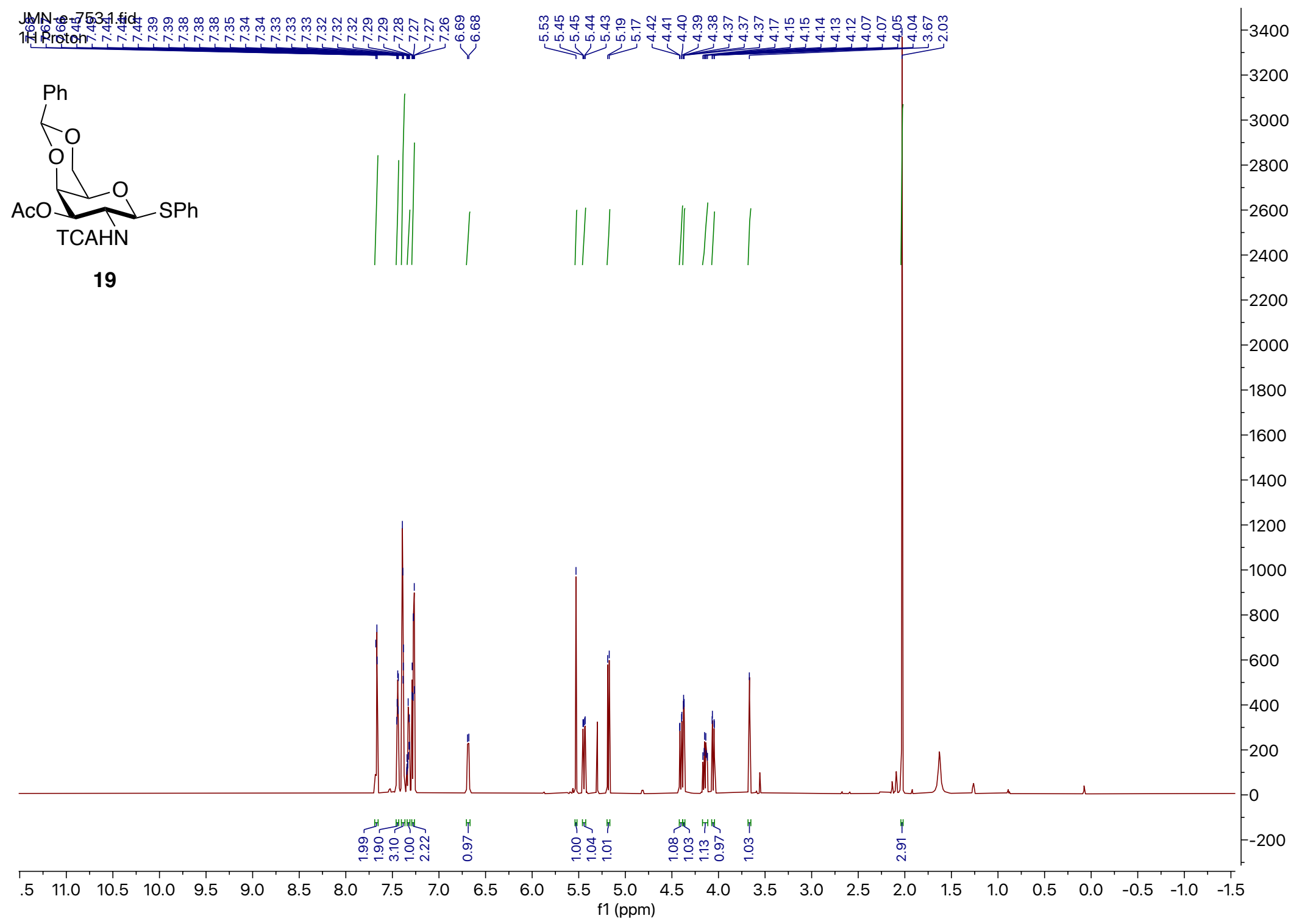


${ }^{13} \mathrm{C}$ NMR (151 MHz, $\left.\mathrm{CDCl}_{3}\right)$ of Compound 19

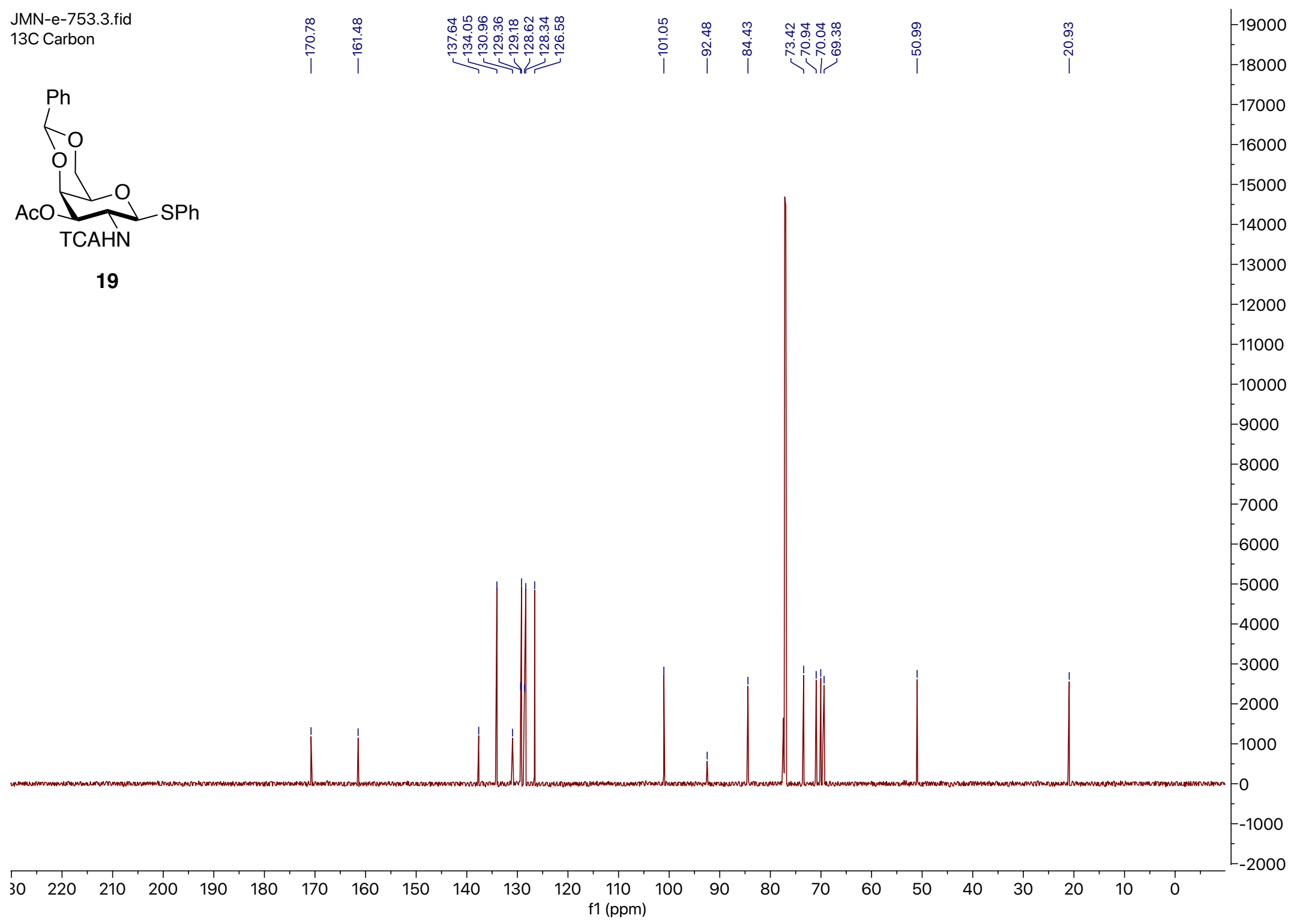


${ }^{1} \mathrm{H}-{ }^{13} \mathrm{C}$ HSQC $\left(\mathrm{CDCl}_{3}\right)$ of Compound 19

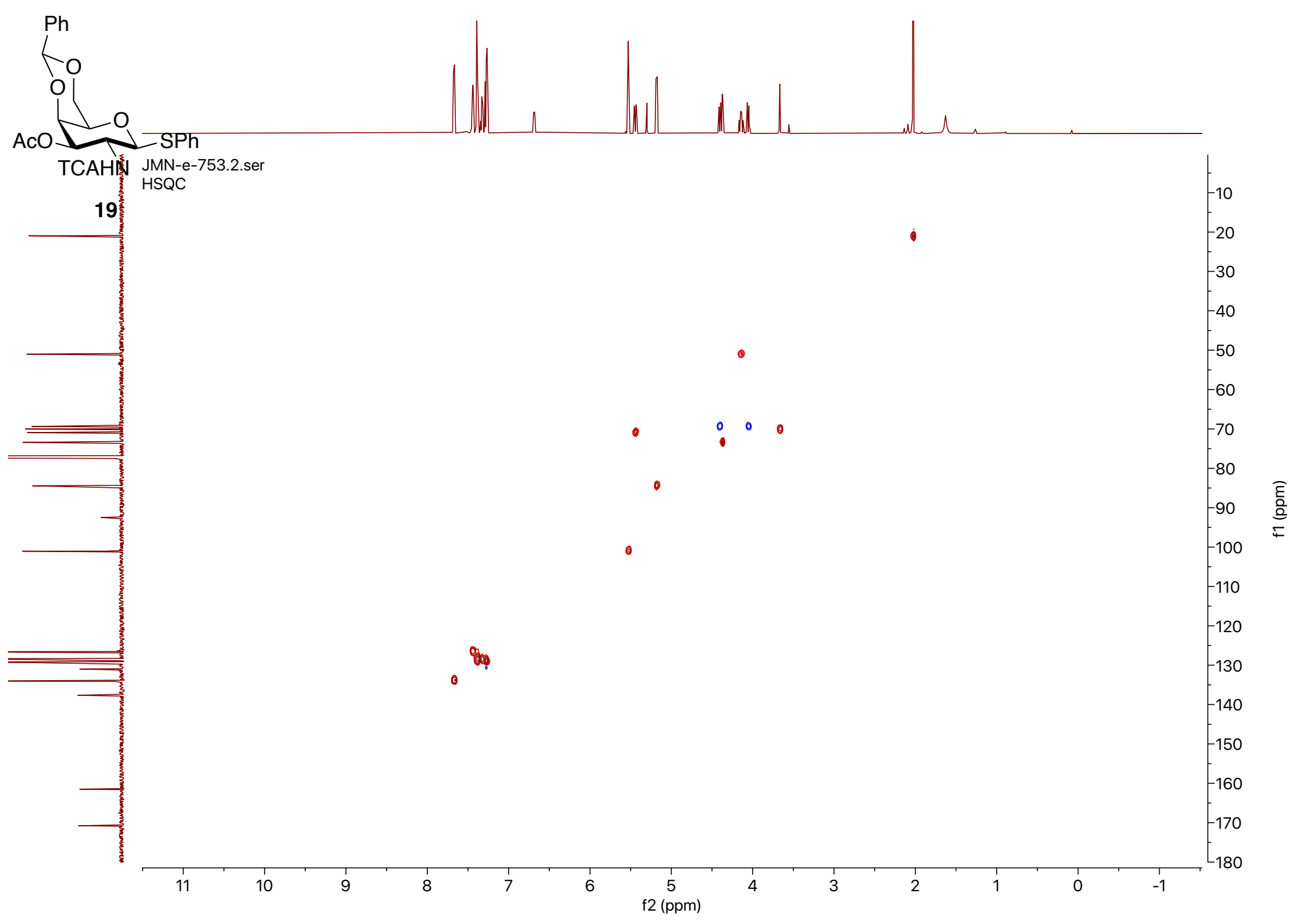


${ }^{1} \mathrm{H}$ NMR (600 MHz, $\mathrm{CDCl}_{3}$ ) of Compound 20

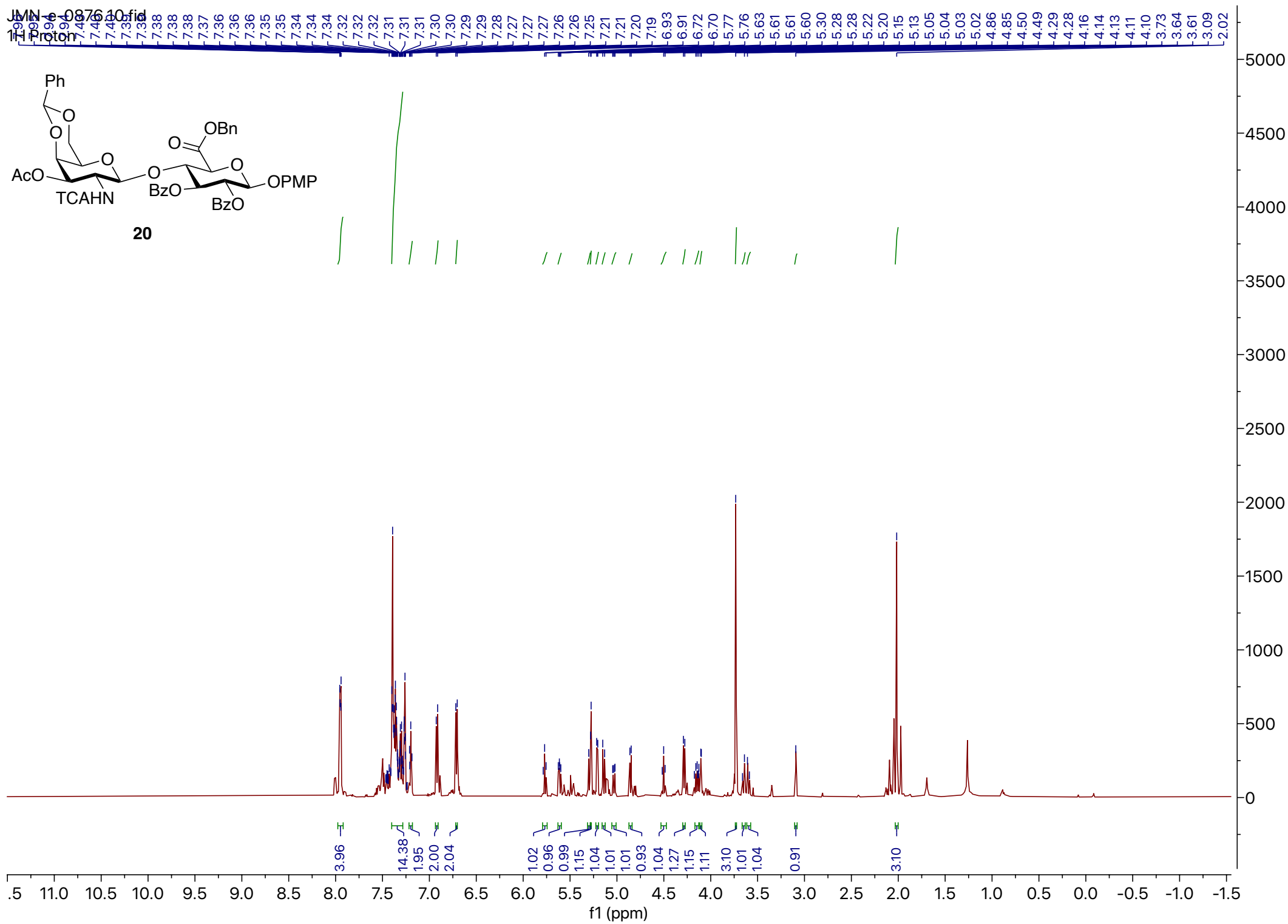


${ }^{13} \mathrm{C}$ NMR (151 MHz, $\left.\mathrm{CDCl}_{3}\right)$ of Compound 20 $13 \mathrm{C}$ Carbon

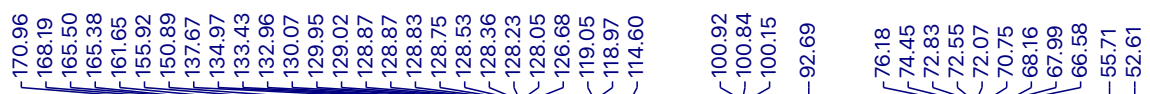

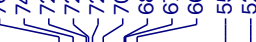

23000

22000

21000

20000

TCAHN

20

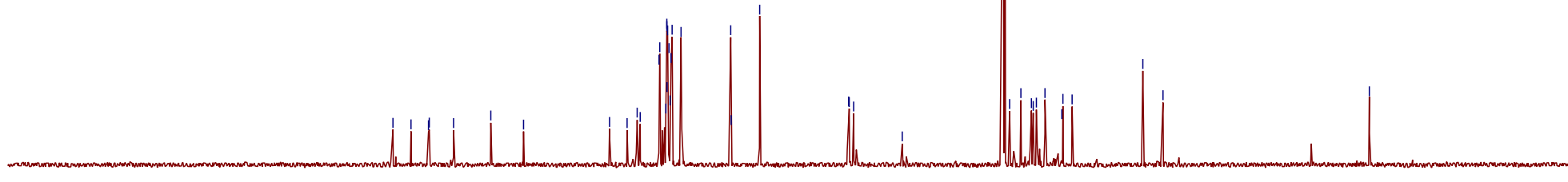


${ }^{1} \mathrm{H}-{ }^{13} \mathrm{C}$ HSQC $\left(\mathrm{CDCl}_{3}\right)$ of Compound 20

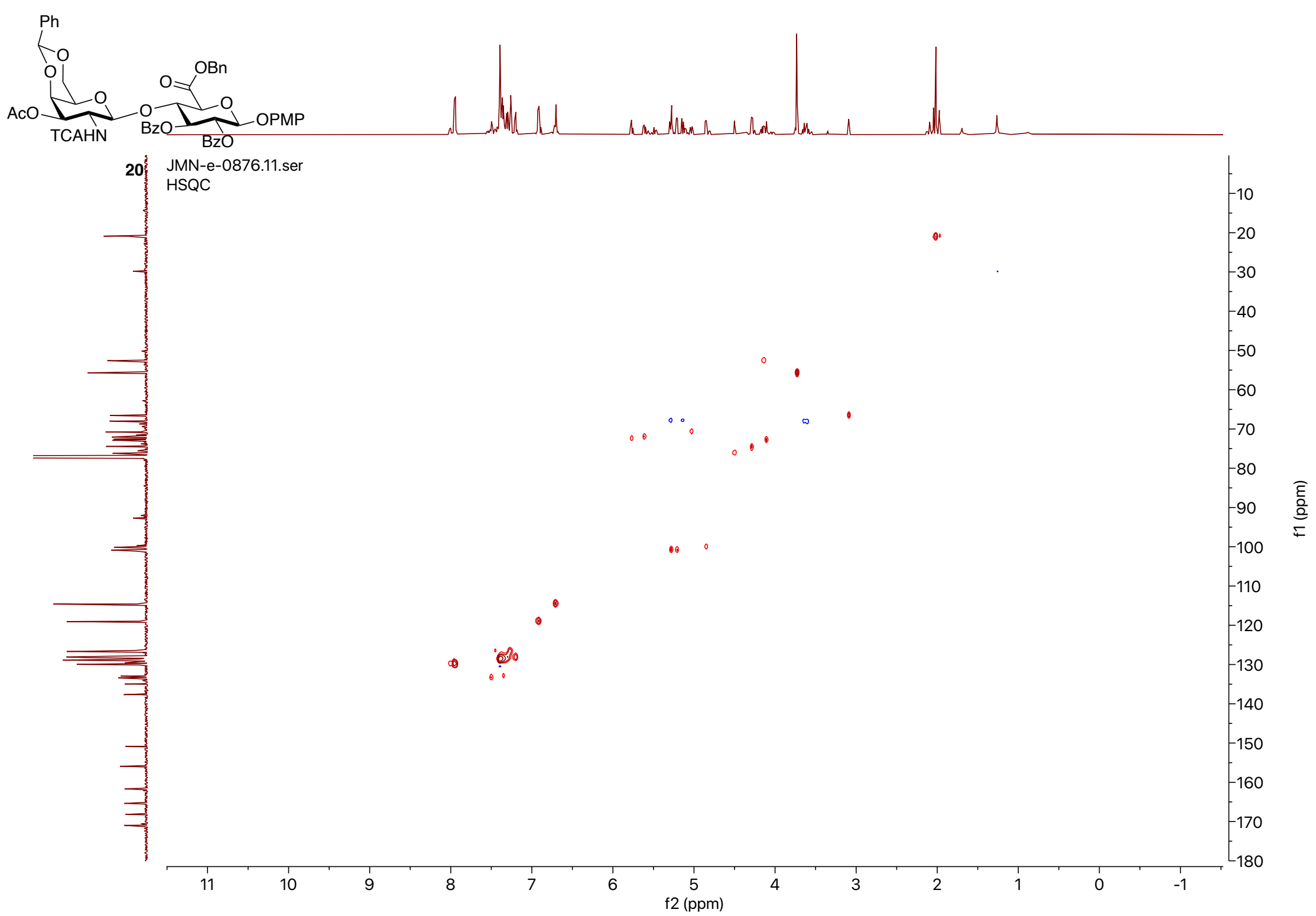


${ }^{1} \mathrm{H}$ NMR (600 MHz, $\mathrm{CDCl}_{3}$ ) of Compound 31

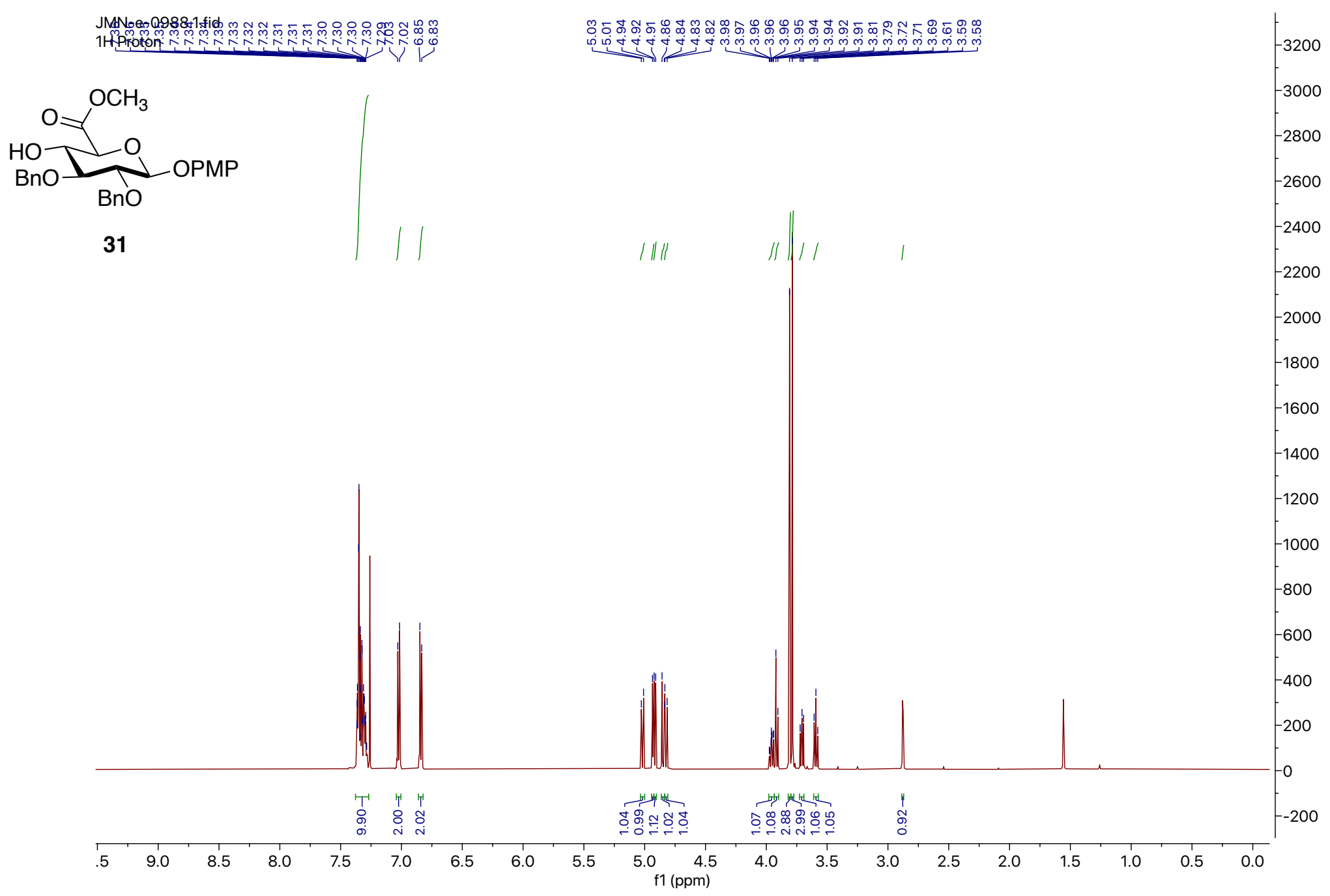


${ }^{13} \mathrm{C}$ NMR (151 MHz, $\left.\mathrm{CDCl}_{3}\right)$ of Compound 31

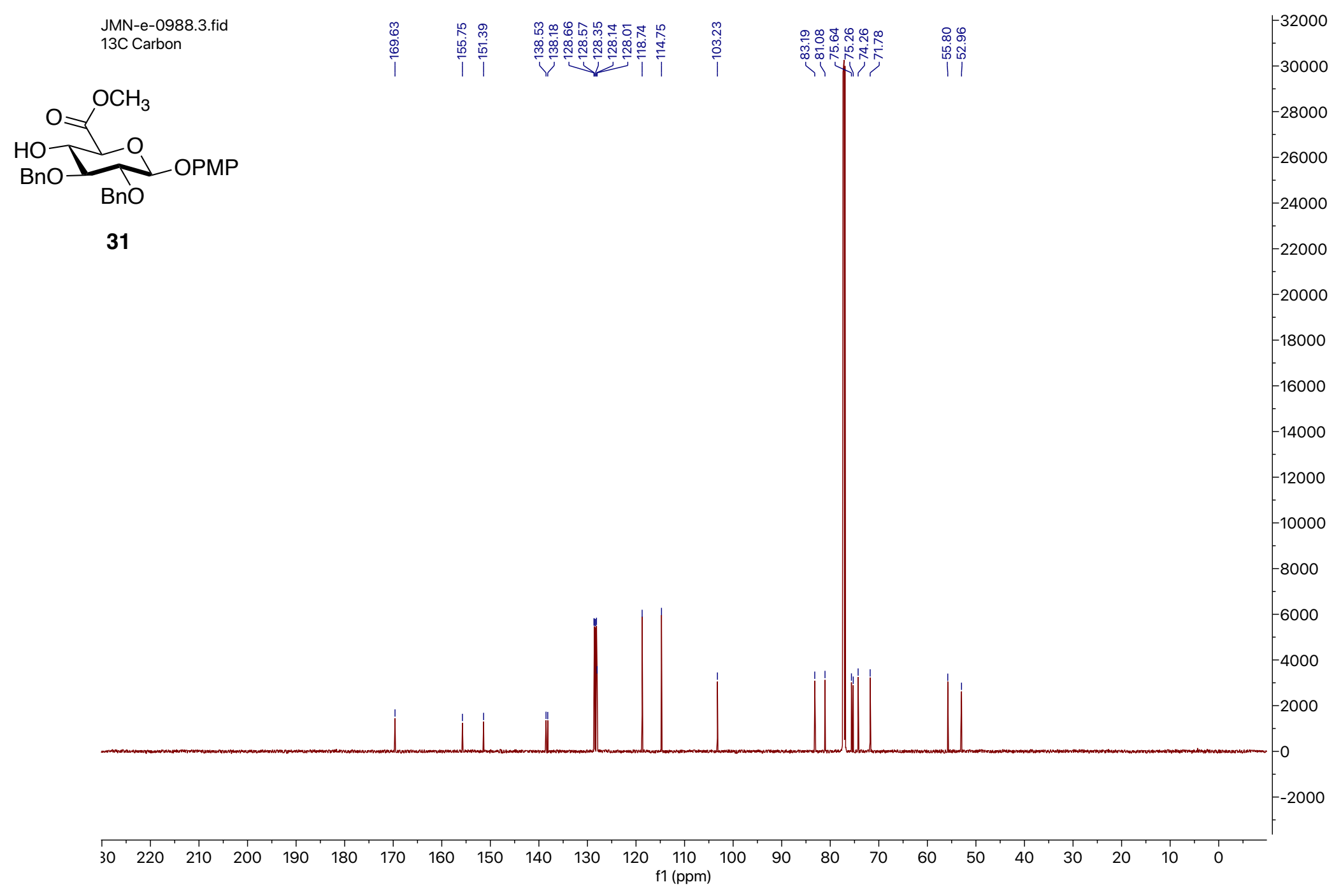


${ }^{1} \mathrm{H}-{ }^{13} \mathrm{C}$ HSQC $\left(\mathrm{CDCl}_{3}\right)$ of Compound 31

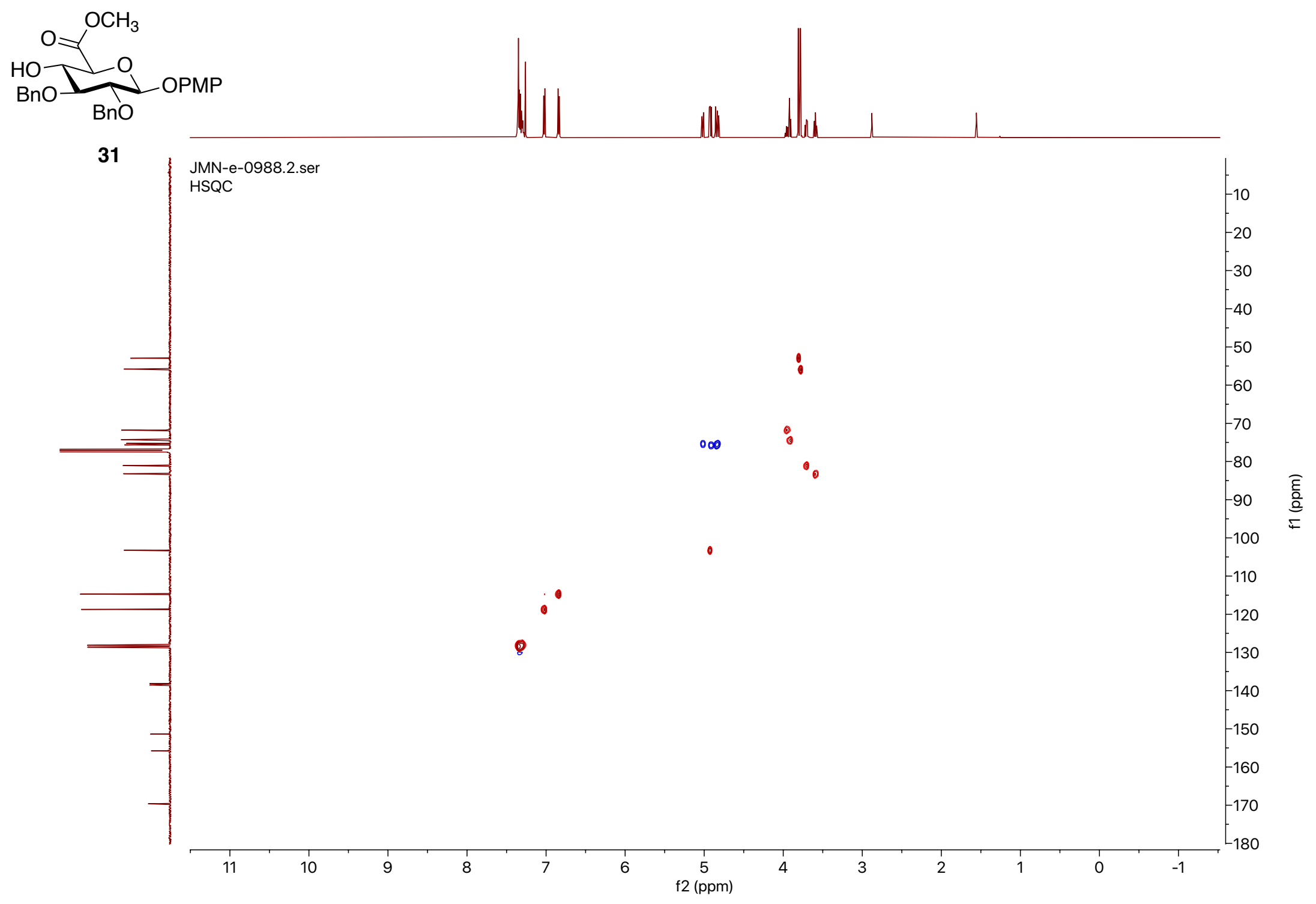


${ }^{1} \mathrm{H}-{ }^{1} \mathrm{H}$ COSY $\left(\mathrm{CDCl}_{3}\right)$ of Compound 31

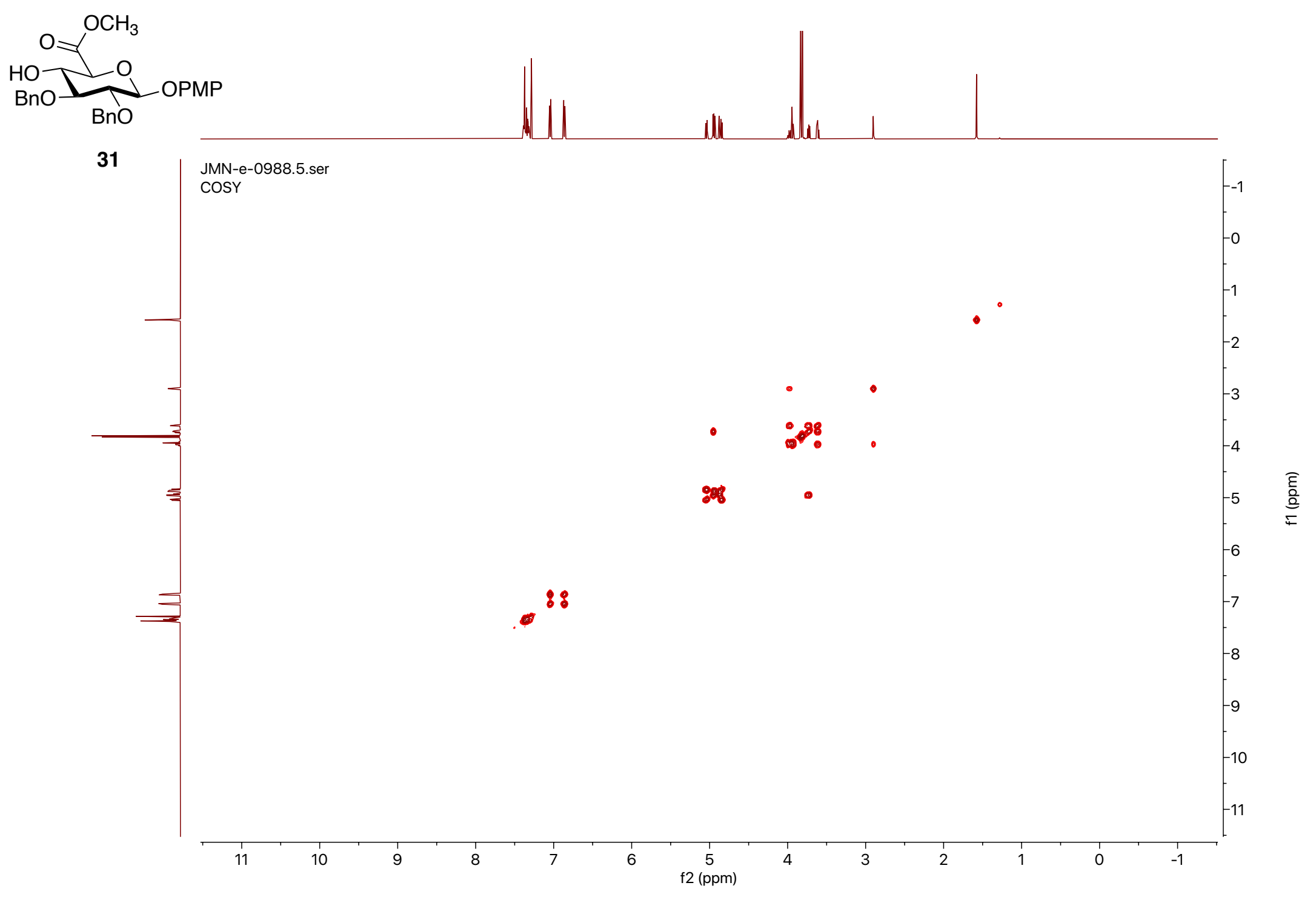


${ }^{1} \mathrm{H}$ NMR (600 MHz, $\mathrm{CDCl}_{3}$ ) of Compound 32

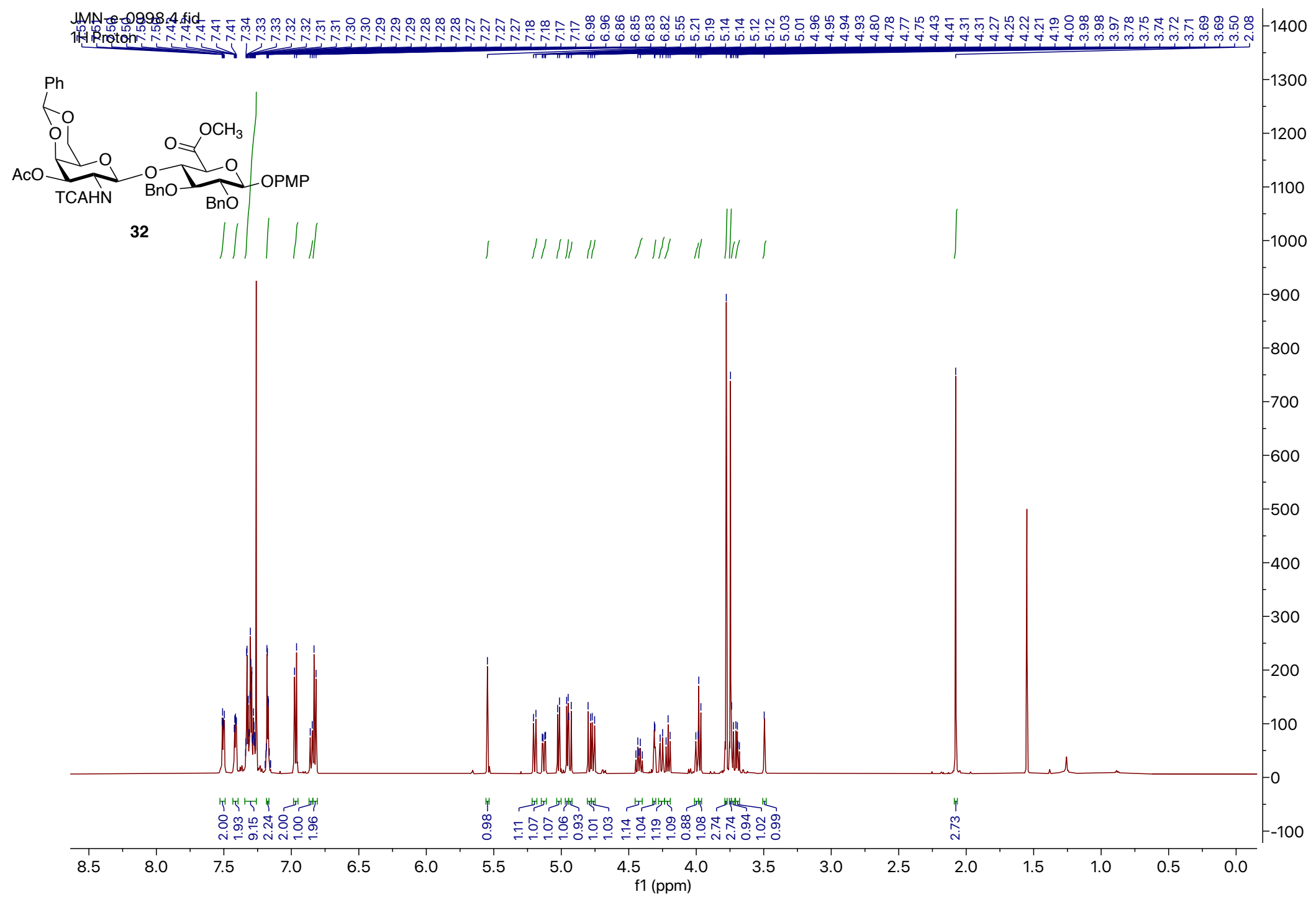


${ }^{13} \mathrm{C}$ NMR (151 MHz, $\left.\mathrm{CDCl}_{3}\right)$ of Compound 32

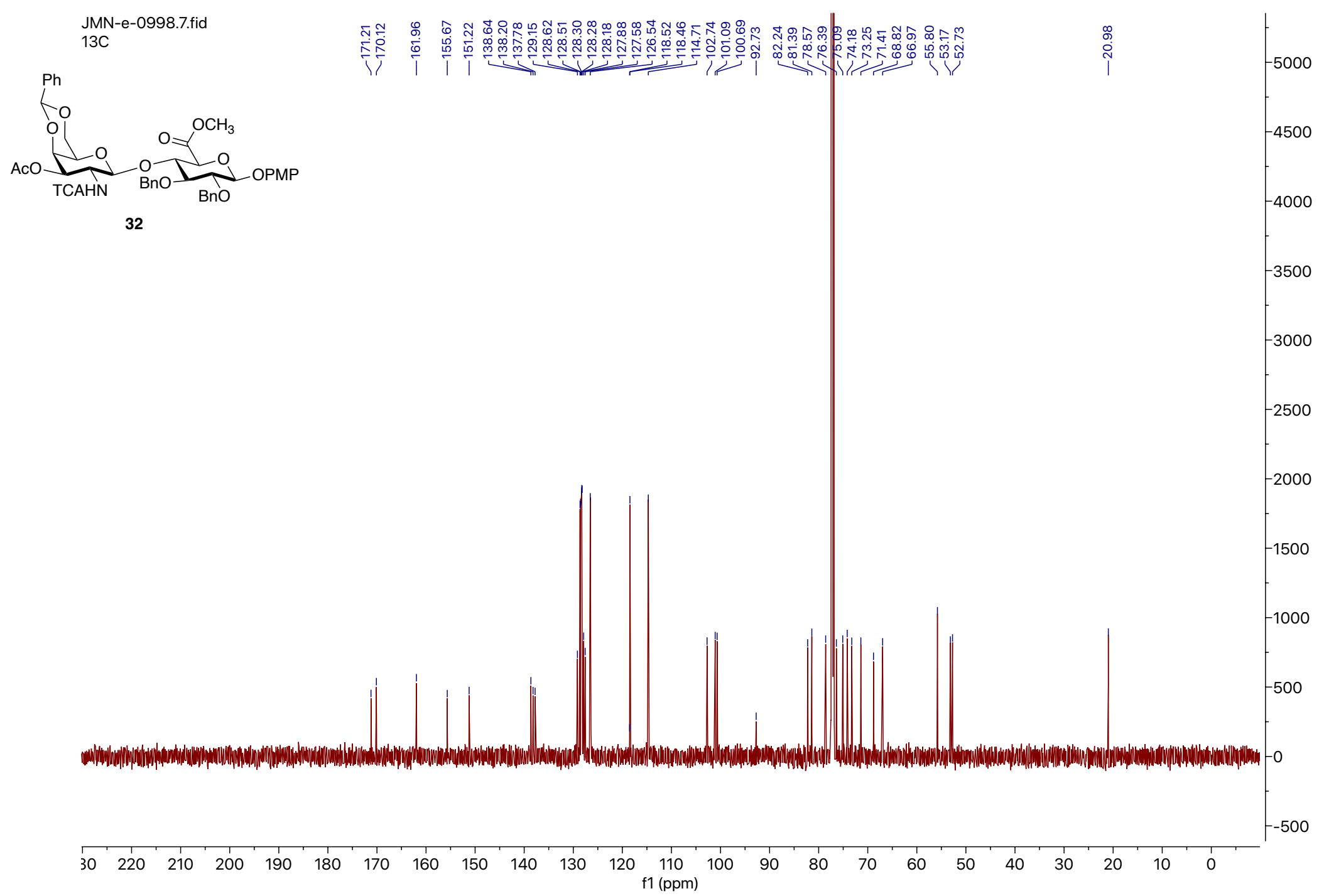


${ }^{1} \mathrm{H}-{ }^{13} \mathrm{C}$ HSQC $\left(\mathrm{CDCl}_{3}\right)$ of $\mathrm{Compound} 32$

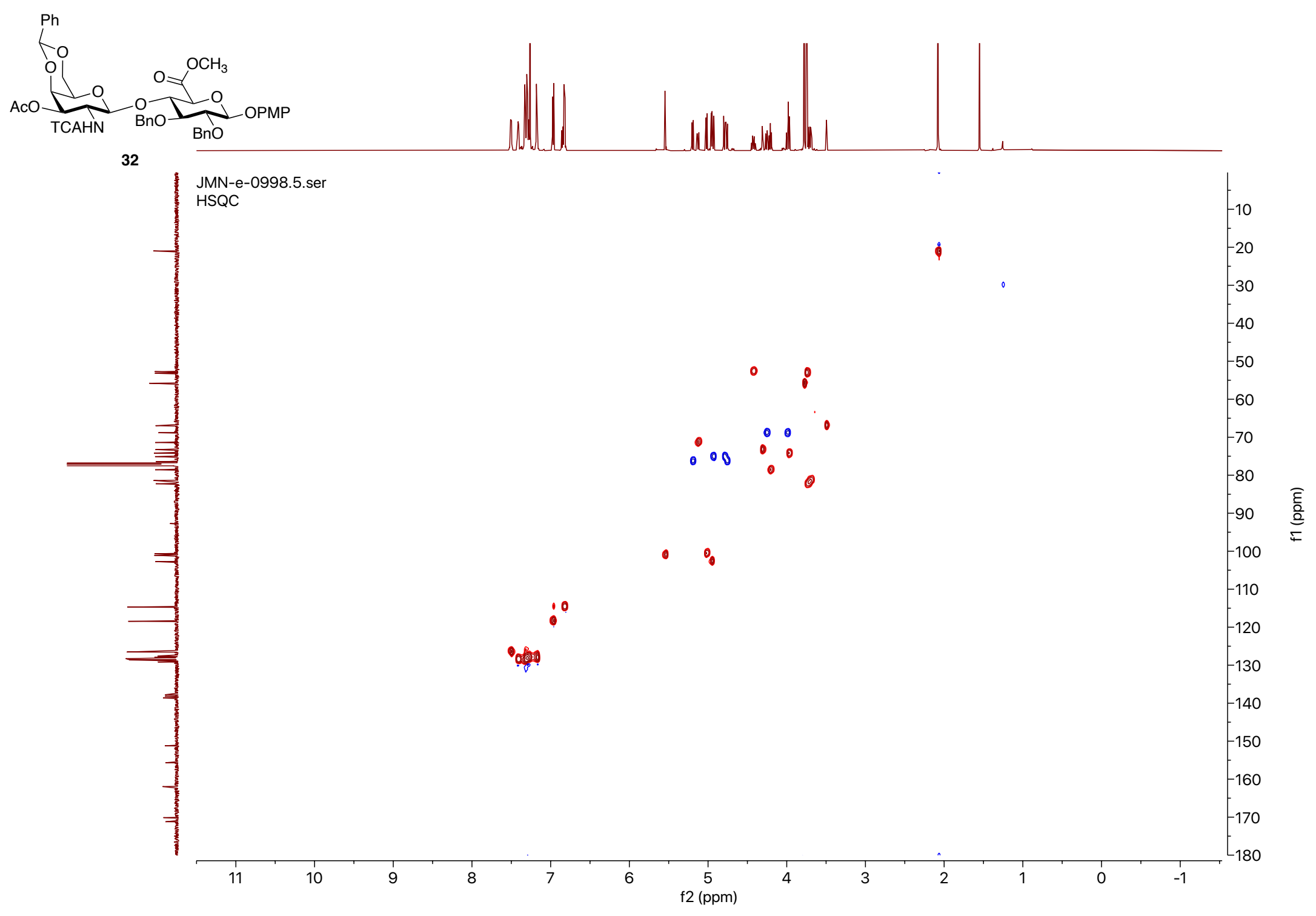


${ }^{1} \mathrm{H}-{ }^{1} \mathrm{H}$ COSY $\left(\mathrm{CDCl}_{3}\right)$ of Compound 32

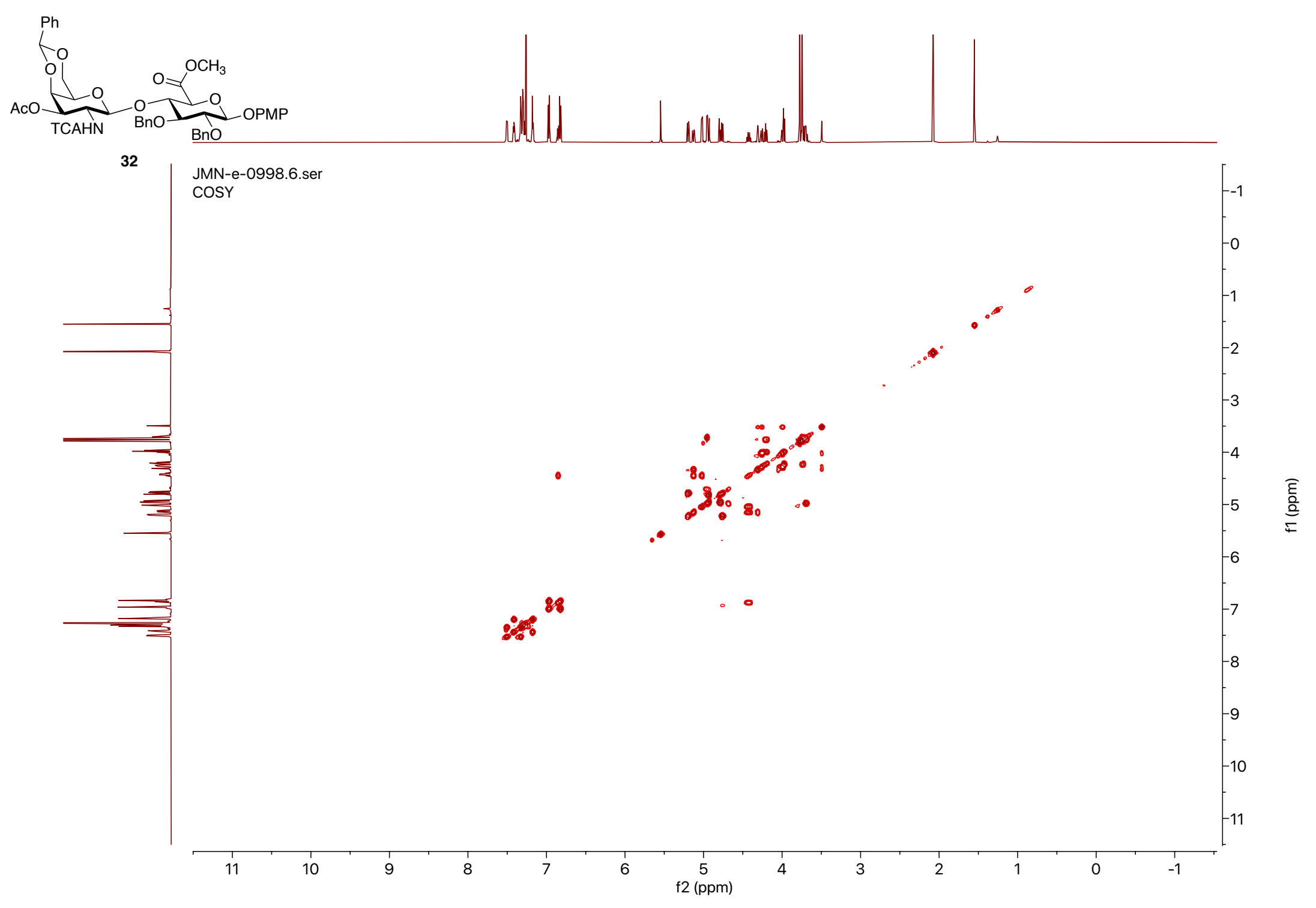


${ }^{1} \mathrm{H}-{ }^{13} \mathrm{C}$ HMBC $\left(\mathrm{CDCl}_{3}\right)$ of Compound 32

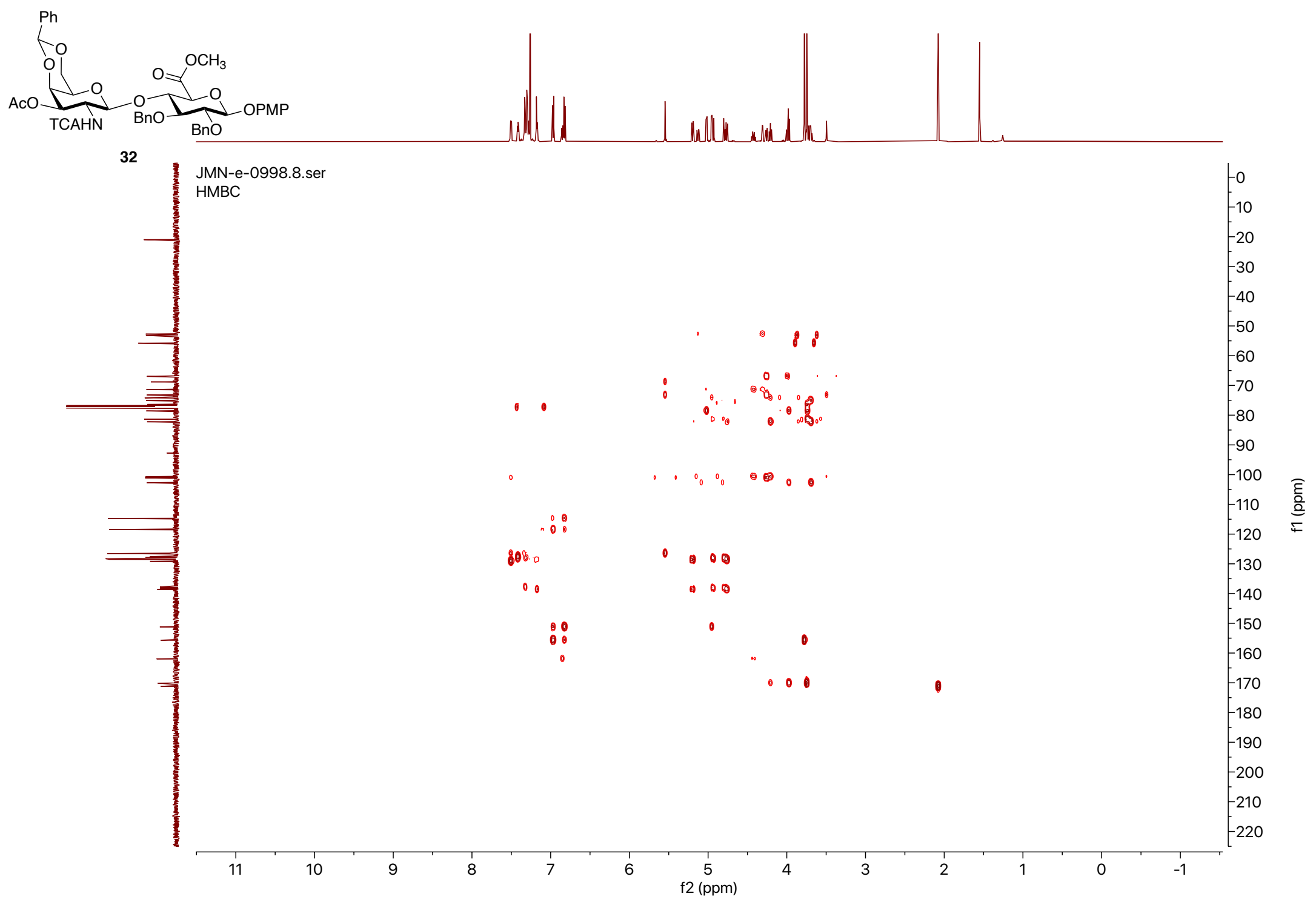


${ }^{1} \mathrm{H}$ NMR (600 MHz, $\mathrm{CDCl}_{3}$ ) of Compound 33

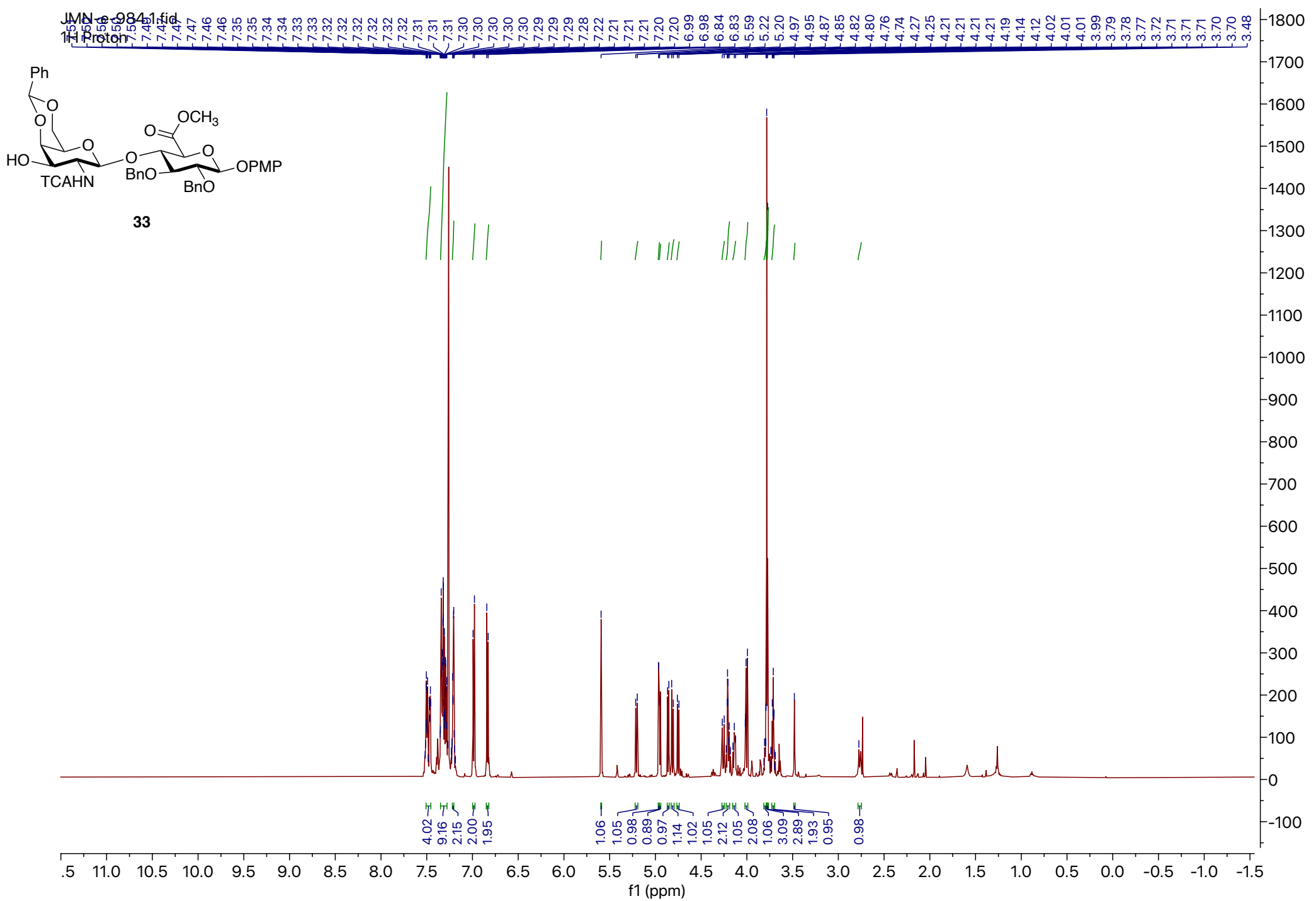


${ }^{13} \mathrm{C}$ NMR (151 MHz, $\left.\mathrm{CDCl}_{3}\right)$ of Compound 33

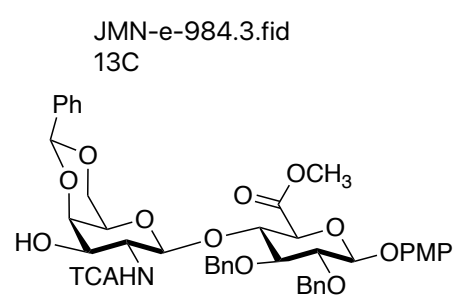

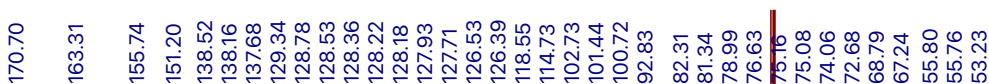

22000

I I I

$-21000$

$-20000$

$-19000$

$-18000$

$-17000$

$-16000$

$-15000$

$-14000$

$-13000$

$-12000$

$-11000$

$-10000$

9000

$-8000$

7000

6000

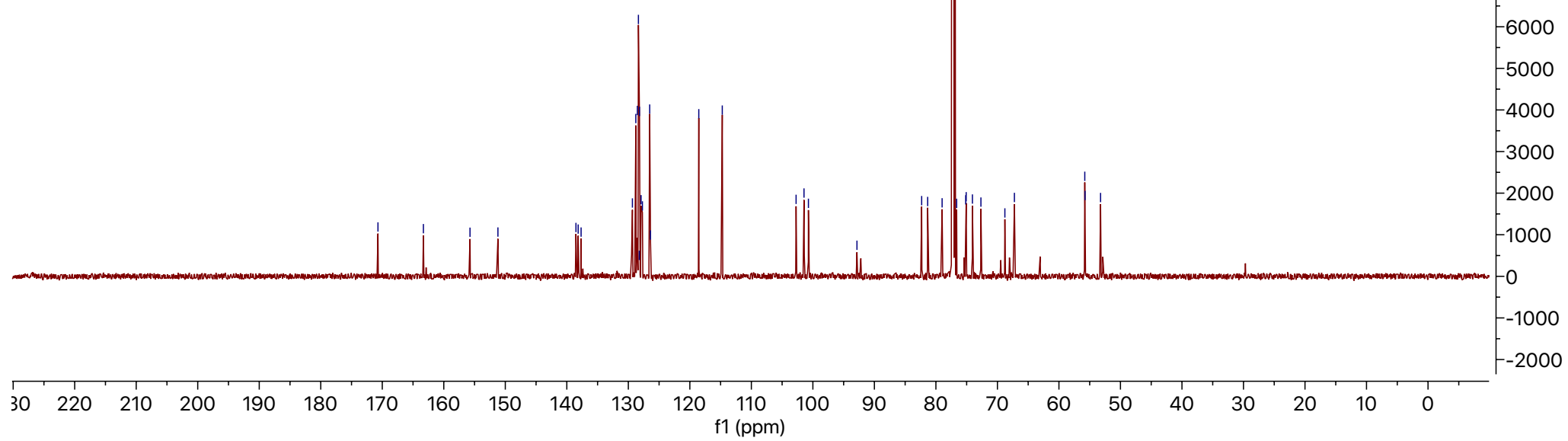


${ }^{1} \mathrm{H}-{ }^{13} \mathrm{C}$ HSQC $\left(\mathrm{CDCl}_{3}\right)$ of $\mathrm{Compound} 33$

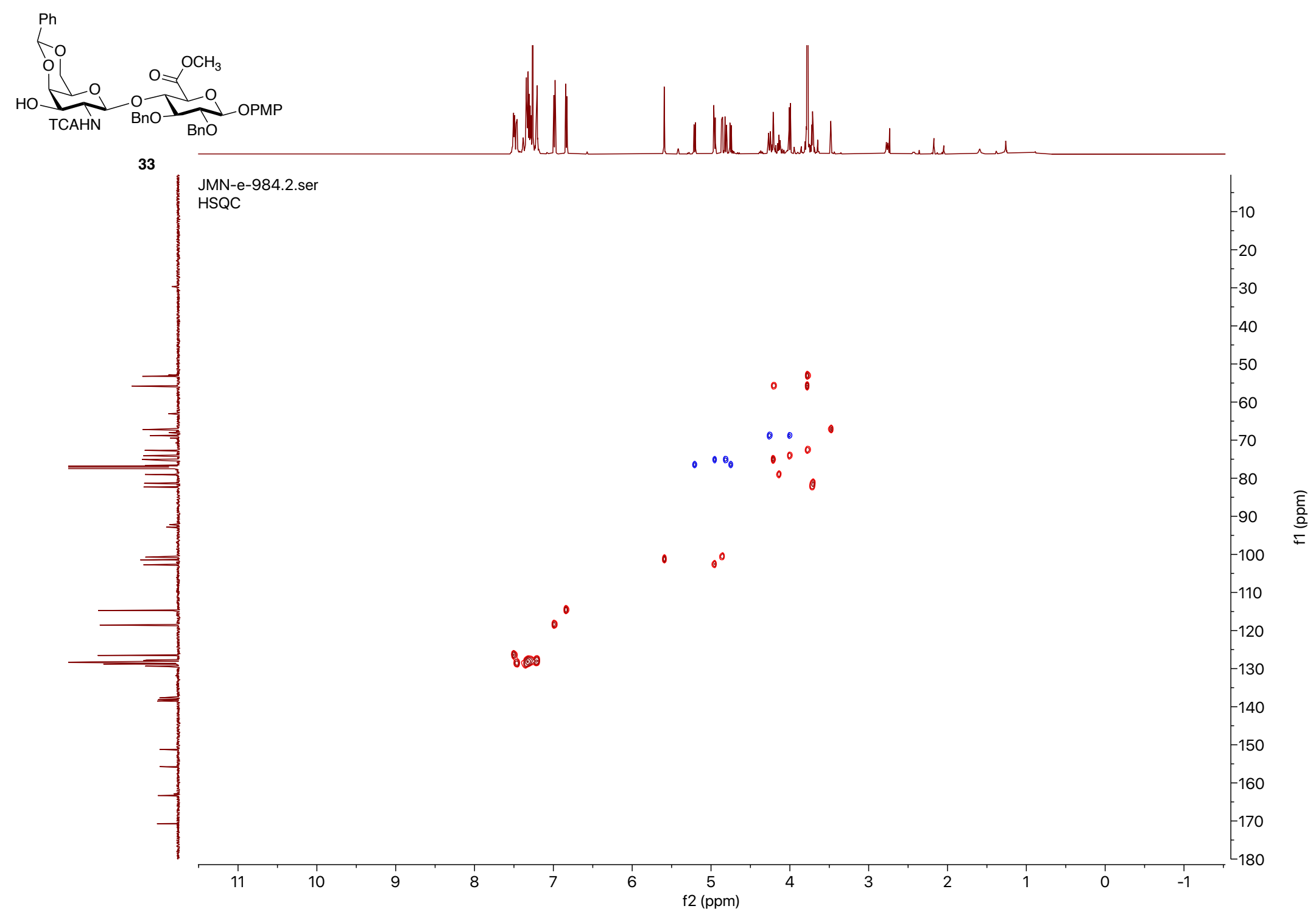


${ }^{1} \mathrm{H}-{ }^{1} \mathrm{H}$ COSY $\left(\mathrm{CDCl}_{3}\right)$ of Compound 33

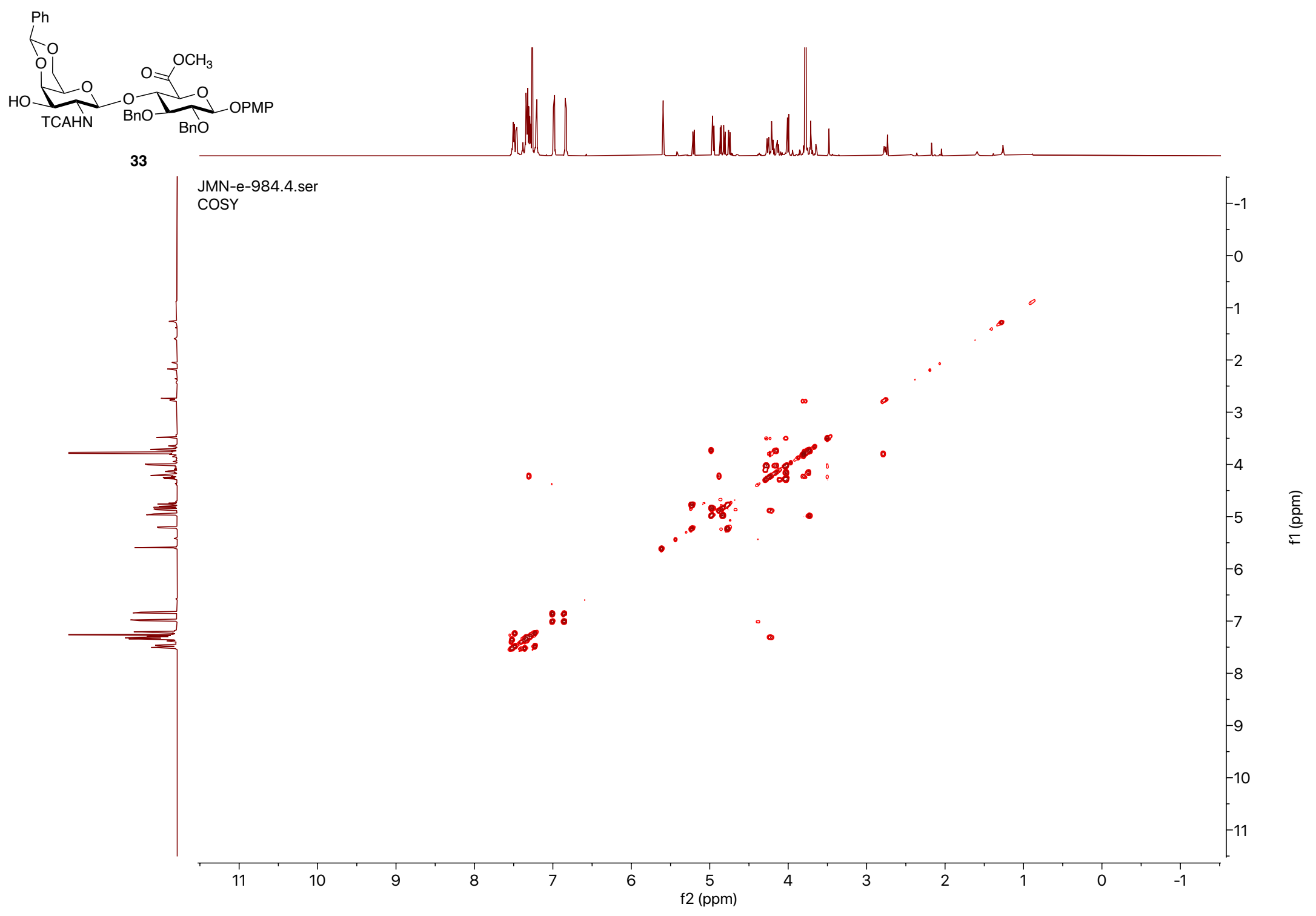


${ }^{1} \mathrm{H}-{ }^{13} \mathrm{C}$ HMBC $\left(\mathrm{CDCl}_{3}\right)$ of Compound 33
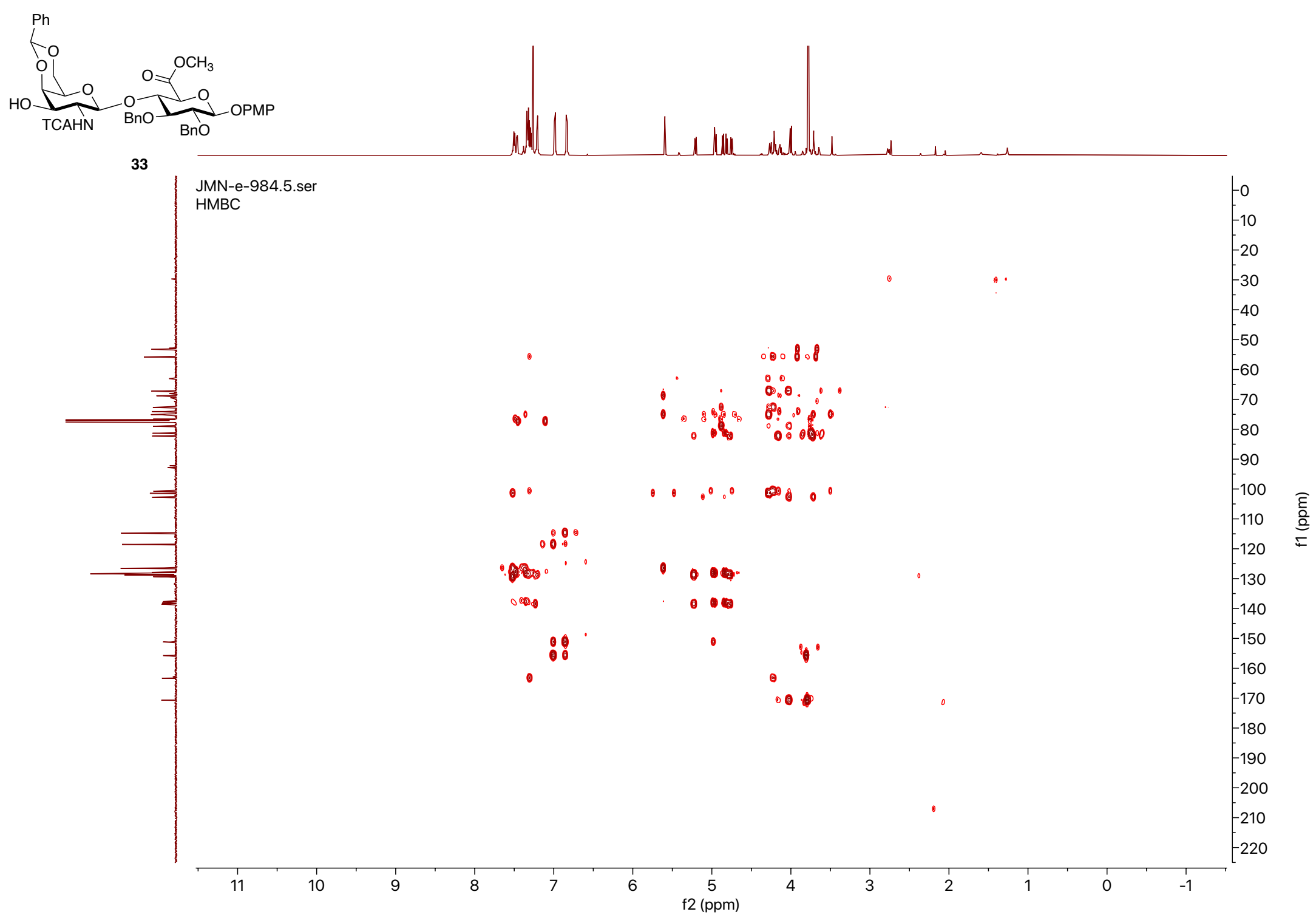
${ }^{1} \mathrm{H}$ NMR (600 MHz, $\left.\mathrm{CDCl}_{3}\right)$ of Compound 34

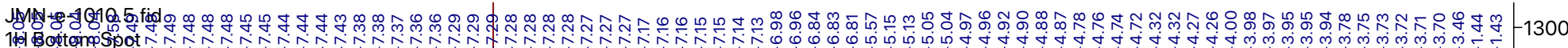

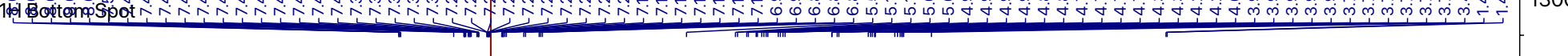

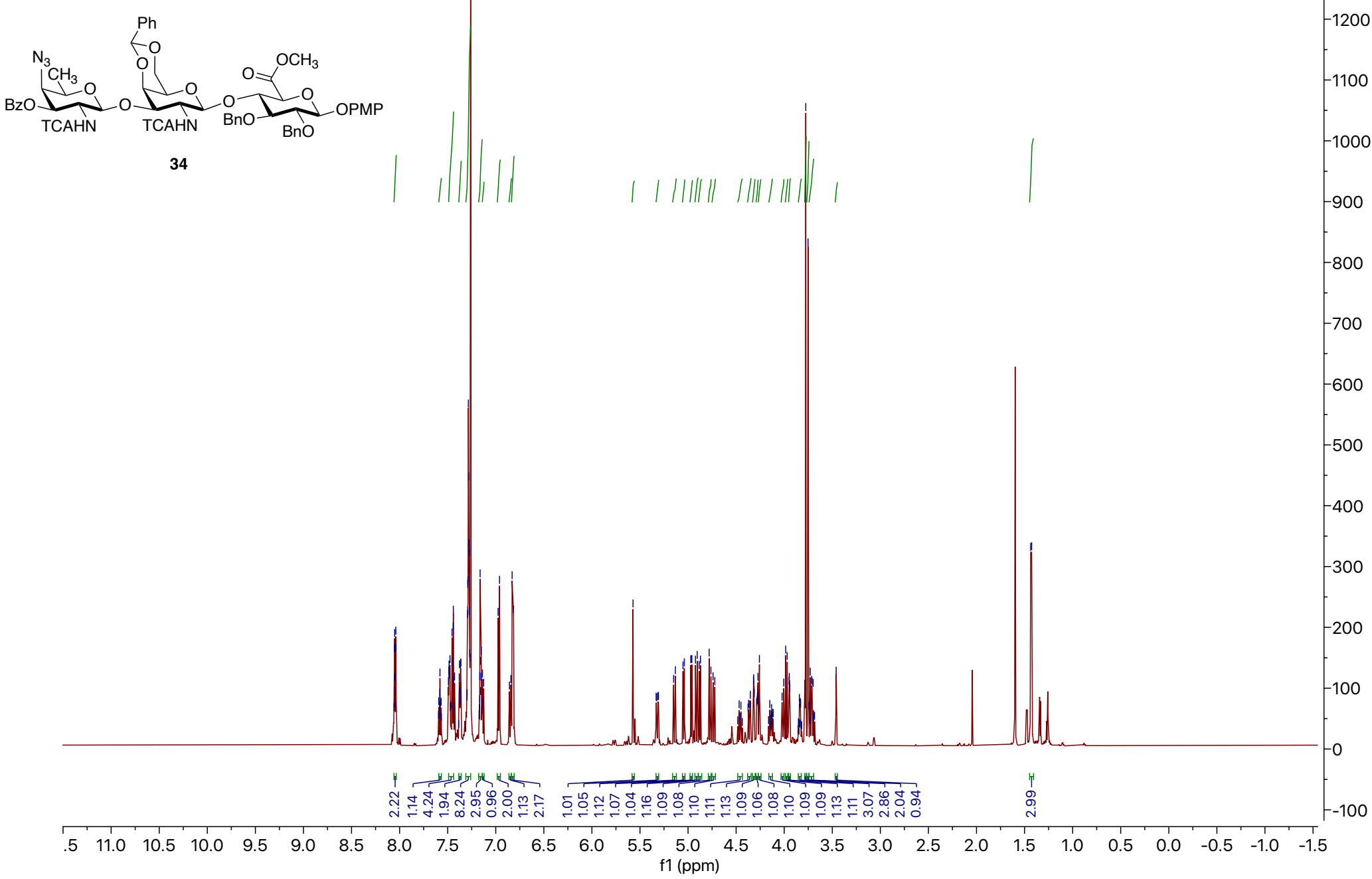


${ }^{13} \mathrm{C}$ NMR (151 MHz, $\left.\mathrm{CDCl}_{3}\right)$ of Compound 34

JMN-e-1010.9.fid

13C Bottom Spot

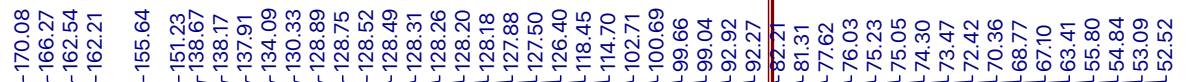

TCAHN

34

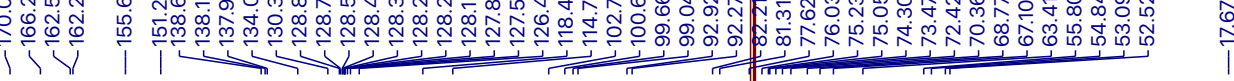

$-9000$

$-8000$

$-7000$

$-6000$

$-5000$

$-4000$

$-3000$

2000

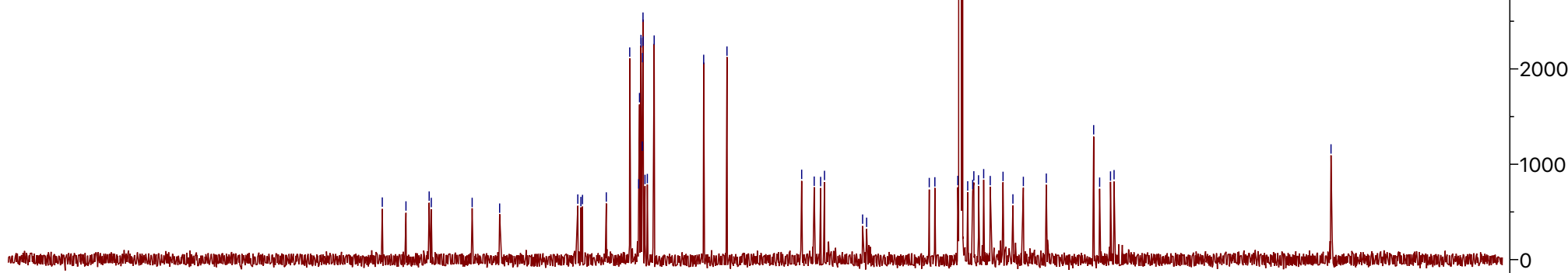

$120110 \quad 100$

$90 \quad 80 \quad 70$

60

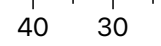

20

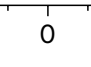

- 
${ }^{1} \mathrm{H}-{ }^{13} \mathrm{C}$ HSQC $\left(\mathrm{CDCl}_{3}\right)$ of Compound 34

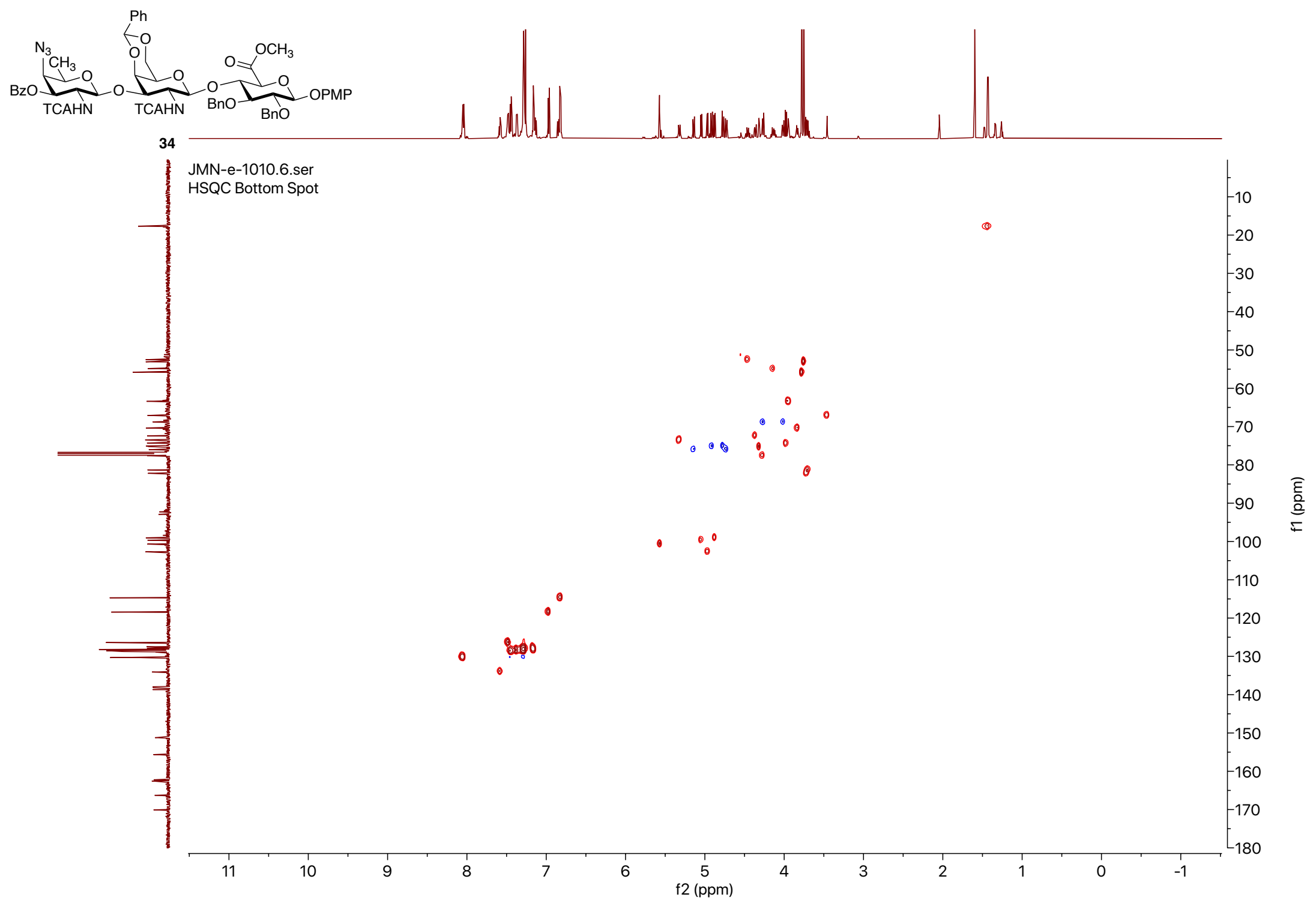


${ }^{1} \mathrm{H}-{ }^{1} \mathrm{H}$ COSY $\left(\mathrm{CDCl}_{3}\right)$ of Compound 34

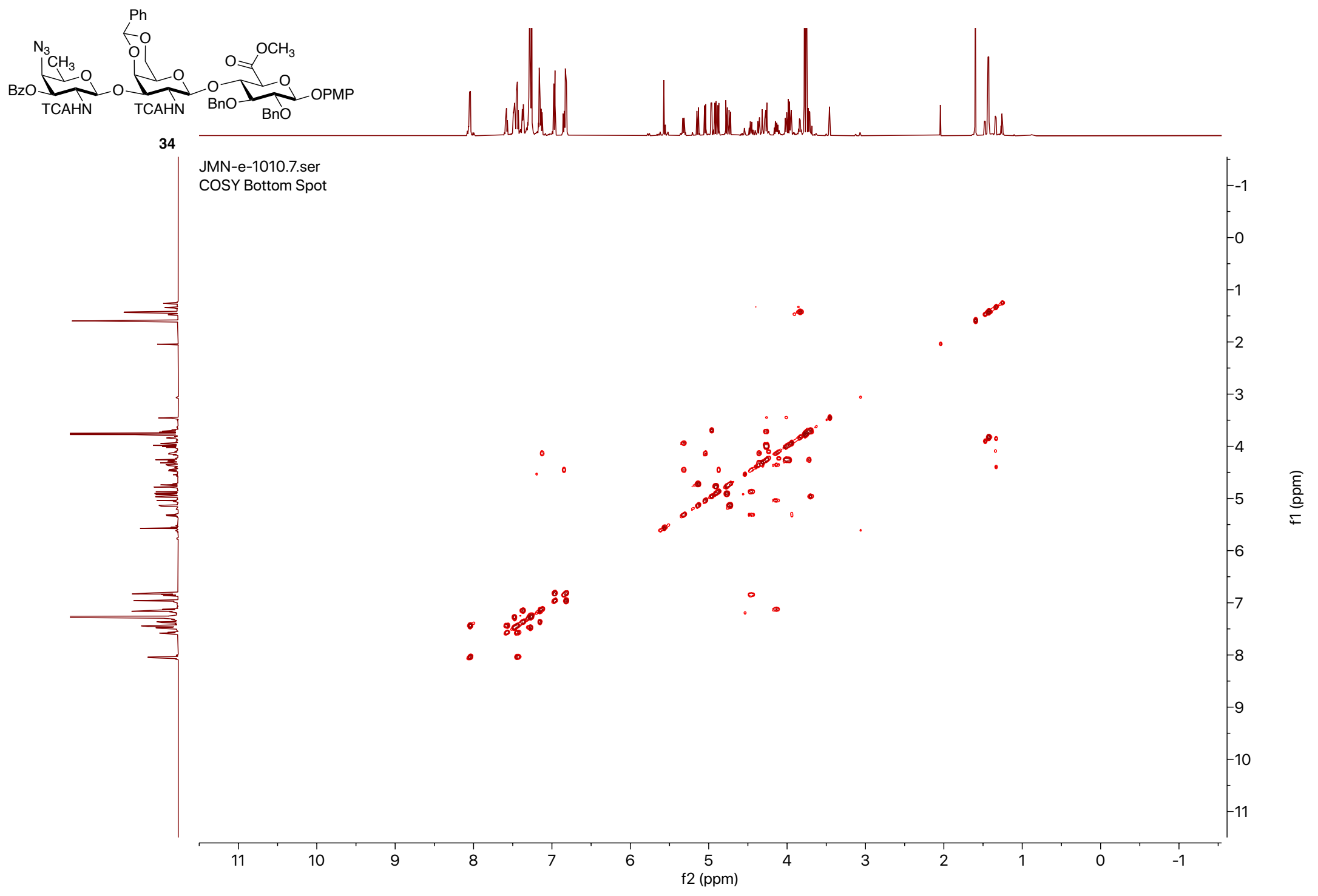


${ }^{1} \mathrm{H}-{ }^{13} \mathrm{C}$ HMBC $\left(\mathrm{CDCl}_{3}\right)$ of Compound 34

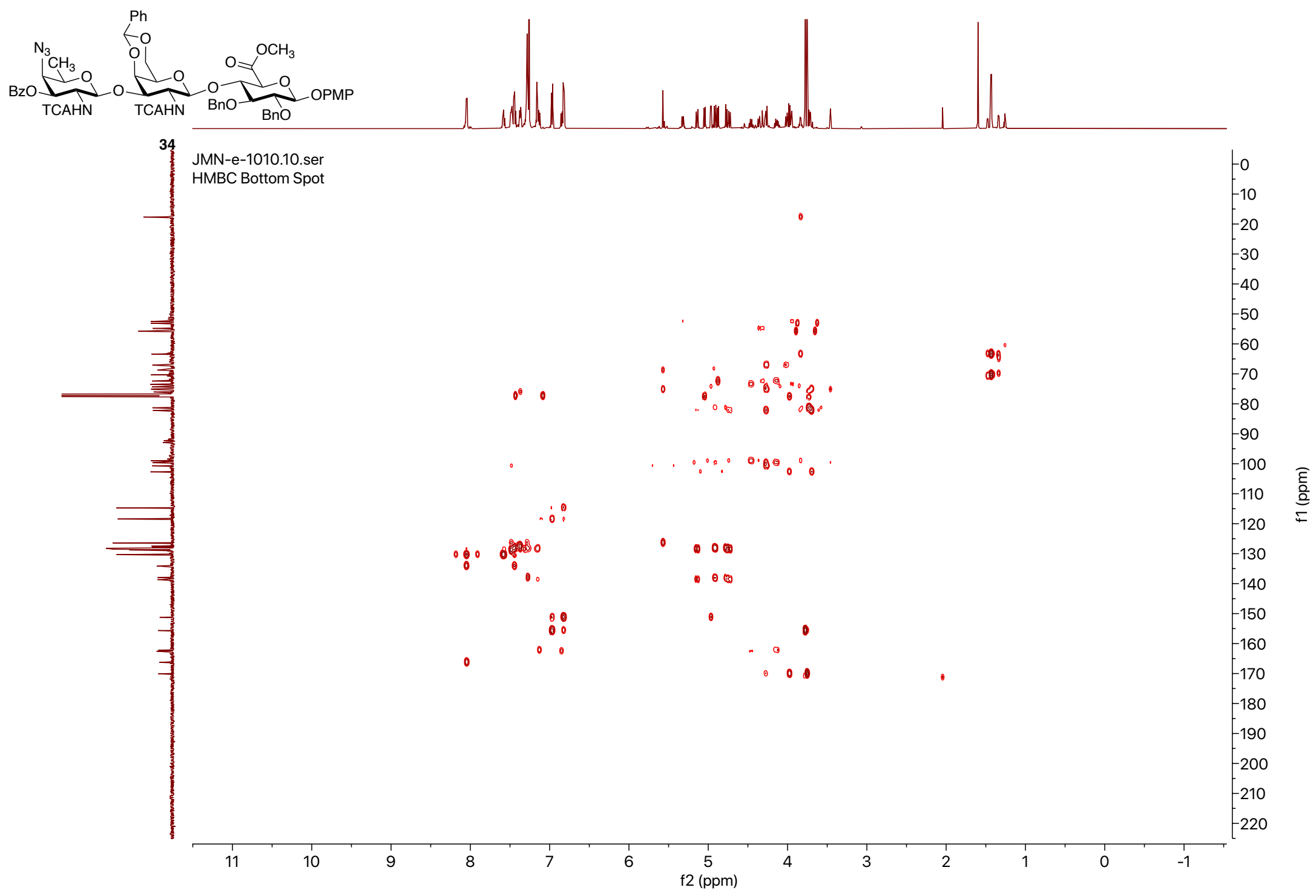


${ }^{1} \mathrm{H}$ NMR (600 MHz, $\mathrm{CDCl}_{3}$ ) of Compound 35

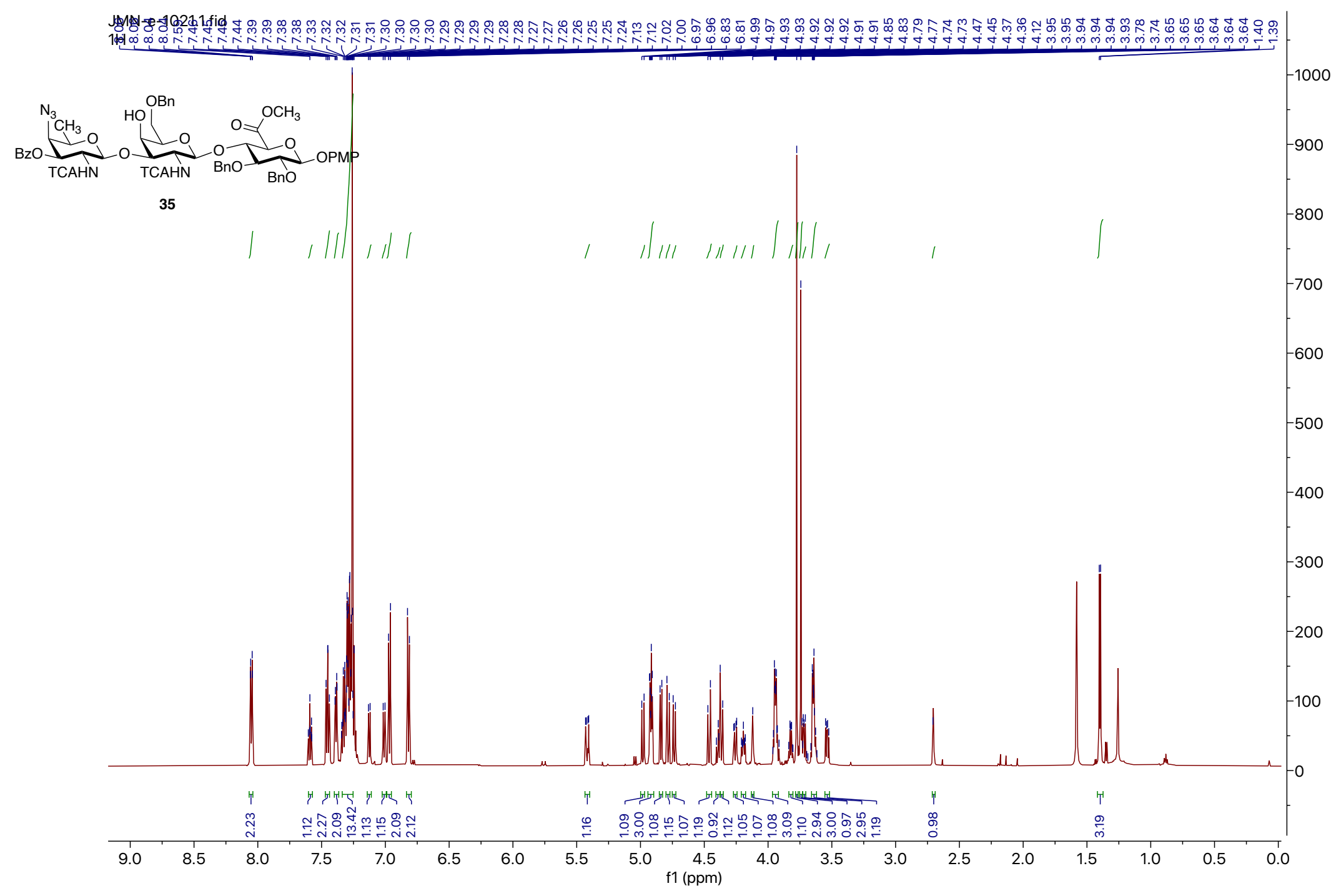


${ }^{13} \mathrm{C}$ NMR (151 MHz, $\left.\mathrm{CDCl}_{3}\right)$ of Compound 35

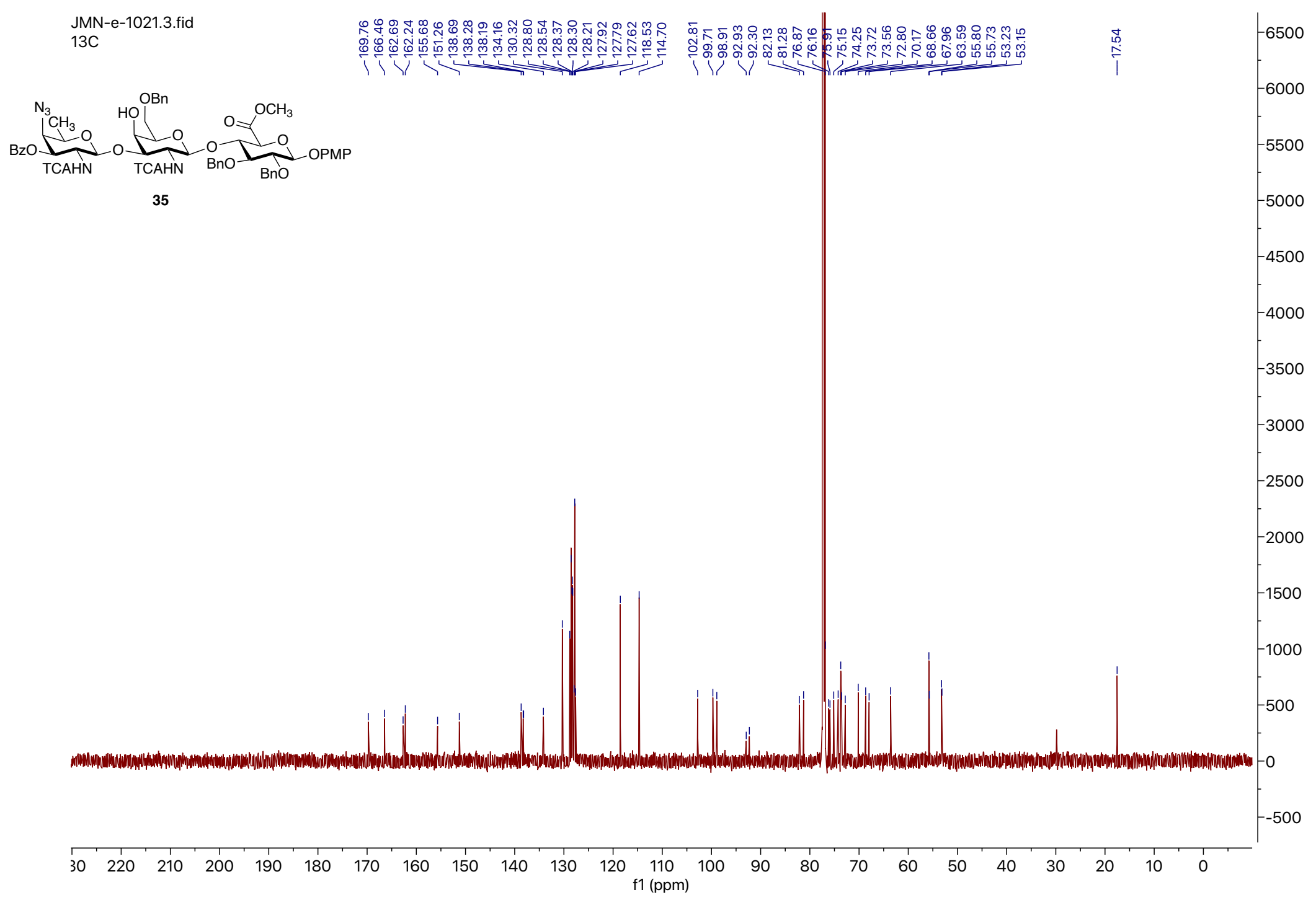


${ }^{1} \mathrm{H}-{ }^{13} \mathrm{C}$ HSQC $\left(\mathrm{CDCl}_{3}\right)$ of $\mathrm{Compound} 35$

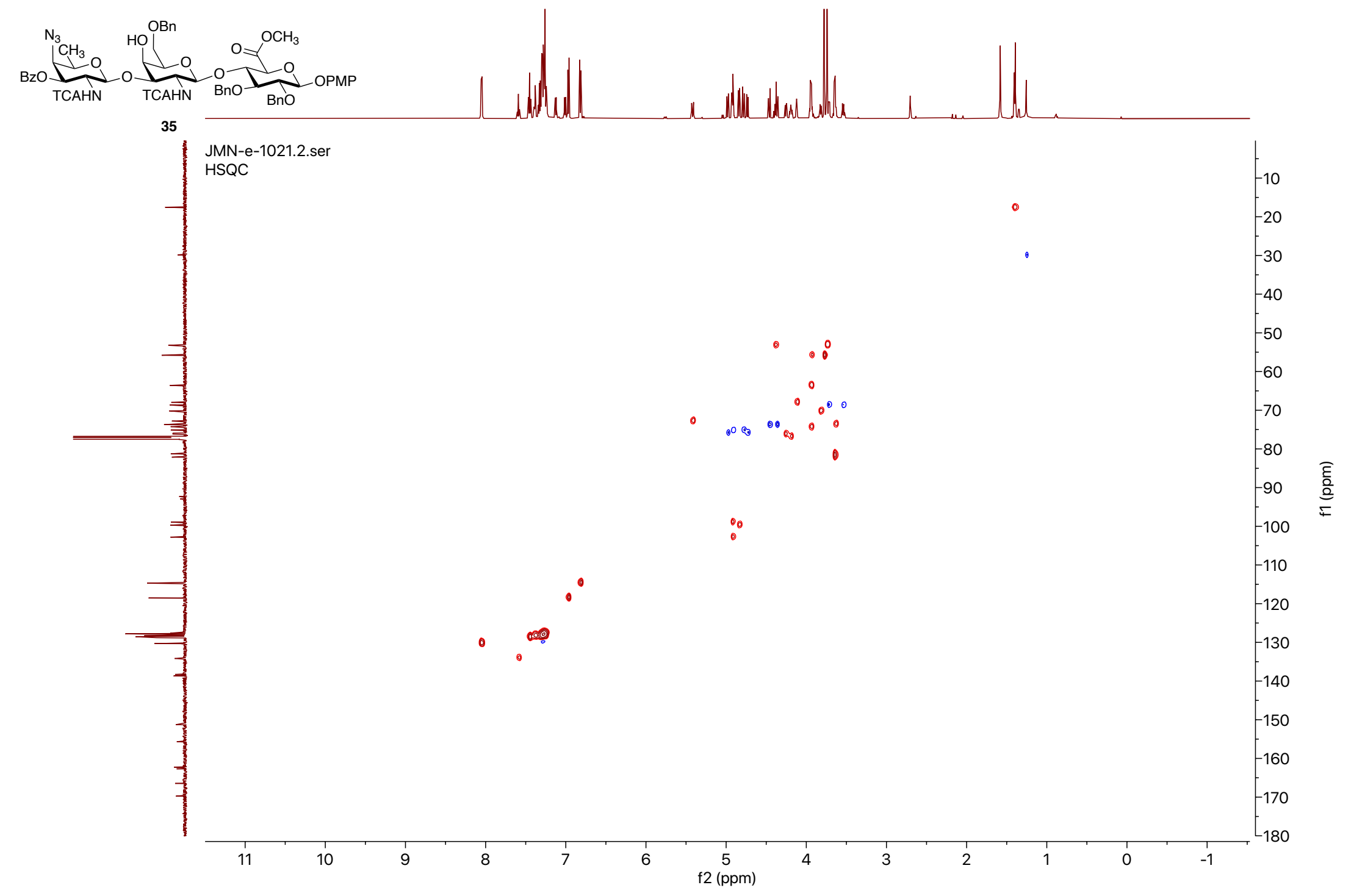


${ }^{1} \mathrm{H}-{ }^{1} \mathrm{H}$ COSY $\left(\mathrm{CDCl}_{3}\right)$ of Compound 35

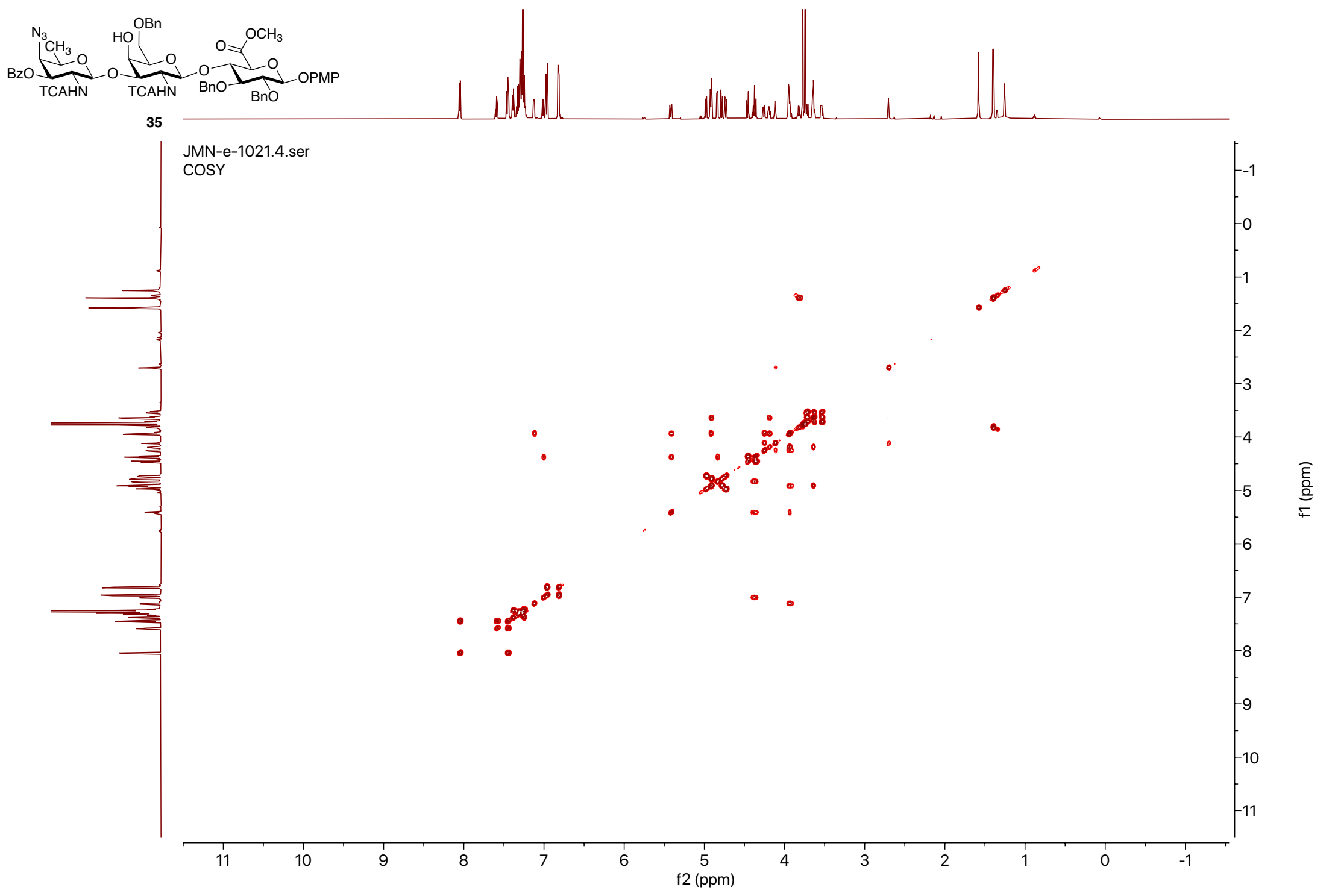


${ }^{1} \mathrm{H}-{ }^{13} \mathrm{C}$ HMBC $\left(\mathrm{CDCl}_{3}\right)$ of Compound 35

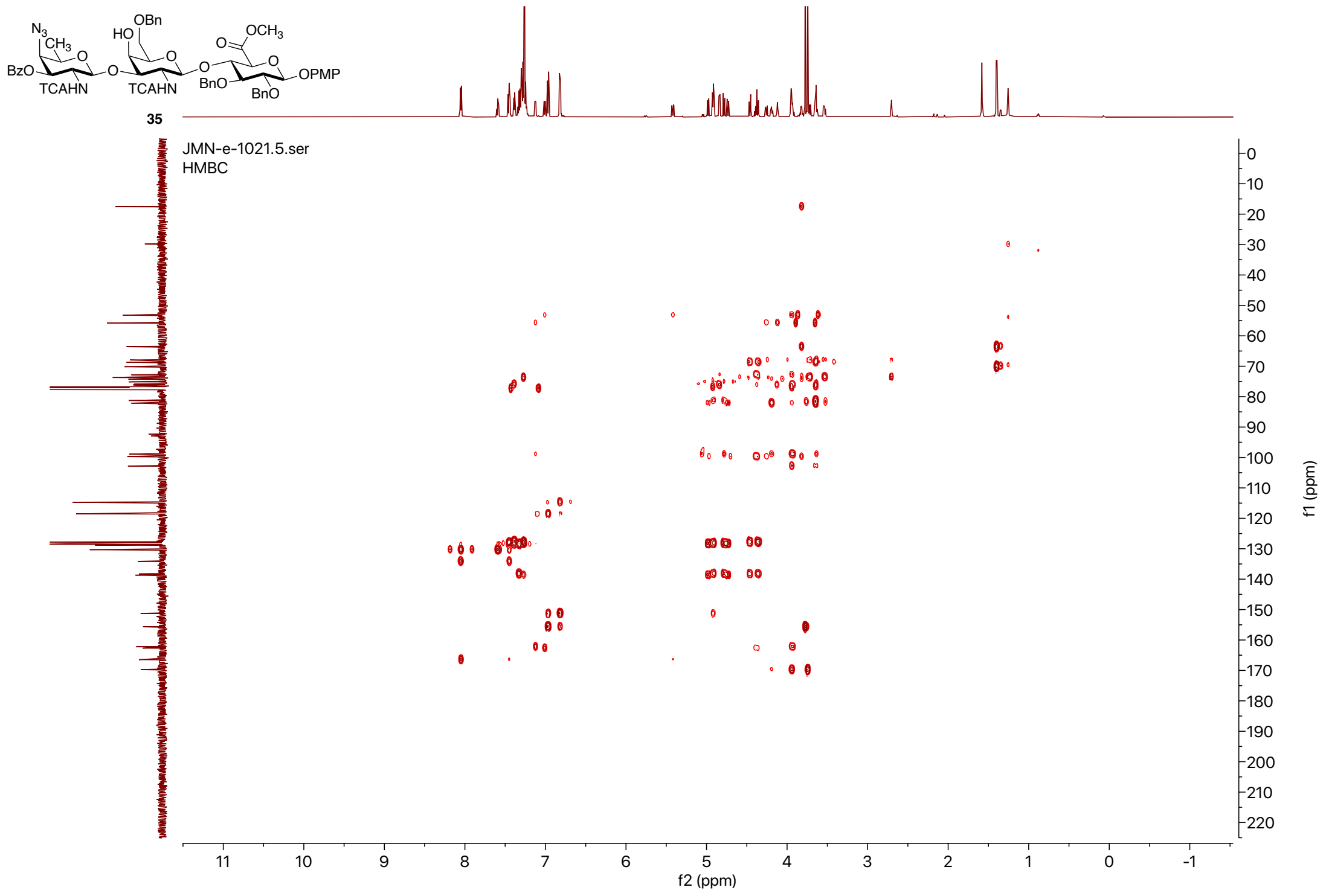


${ }^{1} \mathrm{H}$ NMR (600 MHz, $\left.\mathrm{CDCl}_{3}\right)$ of Compound 36

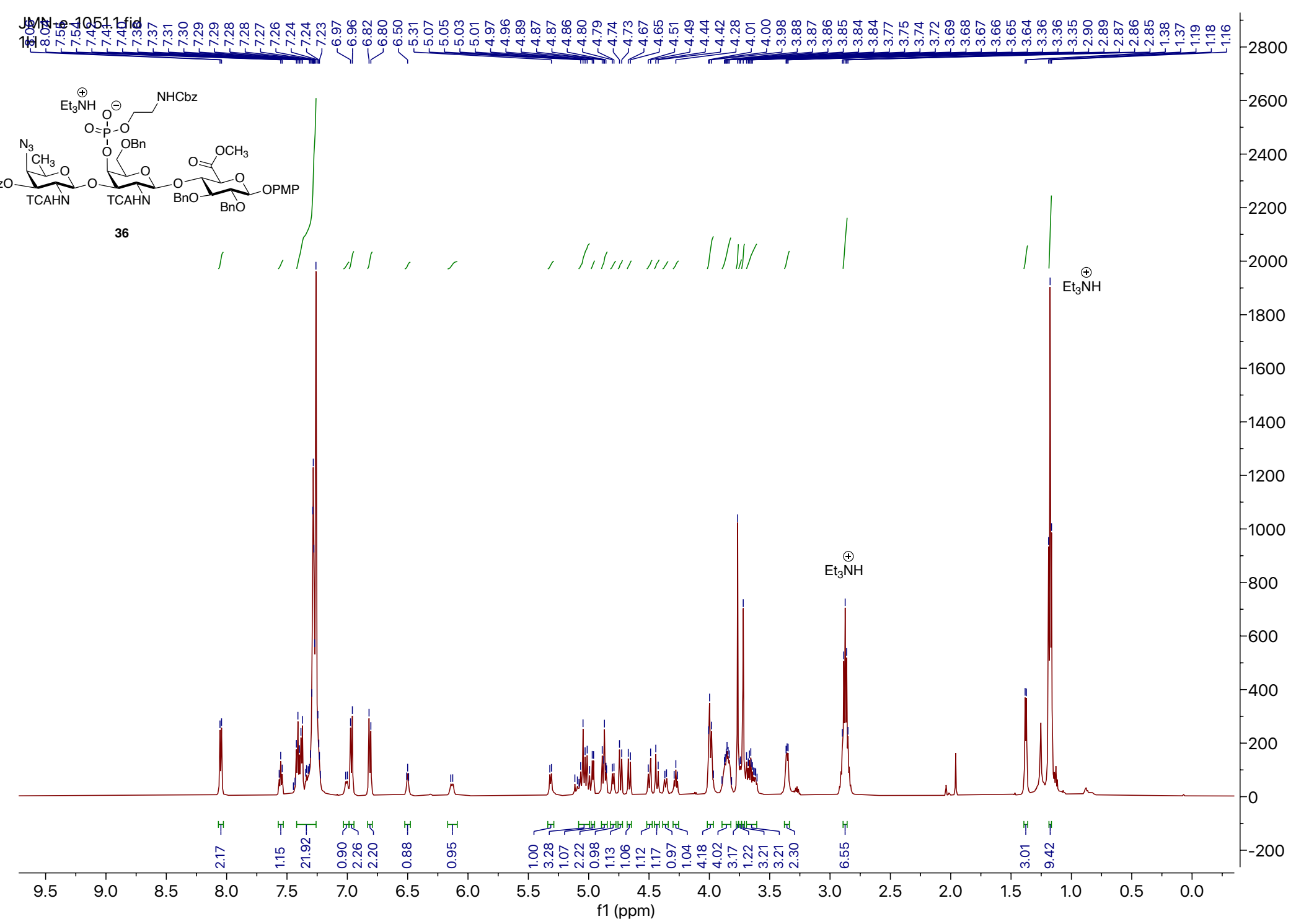


${ }^{13} \mathrm{C}$ NMR (151 MHz, $\left.\mathrm{CDCl}_{3}\right)$ of Compound 36

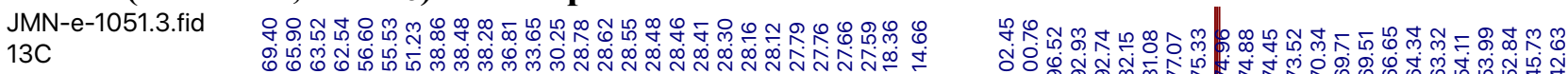

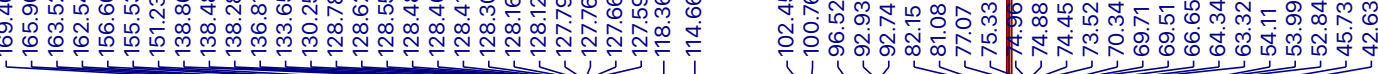

$\stackrel{\oplus}{\stackrel{\oplus}{N}} \mathrm{Et}_{3} \odot \underbrace{\mathrm{NHCbz}}$

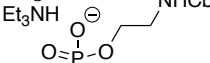

$\mathrm{N}^{\mathrm{N}} \quad \mathrm{O}$

$\mathrm{O}=\mathrm{OCH}_{3}$

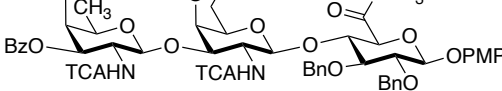

36

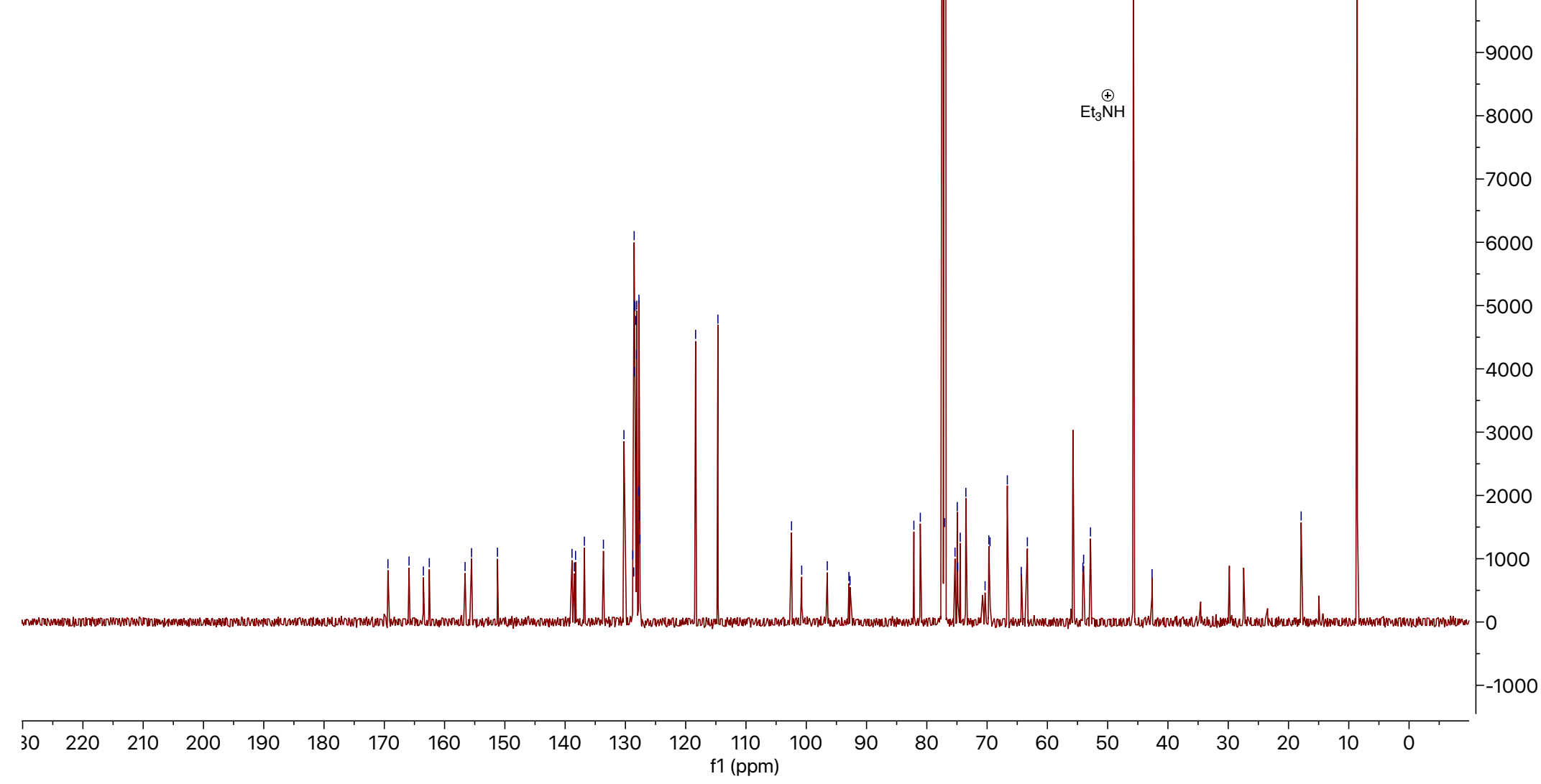


${ }^{1} \mathrm{H}-{ }^{13} \mathrm{C}$ HSQC $\left(\mathrm{CDCl}_{3}\right)$ of $\mathrm{Compound} 36$

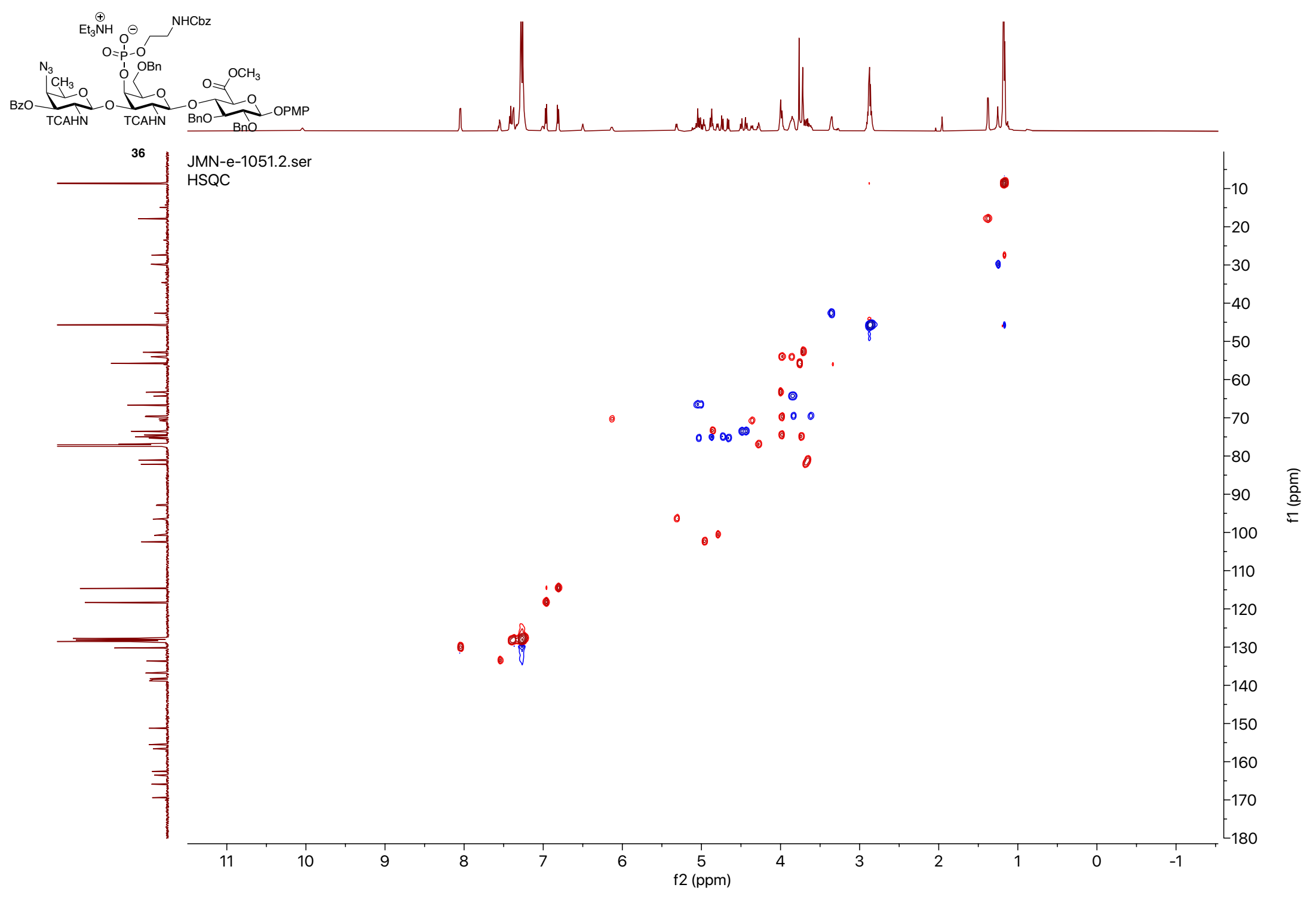


${ }^{1} \mathrm{H}-{ }^{1} \mathrm{H}$ COSY $\left(\mathrm{CDCl}_{3}\right)$ of Compound 36

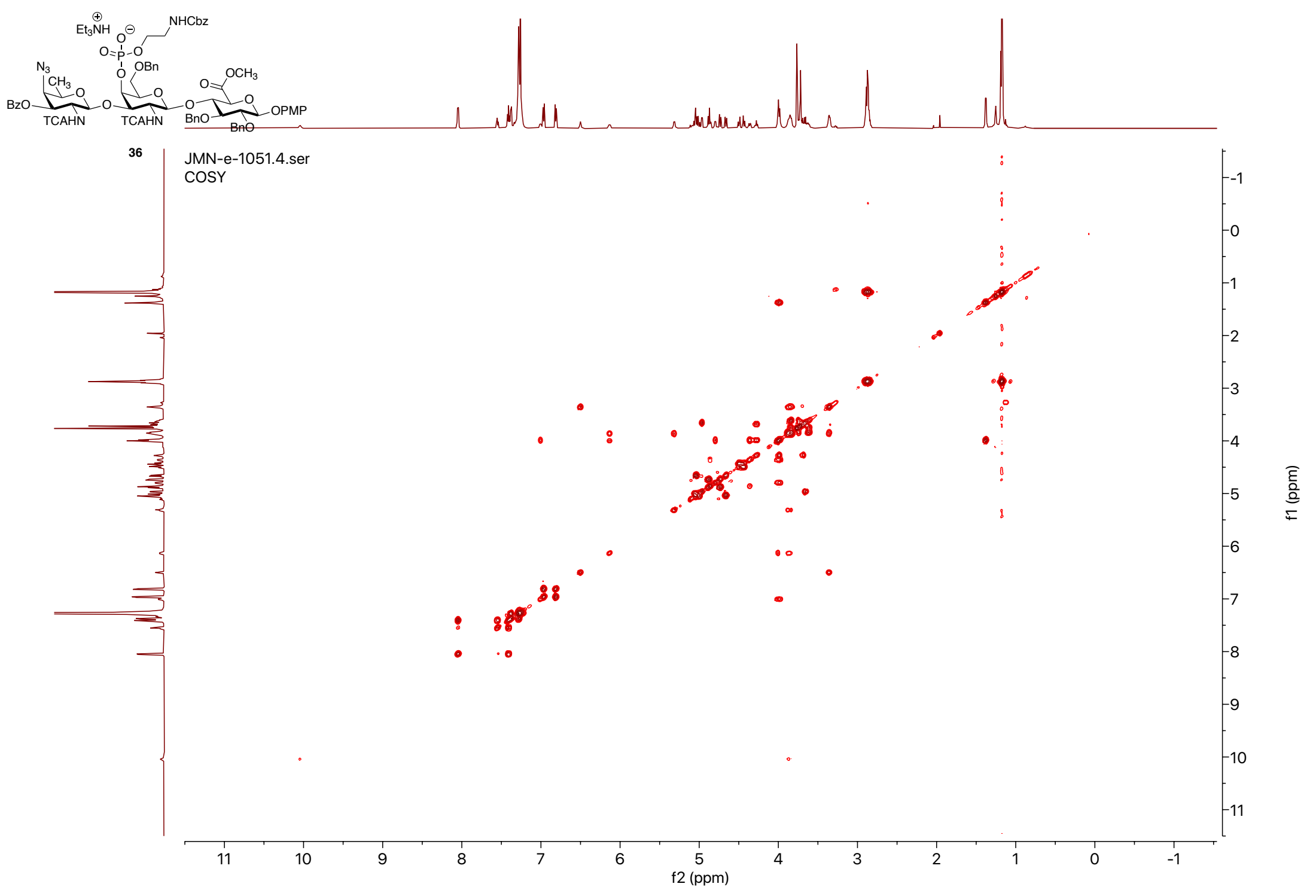


${ }^{1} \mathrm{H}-{ }^{13} \mathrm{C}$ HMBC $\left(\mathrm{CDCl}_{3}\right)$ of Compound 36

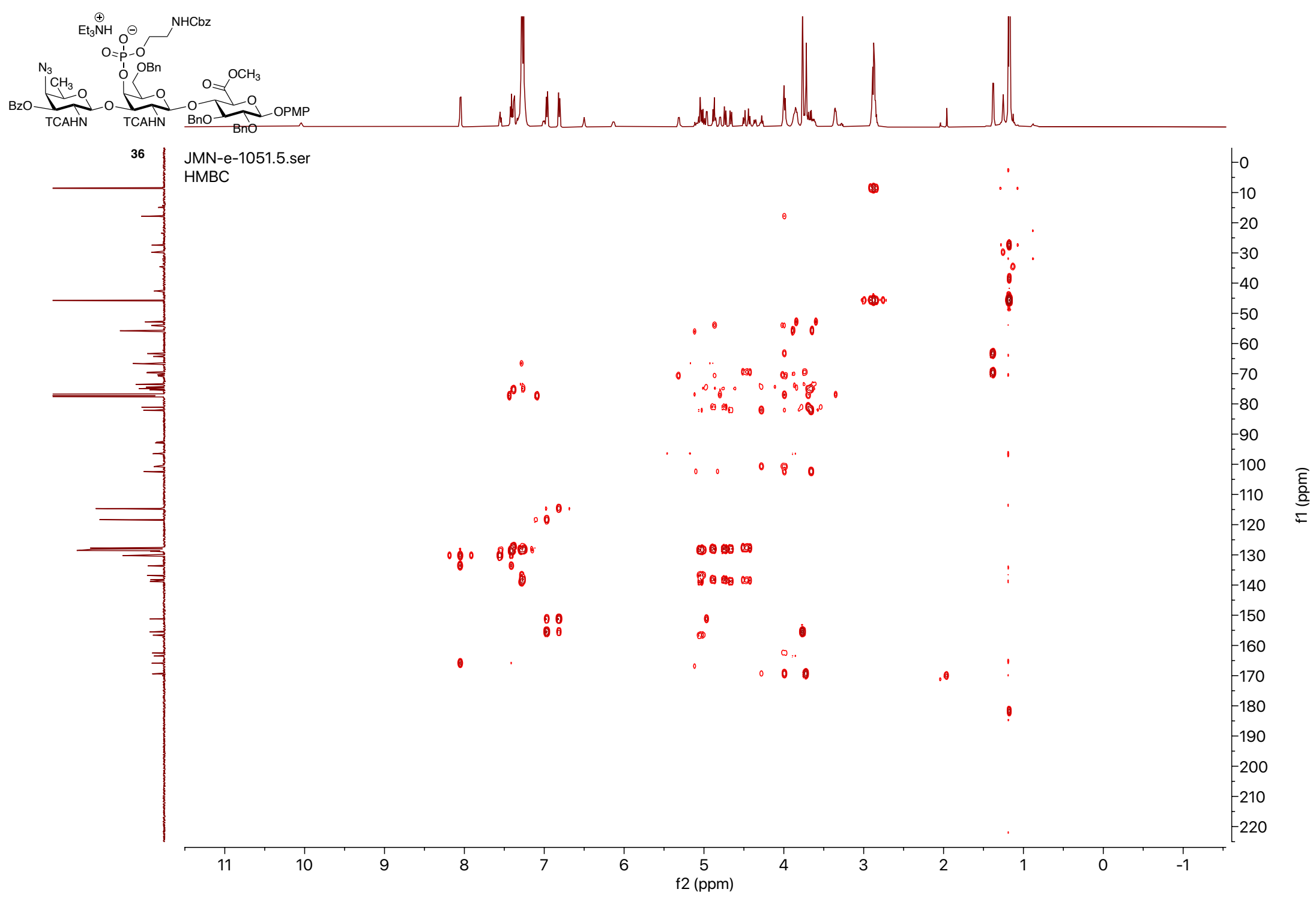


${ }^{31} \mathrm{P}$ NMR (162 MHz, $\left.\mathrm{CDCl}_{3}\right)$ of Compound 36

JMN-e-1051.1.fid

$\left.\mathrm{Et}_{3} \stackrel{\oplus}{\mathrm{NH}} \mathrm{O}^{\ominus}\right]^{\mathrm{NHCbz}}$

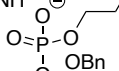

BZO $\underbrace{\mathrm{CH}_{3}}_{\text {TCAHN }}$

36

药

20000

$-19000$

$-18000$

$-17000$

$-16000$

$-15000$

$-14000$

$-13000$

$-12000$

$-11000$

$-10000$

9000

$-8000$

$-7000$

$-6000$

$-5000$

$-4000$

$-3000$

$-2000$

$-1000$

$-1000$

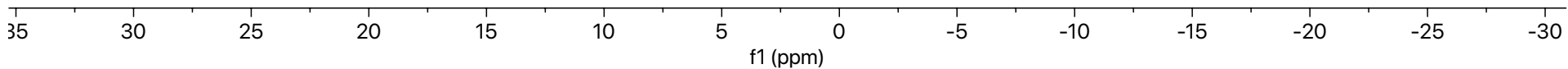

$-2000$ 
${ }^{1} \mathrm{H}$ NMR (600 MHz, $\left.\mathrm{D}_{2} \mathrm{O}\right)$ of Compound 29

JMN-e-1052.18.fid.

$1 \mathrm{H}$ Final Pdt

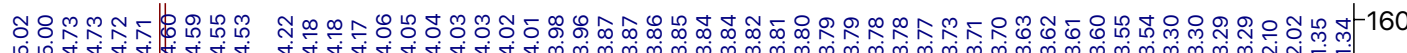
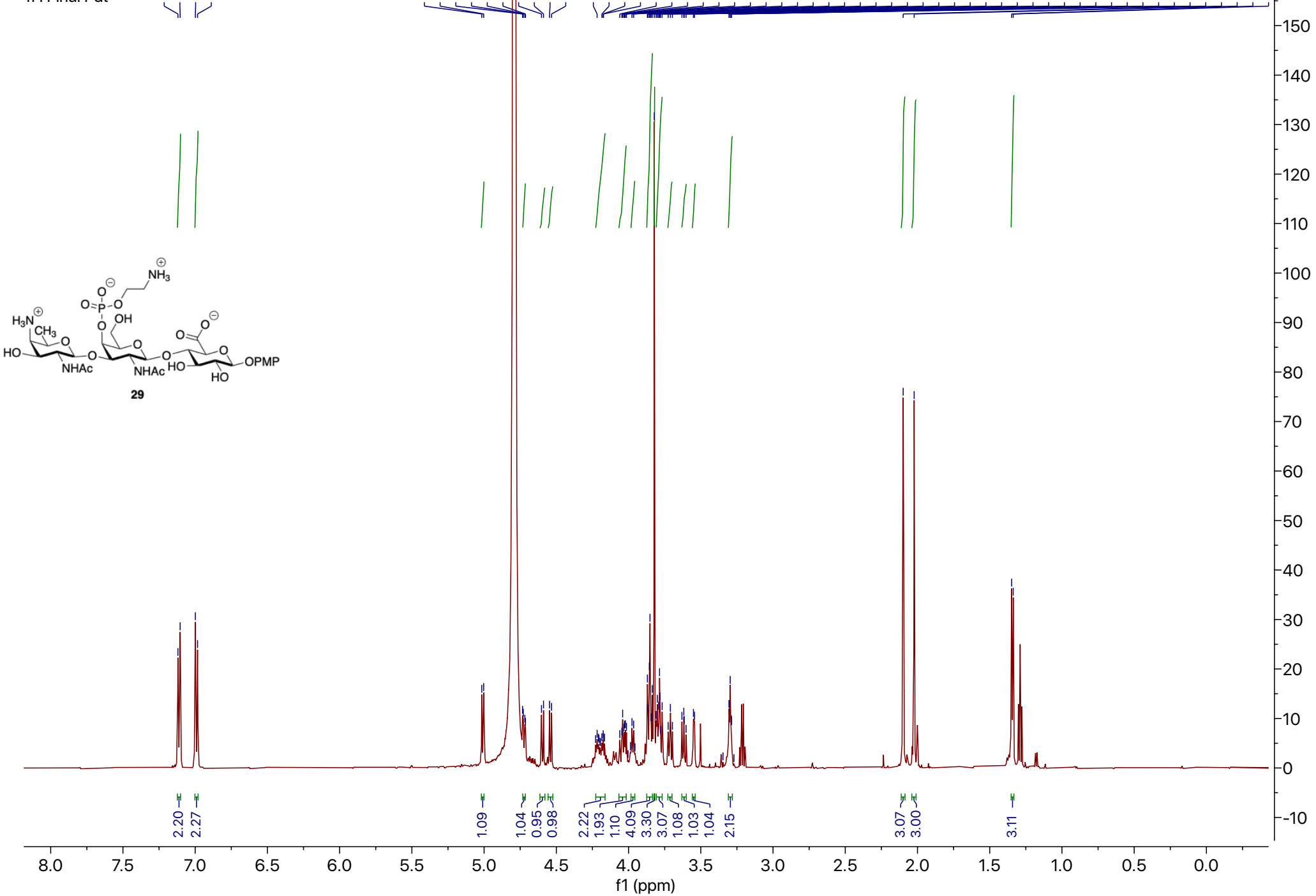
${ }^{13} \mathrm{C}$ NMR (151 MHz, $\left.\mathrm{D}_{2} \mathrm{O}\right)$ of Compound 29

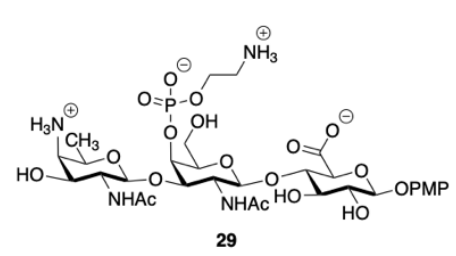

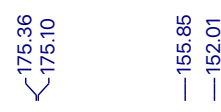

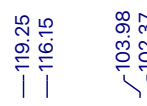

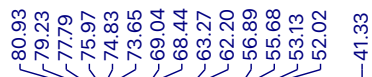

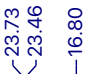

8000

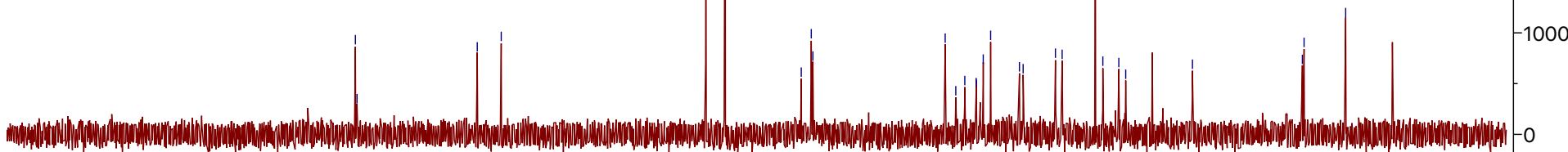


${ }^{1} \mathrm{H}-{ }^{13} \mathrm{C}$ HSQC $\left(\mathrm{D}_{2} \mathrm{O}\right)$ of Compound 29

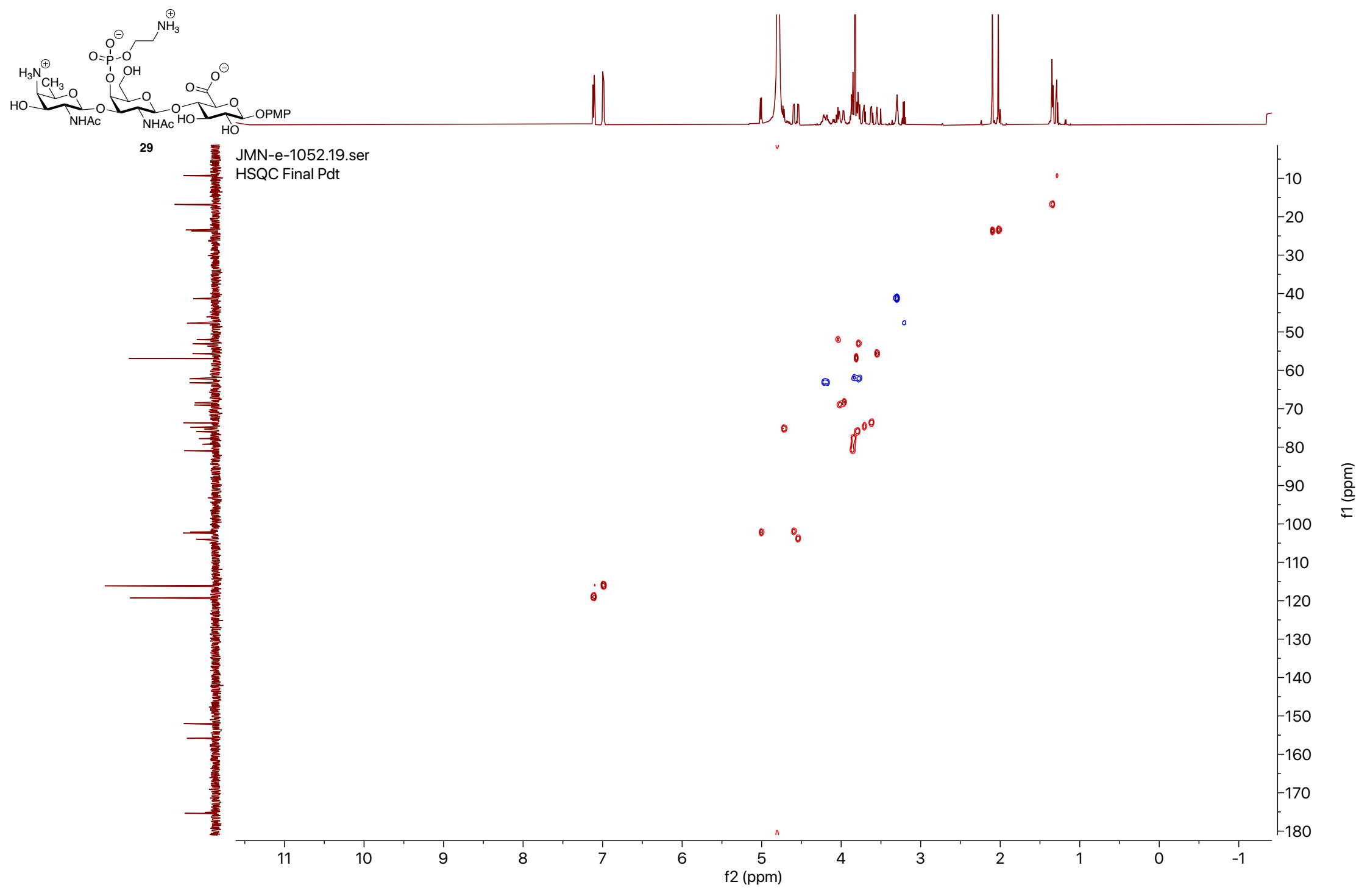


${ }^{1} \mathrm{H}-{ }^{1} \mathrm{H} \mathrm{COSY}\left(\mathrm{D}_{2} \mathrm{O}\right)$ of Compound 29

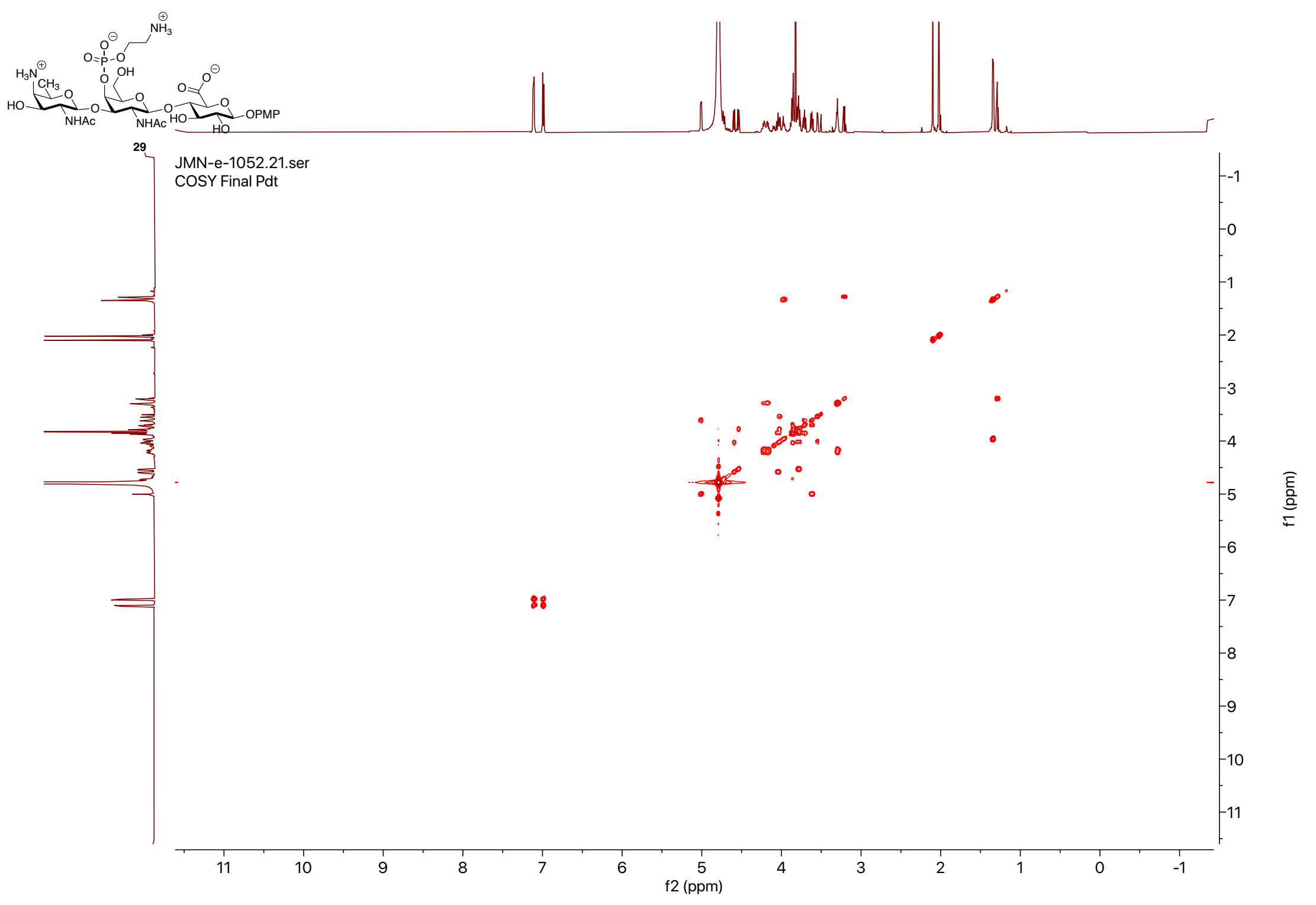


${ }^{1} \mathrm{H}-{ }^{13} \mathrm{C}$ HMBC $\left(\mathrm{D}_{2} \mathrm{O}\right)$ of Compound 29

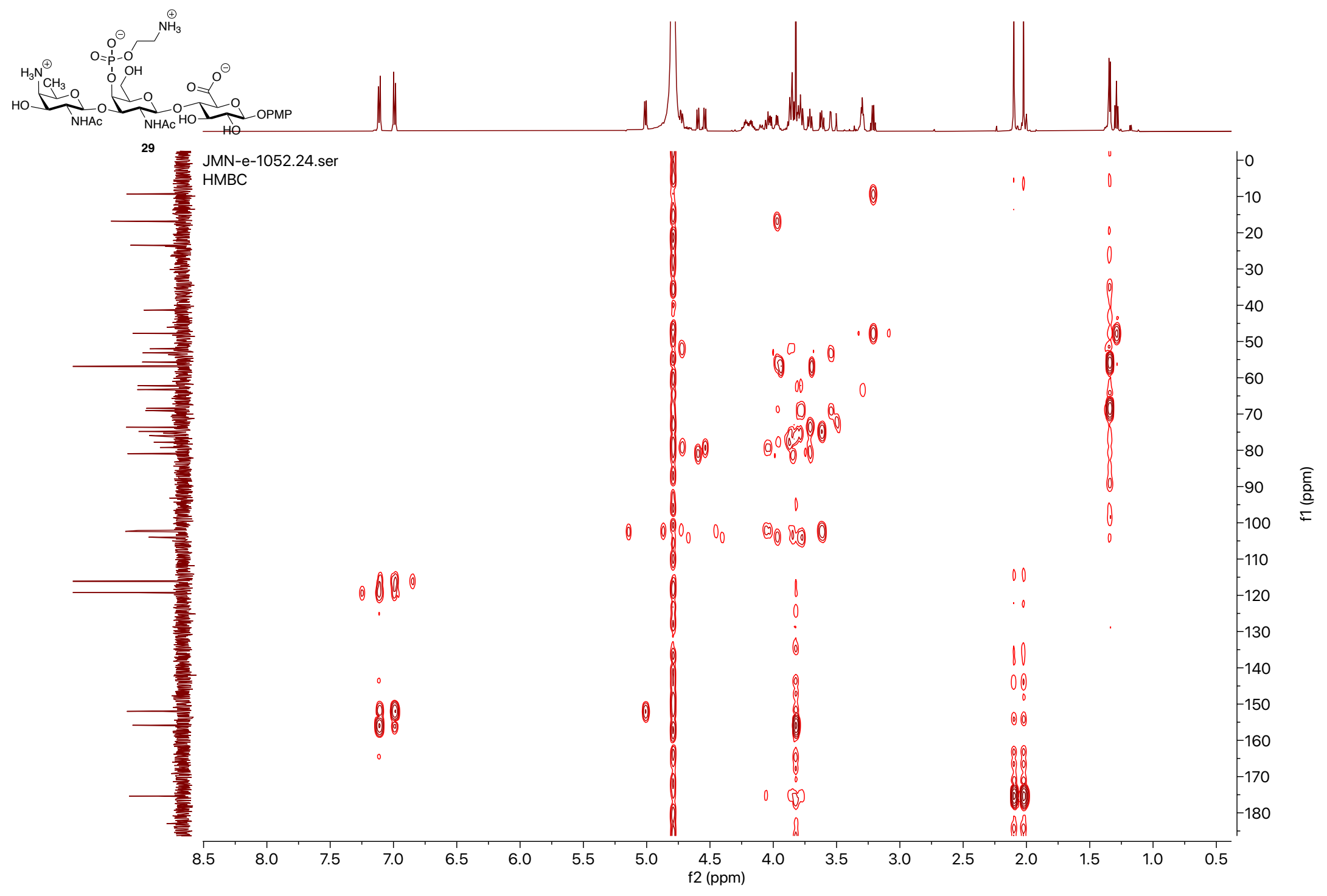


${ }^{31}$ P NMR (162 MHz, $\left.D_{2} O\right)$ of Compound 29
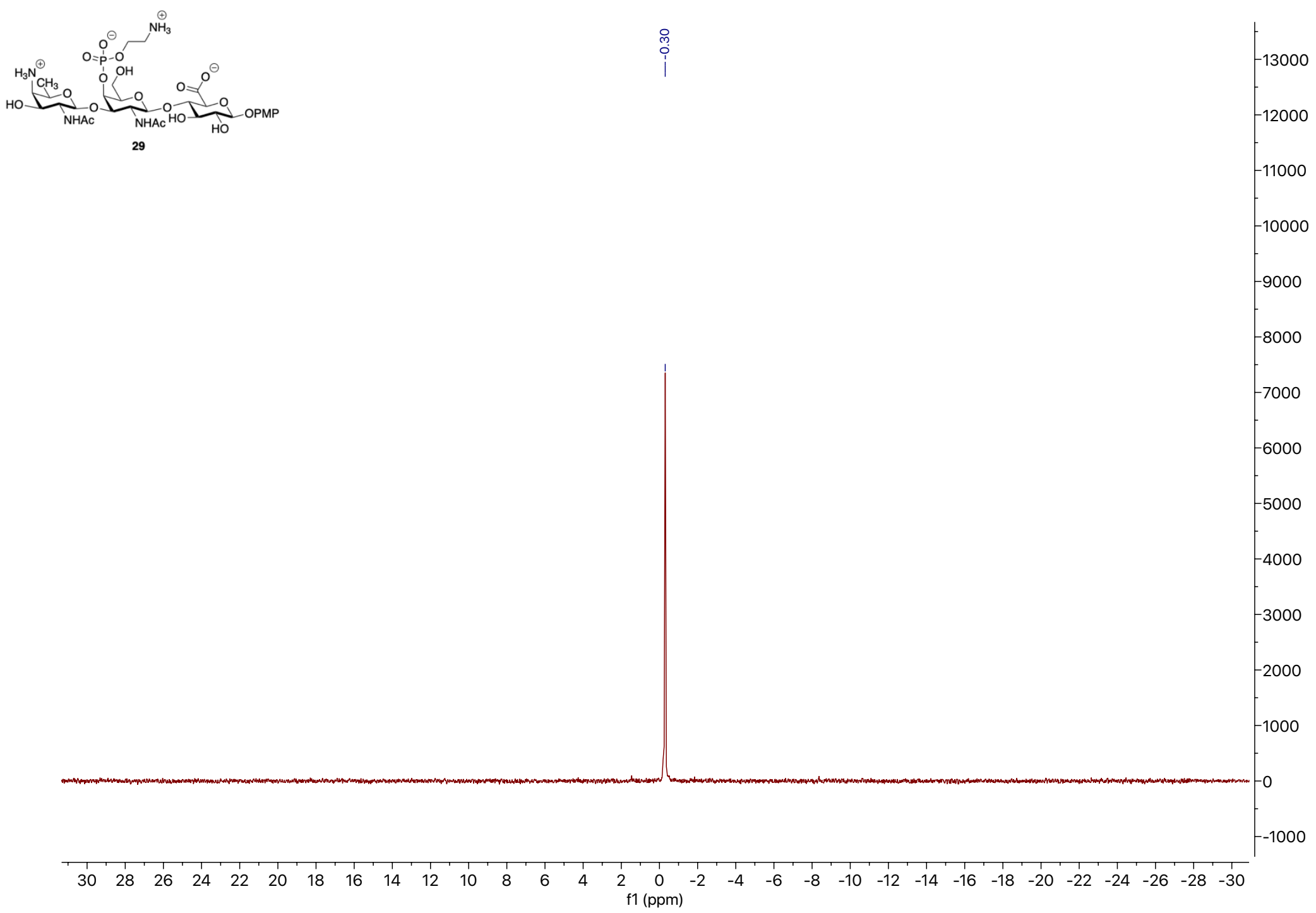


\section{References:}

1. Still, W. C.; Kahn, M.; Mitra, A. J. Org. Chem. 1978, 43 (14), 2923-2925.

2. Medgyes, A.; Farkas, E.; Liptak, A.; Pozsgay, V., Tetrahedron 1997, 53, 4159.

3. Gu, G.; Adabala, P. J. P.; Szczepina, M. G.; Borrelli, S.; Pinto, B. M. J. Org. Chem. 2013, 78, 8004-8019.

4. Verna, P.; Raj, R.; Roy, B.; Mukhopadhyay, B. Tetrahedron: Asymmetry. 2010, 21, 19, 2413-2418.

5. He, Q.Q.; Trim, P.J.; Snel, M.F.; Hopwood, J.J.; Ferro, V. Org. Biomol. Chem. 2018,16, 8791-8803.

6. Belot, F.; Jacquinet, J.C.; Carbohydrate Res. 2000, 325, 2, 93-106.

7. Keith, D. J. K; Townsend, S.D. J. Am. Chem. Soc. 2019, 141, 12939-12945.

8. Kwon, Y.U.; Liu, X.; Seeberger, P.H. Chem. Commun. 2005, 2280-2282.

9. Murakata, C.; Ogawa, T. Tetrahedron Lett. 1991, 32, 671-674.

10. Podilapu, A. R.; Kulkarni, S. S. Org. Lett. 2017, 19, 5466-5469.

11. Kondakova, A. N.; Kirsheva, N. A.; Arbatsky, N.P.; Shaikhutdinova, R. Z.; Shashkov, A. S.; Ivanov, S. A.; Anisimov, A. P.; Knirel, Y. A. Carbohydrate Res. 2015, 1-4. 